NBSIR 87-3618

\title{
Computer Software for the Computation of the Scattered Field and the Optical Microscope Image of Line Objects Patterned in Thick Layers
}

D. Nyyssonen

CD Metrology, Inc.

Germantown, MD 20874

December 1987

Prepared for:

U.S. DEPARTMENT OF COMMERCE National Bureau of Standards Alatinmal Engineering Laboratory

$-Q C$

100 ir Manufacturing Engineering

.456 I Engineering Division

$87-3618$

1987

C. 2 
COMPUTER SOFTWARE FOR THE COMPUTATION OF THE SCATTERED FIELD

AND THE OPTICAL MICROSCOPE IMAGE OF LINE OBJECTS PATTERNED IN THICK

\section{LAYERS}

D. Nyyssonen

CD Metrology, Inc.

Germantown, MD 20874

December 1987

Prepared for:

U.S. DEPARTMENT OF COMMERCE

National Bureau of Standards

National Engineering Laboratory

Center for Manufacturing Engineering

Precision Engineering Division

Gaithersburg, MD 20899

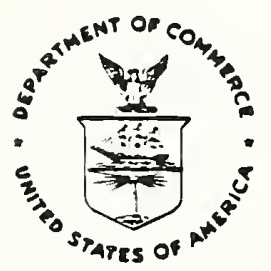

U.S. DEPARTMENT OF COMMERCE, C. William Verity, Secretary NATIONAL BUREAU OF STANDARDS, Ernest Ambler, Director 



\section{Table of Contents}

Page

Abstract . . . . . . . . . . . . . . . . 1 Introduction . . . . . . . . . . . . . . . 1 Software structure . . . . . . . . . . . . . 3 Implementation and Testing . . . . . . . . . . . 5 Accuracy . . . . . . . . . . . . . . . . . 6 Acknowlédgments . . . . . . . . . . . . . . 7 References . . . . . . . . . . . . . . . . 7 Table . . . . . . . . . . . . . . . . . . 9 Appendix I . . . . . . . . . . . . . . . . 10 Appendix II . . . . . . . . . . . . . . . 30 Appendix III . . . . . . . . . . . . . . . 52 

Computer Software for the computation of the scattered Field and the optical Microscope Image of Line Objects Patterned in Thick Layers

\section{Diana Nyyssonen \\ CD Metrology, Inc. \\ Germantown, MD 20874}

ABSTRACT: This report contains computer software for calculating optical microscope images of line objects patterned in thick layers $(>\lambda / 4$ thick). The algorithms used are based on a monochromatic, waveguide model which can predict the images of line objects with arbitrary edge geometry including multilayer structures with sloped, curved, asymmetric, and undercut edges. Along with the computer software listing, the mathematics of the model, a short description of its structure and use, and test cases for help in implementation are given.

KEY WORDS: computer software; diffraction; dimensional metrologY; linewidth; microscopy; optical imaging; optical metrology.

\section{INTRODUCTION}

The computer software described in this report was written in conjunction with the NBS project to develop fundamentally accurate optical measurement techniques for the width of micrometer and submicrometer lines patterned on integrated circuit wafers. Accurate and precise measurement techniques for linewidth are needed to improve yield, to ensure that lithographic and critical dimension (CD) measurement systems meet specification, to establish control of fabrication processes, and as input to device modeling and simulation programs.

In the course of research and development of the NBS laser linewidth measurement system [1], it was found that the microscope 
image profiles for lines patterned in thick layers ( $>\lambda / 4$ thick) were not properly predicted by the scalar theory conventionally used to calculate images of line objects $[2,3]$. Scalar imaging theory assumes that the object is planar (thin compared to the wavelength of the illumination) and can be represented by a complex transmittance or reflectance function. The scalar model does not take into account the multiple reflections or standing waves that may occur within the patterned layer or the edge effects that occur in thick layers.

A new model was developed by Nyyssonen [4, 5] based on a waveguide approach which characterizes the patterned layer by its complex dielectric constant and calculates the waveguide modes supported by the line structure. First, the mode expansion of the electromagnetic fields within the patterned layer is found and then the appropriate boundary conditions are used to calculate the scattered field.

Nyyssonen's model assumes that the line structure is patterned in a nonmagnetic layer which can be characterized by its complex index of refraction which is taken to be constant with depth within the layer. Thus, this model can be used to represent homogeneous line structures with vertical edge walls. The spatial function representing the variation in the dielectric constant (square of the complex index of refraction) in the layer is expanded in a Fourier series. This series is substituted into the wave equation and the eigenvalue solutions to this equation which represent the waveguide modes are found. Assuming a single incident plane wave normal to the surface, the boundary value problem at the layer interfaces is solved to determine the Fourier coefficients in the expansions for the transmitted and reflected fields. This method allows the use of conventional scalar imaging theory to compute the image when no polarization effects are present. In such a case, the E- and H-field components are equivalent and either may be used in the scalar imaging equations. 
To include the effects of partial coherence in the imaging system, it is possible to integrate over a finite illumination cone angle as long as the variation of the scattered field coefficients is negligible for the cone angle used. Hence, for layers approximately a few micrometers or less thick, finite coherence may be included.

This report covers the extension of this model by kirk and Nyyssonen [6] to line structures of arbitrary edge geometry whose index of refraction may vary with depth in the layer. This line structure is approximated by subdividing it into a set of sublayers, each consisting of a line object with vertical edges and having a constant index of refraction over its small interval of depth. Each sublayer is treated in the same way as the single layer of Nyyssonen's earlier model. The waveguide modes and solution for the electromagnetic fields are found for each sublayer. There are now $n+1$ boundary value equations where $n$ is the number of sublayers. These equations when solved allow for the substitution of a single "equivalent" planar scattering layer for the multilayer structure. Thereafter, the solution for the transmitted and reflected field components and image are found in the same manner as for the single layer case. This model therefore allows for the modeling of line structures which contain different materials, have curved edges, asymetry, and unpatterned (or patterned) sublayers. The mathematical details of the model are given in the expanded version of ref. [6] reproduced in Appendix III. A list of definitions of symbols used in the software and Appendix III is given in the attached table.

\section{Software Structure}

As input to the software, the line structure may be characterized either by giving the index of refraction, thickness, and edge locations for the individual sublayers, or by a single refractive index, total thickness, and the polynomial coefficients used to 
describe the edge geometry (See Appendix III.) In the latter case, the number of layers used to approximate this structure in the calculations must be designated. A good rule of thumb to use in determining the number of layers is to divide the change in linewidth (between the top and bottom interfaces) by $0.1 \mathrm{\mu m}$ and use a value larger than this. For most cases of interest in integrated circuit processing, 9 layers are sufficient. The program arrays, as written, allow up to 20 layers. The program calculates both the scattered field and the microscope image for TE-mode illumination. That is, the direction of polarization (i.e. E-field) is assumed to be parallel to the length of the line structure.

The scattered field is given in terms of the Fourier coefficients of the resulting far field diffraction pattern. In the test cases shown, 45 coefficients are given corresponding to \pm 22 diffracted orders at diffraction angles corresponding to $n \lambda / P$ where $\mathrm{n}$ is the order number, $\lambda$ the wavelength, and $\mathrm{P}$ the period. The line structure is assumed to be repeated at a period $\mathrm{P}$ for convenience in calculating the Fourier series coefficients. An isolated line image is calculated by taking $P$ large enough so that there is no influence from adjacent edges. Near resonances, this is not always possible due to time or storage limits on array sizes. The largest period used has been $12 \mu \mathrm{m}$ corresponding to a $90 \times 90$ complex matrix for the boundary condition equations.

The microscopic image is calculated using conventional scalar imaging theory with the complex Fourier coefficients of the scattered field substituted for those of the planar object traditionally used. The software, as written, assumes a 1-D imaging system with a finite illumination cone angle. The Fourier coefficients of the scattered field are assumed to be constant over this angle. The calculations are accurate for a 2-D system as long as the condenser numerical aperture (N.A.) is much smaller 
than that of the imaging objective. Hence, the calculations will accurately predict the image waveforms for the NBS laser system which has an objective N.A. of 0.85 or 0.95 and an illumination aperture $1 / 5$ that of the objective at a wavelength of $514 \mathrm{~nm}$.

The software also allows the calculation of the image for varying focus positions. Best focus is the top surface of the patterned layer. For all other focus positions, a quadratic phase factor is introduced (just as in scalar image theory).

The input for focus position is given as the number of wavelengths of defocus $m$ where $m$ is a dimensionless quantity. The relationship between the displacement of the measurement plane from the Gaussian focal plane, $z$, is given by

$$
z=\frac{2}{\tan ^{2} \theta} m
$$

where $\lambda$ is the wavelength and $\tan \theta$ is substituted for N.A. (=sin $\theta$ ) for the high numerical apertures conventionally used for linewidth measurement.

\section{Implementation and Testing}

This software (See Appendix I) is written in ANSI FORTRAN 77 consisting of a main program and 9 modules. The software is portable except for the complex matrix subroutines used for calculation of the eigenvectors and eigenvalues and matrix inversions. The software requires highly accurate routines because of the cascading of matrix multiplications and inversions. Those used at NBS on the Cyber $855^{*}$ are from the NAG library ${ }^{*}$ [7]. If these

\footnotetext{
*Certain commercial equipment, instruments, or materials are identified in this paper in order to adequately specify experimental or computational procedure. Such identification does not imply recommendation or endorsement by the National Bureau of standards, nor does it imply that the materials or equipment identified are necessarily the best available for the purpose.
} 
routines are unavailable, the user may substitute others as noted in the software. However, several test conditions should be met. A symmetric line object should produce symmetric Fourier coefficients to at least six significant figures. Also, a single-layer, thick line object with complex index of refraction should produce the same image structure as for a single layer when divided into 20 sublayers (or the maximum number of sublayers to be used). Repeatability to three or four significant figures in the image is sufficient to ensure this. If the matrix routines are inaccurate, the errors will increase as the number of layers is increased. Test cases are given in Appendix II. These six test cases are designed to test different portions of the software. successful execution of one test case does not guarantee accuracy for the others.

All of the data calculated are output to files for use with available graphics. Plotting routines are not included here because they tend to be system dependent and user requirements may vary.

\section{Accuracy.}

Testing of this software poses a challenging problem in that the only cases for which answers are known do not fully test the algorithms. For example, in comparing this model with scalar theory, agreement can be expected only in the limit as the thickness of the patterned layer approaches zero. Test case \#2 for a patterned 0.09 um thick chromium layer is one such case which has been shown to agree with scalar theory. (See ref. 5.) Comparison has also been done for patterned thin layers of silicon dioxide showing excellent agreement. (See ref. 4.) In addition, it is required that the ratio of reflectivities of the patterned layer and substrate (or sublayers) away from the edges agrees with the values calculated from the Fresnel equations. (See ref. 8.) Otherwise, calculations can at this time only be compared with experimental measurements. This has been done for some cases 
$[6,9]$. However, although the agreement is good in most cases, the accuracy is suspect near resonances (where the line dimensions of width and/or thickness are equal to an integer multiple of the wavelength) and for small line dimensions. In both cases, the energy in the high spatial frequencies increases and errors in the eigenvalues and eigenvectors are expected to increase due to truncation errors.

\section{Acknowledgments}

A number of people have contributed to this software development. The original software was written for the Univac 1108 computer by Chris Kirk while a research associate at NBS. The author wishes to especially thank Ruth Varner, NBS, for her assistance in converting this software for use on the Cyber 855 . $^{\text {* The pre- }}$ sent software has been modified by the author to work for patterned metal layers. Other modifications to simplify its use and provide additional documentation have also been made.

\section{References}

1. D. Nyyssonen, "Calibration of Optical Systems for Linewidth Measurements on Wafers," Opt. Eng. 21, 882-887 (1982).

2. I. C. Martin, The Theory of the Microscope, (Blackie, London, 1966), Chapters V and VIII.

3. E. C. Kintner, "Method for the Calculation of Partially Coherent Imagery," Appl. Opt. 17, 2747-2753 (1978).

4. D. Nyyssonen, "Theory of Optical Edge Detection and Imaging of Thick Layers," J. Opt. Soc. Am. 2, 1425-1436 (1982).

*See previous footnote. 
5. D. Nyyssonen, "Optical Linewidth Measurement on Patterned Metal Layers," Proc. SPIE Vol. 480, Integrated Circuit Metrology II, 65-70 (1984).

6. D. Nyyssonen and C. Kirk, "Modeling the Optical Microscope Images of Thick Layers for the Purpose of Iinewidth Measurement," Proc. SPIE Vol. 538, Optical Microlithography IV, $179-187$ (1985).

7. These routines are available from: Numerical Algorithms Group (NAG) Ltd., 7 Banbury Road, Oxford, Oxfordshire ox2 $6 \mathrm{NN}$, England.

8. D. Nyyssonen, "Narrow-Angle Laser Scanning Microscope System for Linewidth Measurement on Wafers," NBSIR ( to be published).

9. C. Rirk, "A Study of the Instrumental Errors in Linewidth and Registration Measurements Made with an Optical Microscope," Presented at the SPIE 1987 Santa Clara Symposium on Microlithography, Vol. 775, Integrated Circuit Metrology, Inspection and Process Control. 
Table: Definition of Symbols Used in Software

\begin{tabular}{cc} 
Math & $\begin{array}{c}\text { Software } \\
\text { Symbol }\end{array}$ \\
\hline
\end{tabular}

Free space wavelength in microns and 3 rd order

The $X$ position offset for each layer

The $z$ locations of the layer interfaces

Spatial frequency of the grating

Free space wavenumber (in units of $\mu \mathrm{m}^{-1}$ )

Fourier coefficients of dielectric constant

Eigenvalue matrix

Eigenvalues of the layers

Eigenvectors of the layers

Boundary equation matrices $\lambda$

T

N

P

$\hat{n}_{0}$

$\hat{n}_{s}$

$\hat{n}_{n}$

$x_{j}$

$\mathrm{z}_{j}$

WAVE

TI

NS

PER

CRI (0)

CRI ( 21)

CRI (N)

$W(J)$

$\mathrm{PO}(\mathrm{J})$

$\mathrm{XP}(\mathrm{J})$

$2 P(N)$

$\mathrm{z}_{\mathrm{n}}$

$1 / P$

$\mathrm{k}_{0}$

SPAFRE

RKO

$E_{q, n}$

EQ ( I )

$D_{i, j}$

$D(I, J)$

$a_{m, n}$

$\operatorname{VAL}(\mathrm{M})$

$B_{j, m, n}$

VEC
(A) coefficients

Fourier coefficients of the pseudoobject

1-D image of the line
$A_{m, 1}, A_{m, 1}^{\prime} \quad A V(M)$

$F C(J)$

$\operatorname{ELEC}(I)$ 
Appendix I

Computer software Listing (THKIMAG) 
$2 \mathrm{C}$

$3 \mathrm{C}$

$4 \mathrm{C}$

$5 \mathrm{C}$

$6 \mathrm{C}$

$7 \mathrm{C}$

$8 \mathrm{C}$

$9 \mathrm{C}$

$10 \mathrm{C}$

$11 \mathrm{C}$

$12 \mathrm{C}$

$13 \mathrm{C}$

$14 \mathrm{C}$

$15 \mathrm{C}$

$16 \mathrm{C}$

$17 \mathrm{C}$

$18 \mathrm{C}$

$19 \mathrm{C}$

$20 \mathrm{C}$

$21 \mathrm{C}$

$22 \mathrm{C}$

$23 \mathrm{C}$

$24 \cdot C$

$25 \mathrm{C}$

$26 \mathrm{C}$

$27 \mathrm{C}$

$28 \mathrm{C}$

$29 \mathrm{C}$

$30 \mathrm{C}$

$31 \mathrm{C}$

$32 \mathrm{C}$

$33 \mathrm{C}$

$34 \mathrm{C}$

$35 \mathrm{C}$

$36 \mathrm{C}$

$37 \mathrm{C}$

$38 \mathrm{C}$

$39 \mathrm{C}$

$40 \mathrm{C}$

$41 \mathrm{C}$

$42 \mathrm{C}$

$43 \mathrm{C}$

$44 \mathrm{C}$

$45 \mathrm{C}$

$46 \mathrm{C}$

$47 \mathrm{C}$

$48 \mathrm{C}$

$49 \mathrm{C}$

$50 \mathrm{C}$

PROGRAM THKIMAG

THE ORIGINAL VERSION OF THIS PROGRAM WAS WRITTEN BY CHRIS KIRK, WHILE AN EMPLOYEE OF VICKERS INSTRUMENTS, (HAXBY ROAD, YORK, NORTH YORKSHIRE, ENGLAND) WHILE A RESEARCH ASSOCIATE AT THE NATIONAL BUREAU OF STANDARDS, GAITHERSBURG, MARYLAND, USA. SEPTEMBER 1984.

THE CURRENT VERSION HAS BEEN MODIFIED BY D. NYYSSONEN TO WORK FOR METAL LAYERS. JUNE 1986

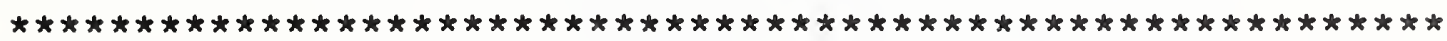

THIS PROGRAM COMPUTES THE OPTICAL IMAGE OF LINE OBJECTS WITH ARBITRARY EDGE GEOMETRY, RATTERNED IN THICK LAYERS INCLUDING MULTILAYER STRUCTURES WITH SLOPING, CURVED, AND UNDERCUT EDGES, AS WELI AS ASYMMETRIC OBJECTS.

SEE REFERENCE: "MODELING OF THE OPTICAL IMAGING OF LINES PATTERNED IN THICK LAYERS WITH VARIABLE EDGE GEOMETRY," BY D. NYYSSONEN AND C. P. KIRK

ALI EQUATION NUMBERS GIVEN REFER TO THIS MANUSCRIPT FOR QUESTIONS CONCERNING THIS PROGRAM CONTACT:

R. D. LARRABEE, PRECISION ENGINEERING DIVISION, NATIONAL BUREAU OF STANDARDS OR D. NYYSSONEN, CD METROLOGY, INC.

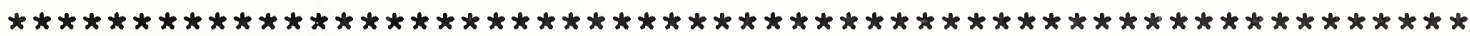

SYSTEM DEPENDENT FEATURES.

ALTHOUGH THIS SOFTWARE HAS BEEN WRITTEN IN FORTRAN 77 IN ORDER TO ALLOW FOR EASY PORTABILITY, THERE ARE A NUMBER OF FEATURES WHICH MAY BE SYSTEM DEPENDENT.

1. A FORTRAN 77 COMPILER IS REQUIRED.

2. THE SYSTEM MUST SUPPORT CERTAIN NAG LIBRARY ROUTINES.

A NOTE CONCERNING THE NAG IIBRARY.

THIS PROGRAM USES THE FOLLOWING NAG ROUTINES.

FO2AKF FO4ADF

THESE ARE SINGLE PRECISION VERSIONS OF IIBRARY ROUTINES FOR THE CYBER WHICH MAY NOT BE SUPPORTED ON ALL MACHINES.

THESE ROUTINES ARE AVAILABLE FROM: 
NUMERICAL ALGORITHMS GROUP (NAG) LTD, 7 BANBURY ROAD, OXFORD, OXFORDSHIRE. OX2 6NN. ENGLAND.

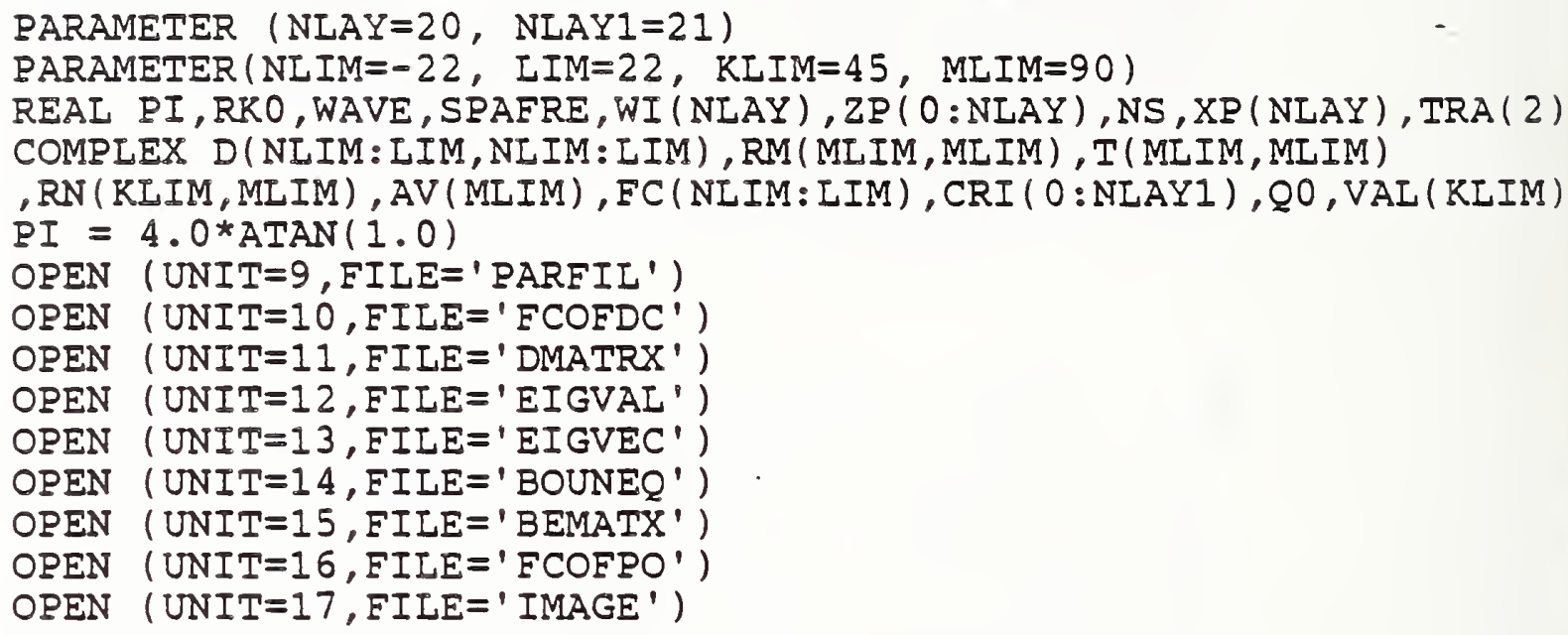

SET UP THE PARAMETERS FOR THE SECTIONED IINE.

INPUT

WAVE = FREE SPACE WAVELENGTH IN MICRONS.

NS $=$ THE NUMBER OF LAYERS.

PER $=$ THE PERIOD.

CRI $(0)=$ RI OF THE AIR LAYER. 
$101 \mathrm{C}$

$102 \mathrm{C}$

$103 \mathrm{C}$

$104 \mathrm{C}$

$105 \mathrm{C}$

$106 \mathrm{C}$

$107 \mathrm{C}$

$108 \mathrm{C}$

$109 \mathrm{C}$

$110 \mathrm{C}$

$111 \mathrm{C}$

$112 \mathrm{C}$

$113 \mathrm{C}$

$114 \mathrm{C}$

$115 \mathrm{C}$

$116 \mathrm{C}$

$117 \mathrm{C}$

$118 \mathrm{C}$

$119 \mathrm{C}$

$120 \mathrm{C}$

$121 \mathrm{C}$

$122 \mathrm{C}$

$123 \mathrm{C}$

$124 \mathrm{C}$

$125 \mathrm{C}$

$126 \mathrm{C}$

$127 \mathrm{C}$

$128 \mathrm{C}$

$129 \mathrm{C}$

$130 \mathrm{C}$

$131 \mathrm{C}$

$132 \mathrm{C}$

$133 \mathrm{C}$

$134 \mathrm{C}$

$135 \mathrm{C}$

$136 \mathrm{C}$

$137 \mathrm{C}$

$138 \mathrm{C}$

$139 \mathrm{C}$

140

141

$142 \mathrm{C}$

$143 \mathrm{C}$

$144 \mathrm{C}$

$145 \mathrm{C}$

$146 \mathrm{C}$

$147 \mathrm{C}$

$148 \mathrm{C}$

$149 \mathrm{C}$

$150 \mathrm{C}$

CRI $(21)=$ RI OF THE SUBSTRATE.

ID = DEFINE LINE: PARAMETRICALLY $(0)$

$=\quad:$ LAYER BY LAYER(1).

$W(I)=W I D T H$ POLYNOMIAL COEFFICIENTS. I=ORDER

PO(I) = STARTING POINTS FOR 1ST, 2ND AND 3RD ORDER.

CRI (1) = REFRACTIVE INDEX OF PATTERNED LAYER.

TL = LAYER THICKNESS.

TRA $(1)=$ PHOTOMULTIPLIER SLIT WIDTH.

TRA $(2)=$ TELEVISION CAMERA GAUSSIAN WIDTH PARAMETER.

LSKIP $=0$ : GENERATE A NEW FOURIER SERIES FOR THE PSEUDO OBJECT.

1: USE PREVIOUSLY GENERATED FOURIER SERIES. -1: GENERATE THE IMAGE.

$D F=$ DEFOCUS IN WAVES WHERE DF IS A DIMENSIONLESS QUANTITY. IF DEFOCUS IS KNOWN IN TERMS OF DISPLACEMENT OF THE MEASUREMENT PLANE FROM THE FOCAL PLANE DZ, IT MUST BE CONVERTED TO WAVES USING THE RELATIONSHIP: $D Z=(2 *$ WAVE $/($ TAN $($ ANGLE $)) * * 2) * D F$

WHERE ANGLE IS THE SAME AS DEFINED BY N.A. = SIN(ANGLE) INPUT IF ID $=1$.

WI $(N)=$ THE WIDTH OF THE LINES IN THE NTH LAYER.

$Z P(N)=$ THE $Z$ LOCATIONS OF THE LAYER INTERFACES.

$\mathrm{CRI}(\mathrm{N})=\mathrm{RI}$ OF EACH LAYER.

$X P(N)=$ THE X POSITION OFFSET FOR EACH LAYER.

COMPUTATION

SPAFRE = SPATIAL FREQUENCY OF THE GRATING (1/PERIOD).

COMPUTATIONS IF ID $=0$.

CRI(N) = RI OF EACH LAYER.

WI(N) = THE WIDTHS OF THE LINES IN EACH LAYER.

$Z P(N)=$ THE $Z$ LOCATIONS OF THE LAYER INTERFACES. THE TOP SURFACE IS ASSUMED TO BE $\mathrm{z}=0$.

$X P(N)=$ THE $X$ POSITION OFFSET FOR EACH LAYER (FOR ASYMMETRIC CROSS-SECTIONS) .

COMPUTATION

RKO = FREE SPACE WAVENUMBER (IN UNITS OF 1/UM).

CALL LINE ( $P I$, WAVE, RKO , CRI, ZP,WI, NS , SPAFRE,

TRA, XP, DF , LSKIP)

NOTE: CALCULATIONS ARE FOR LINE OBJECT, SPACES ARE CALCULATED BY TAKING LINEWIDTH = PERIOD MINUS DESIRED SPACE WIDTH.

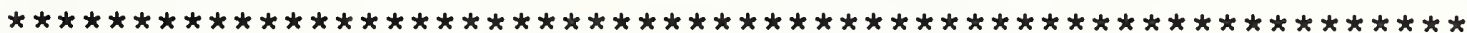

IF LSKIP $=1$ THEN GO STRAIGHT TO THE IMAGE SUBROUTINE. 
151

$152 \mathrm{C}$

$153 \mathrm{C}$

$154 \mathrm{C}$

$155 \mathrm{C}$

$156 \mathrm{C}$

$157 \mathrm{C}$

$158 \mathrm{C}$

$159 \mathrm{C}$

$160 \mathrm{C}$

$161 \mathrm{C}$

$162 \mathrm{C}$

$163 \mathrm{C}$

$164 \mathrm{C}$

$165 \mathrm{C}$

$166 \mathrm{C}$

$167 \mathrm{C}$

$168 \mathrm{C}$

169

170

171

17210

$173 \mathrm{C}$

$174 \mathrm{C}$

$175 \mathrm{C}$

$176 \mathrm{C}$

$177 \mathrm{C}$

$178 \mathrm{C}$

$179 \mathrm{C}$

$180 \mathrm{C}$

$181 \mathrm{C}$

182

$183 \mathrm{C}$

$184 \mathrm{C}$

$185 \mathrm{C}$

$186 \mathrm{C}$

$187 \mathrm{C}$

$188 \mathrm{C}$

$189 \mathrm{C}$

$190 \mathrm{C}$

$191 \mathrm{C}$

$192 \mathrm{C}$

$193 \mathrm{C}$

$194 \mathrm{C}$

195

196

197

198

199

200

IF (LSKIP.EQ.1) GO TO 20

FIND THE EIGENVALUES AND EIGENVECTORS FOR EACH LAYER.

D IS THE EIGENVALUE MATRIX FOR EACH LAYER. THE EIGENVALUES AND EIGENVECTORS ARE FOUND FOR EACH LAYER AND THEN STORED ON DISK, STARTING WITH THE TOP LAYER.

THE DIFFRACTION SERIES IS TRUNCATED FOR DIFFRACTION ANGLES WHICH EXCEED 90 DEGREES IN AIR. THIS IS SET BY THE VARIABLE 'IIM'. IIM AND ITS RELATED VARIABLES ARE SET IN THE PARAMETER STATEMENTS.

$I=$ (REFRACTIVE INDEX OF AIR)* (PERIOD)/(WAVELENGTH)

NLIM $=-I, \operatorname{LIM}=I, \operatorname{KLIM}=2 L+1, \operatorname{MLIM}=2(2 L+1)$

DO $10 \mathrm{~N}=1$, NS

CAIL SETUPD ( PI, WI (N) , CRI, D, SPAFRE, $N, X P(N)$, WAVE, $Q 0, E Q 2$ )

CALI EIGD ( D, N, QO, RKO, WAVE, SPAFRE, EQ2)

CONTINUE

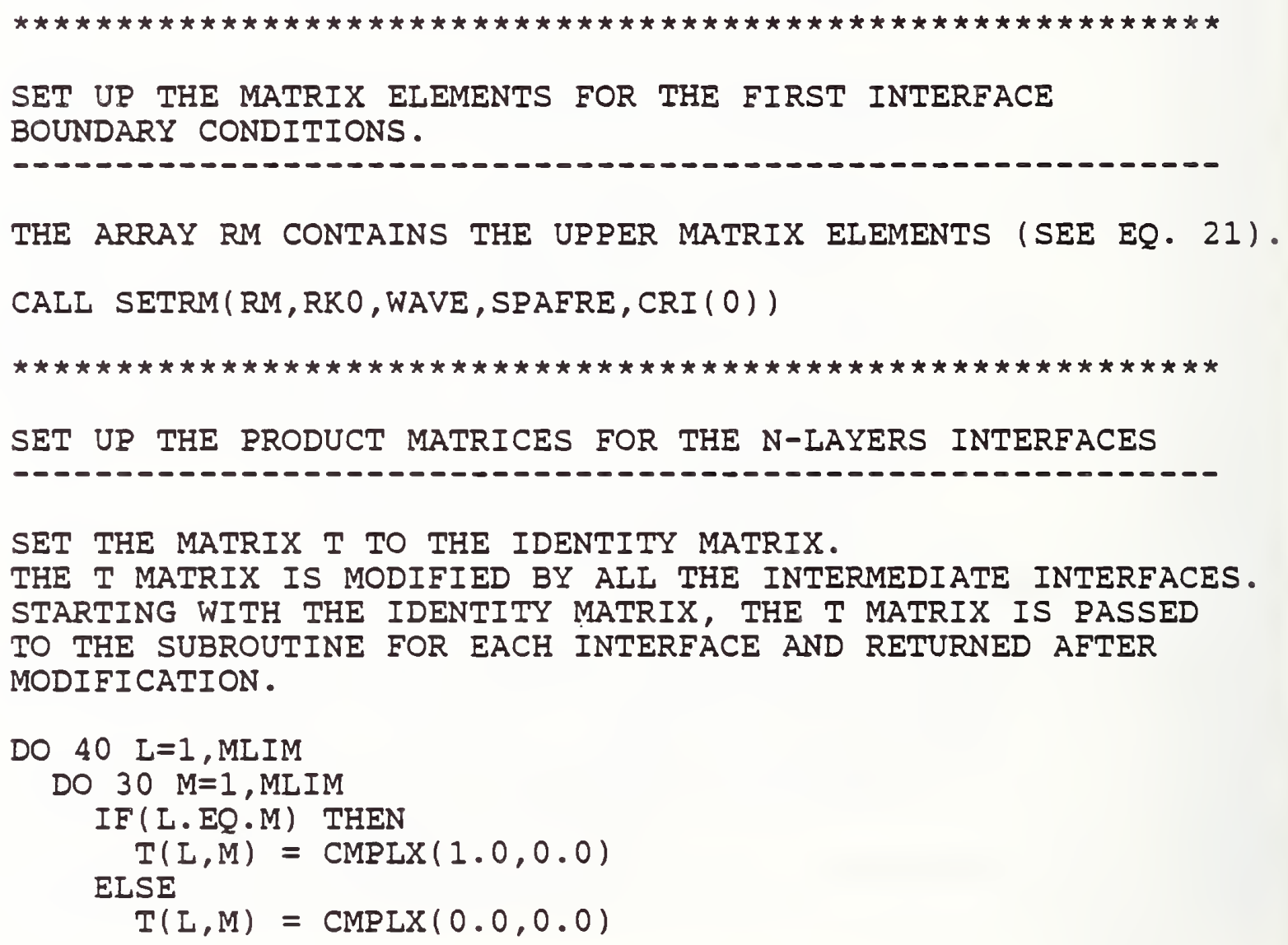

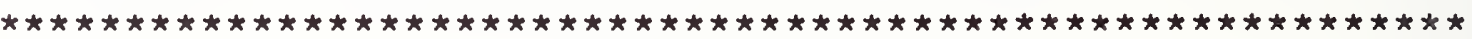

SET UP THE MATRIX ELEMENTS FOR THE FIRST INTERFACE BOUNDARY CONDITIONS.

THE ARRAY RM CONTAINS THE UPPER MATRIX ELEMENTS (SEE EQ. 21 ).

CALL SETRM(RM, RKO, WAVE, SPAFRE, CRI (0))

SET UP THE PRODUCT MATRICES FOR THE N-LAYERS INTERFACES

SET THE MATRIX T TO THE IDENTITY MATRIX.

THE T MATRIX IS MODIFIED BY ALI THE INTERMEDIATE INTEREACES. STARTING WITH THE IDENTITY MATRIX, THE T MATRIX IS PASSED TO THE SUBROUTINE FOR EACH INTERFACE AND RETURNED AFTER MODIFICATION.

DO 40 I $=1$, MLIM

DO $30 \mathrm{M}=1$, MLIM

IF ( I.EQ.M) THEN

$T(L, M)=\operatorname{CMPLX}(1.0,0.0)$

ELSE

$T(L, M)=\operatorname{CMPLX}(0.0,0.0)$ 


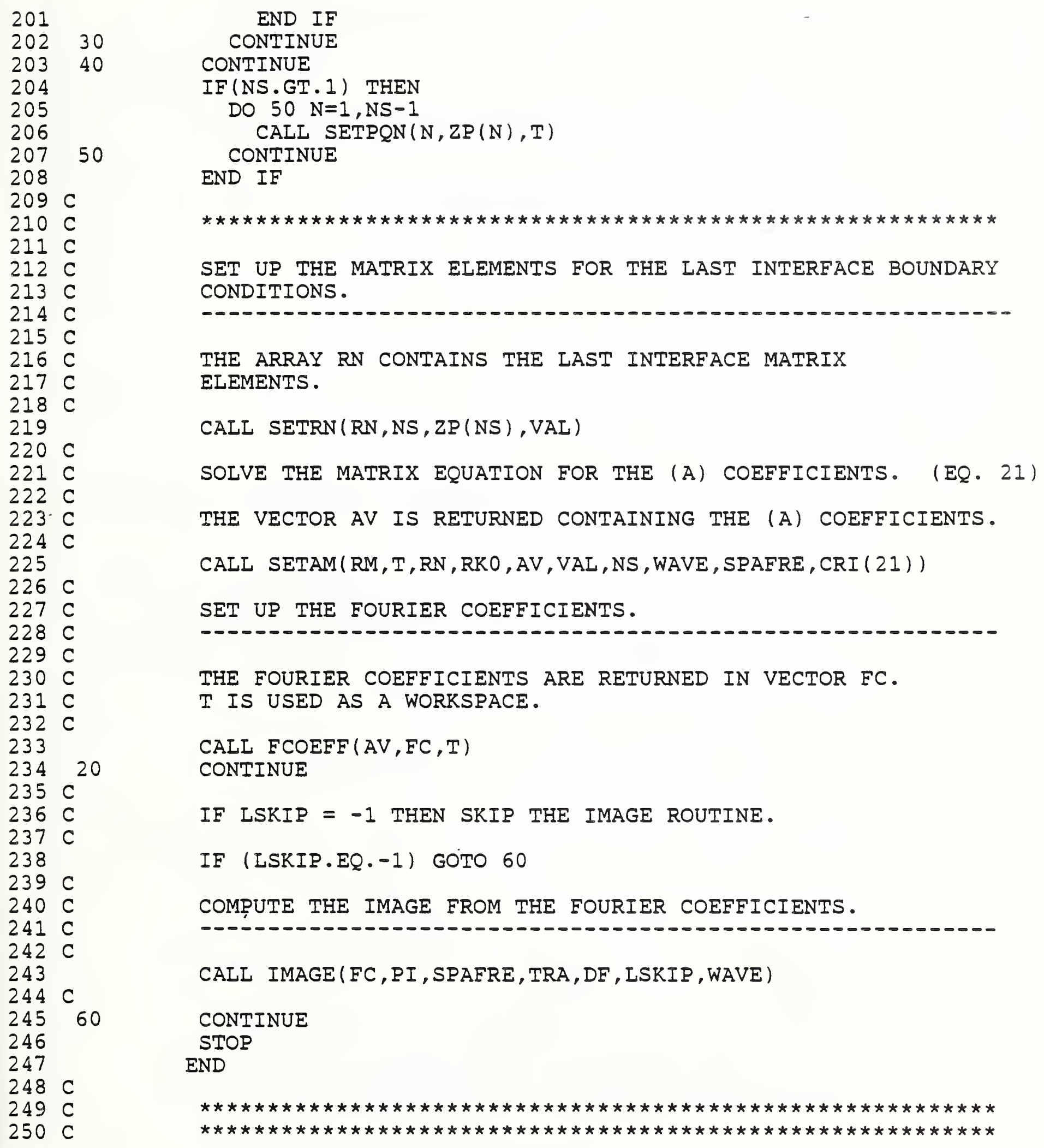

END IF CONTINUE CONTINUE IF (NS.GT.1) THEN DO $50 \mathrm{~N}=1, \mathrm{NS}-1$ CALL $\operatorname{SETPQN}(N, Z P(N), T)$ CONTINUE END IF

SET UP THE MATRIX ELEMENTS FOR THE LAST INTERFACE BOUNDARY CONDITIONS.

THE ARRAY RN CONTAINS THE LAST INTERFACE MATRIX
ELEMENTS.

CALL SETRN (RN, NS, ZP(NS), VAL)

SOLVE THE MATRIX EQUATION FOR THE (A) COEFFICIENTS. (EQ. 21) THE VECTOR AV IS RETURNED CONTAINING THE (A) COEFFICIENTS. CALI SETAM(RM, T,RN , RKO , AV, VAI, NS, WAVE, SPAFRE, CRI (21)) SET UP THE FOURIER COEFFICIENTS.

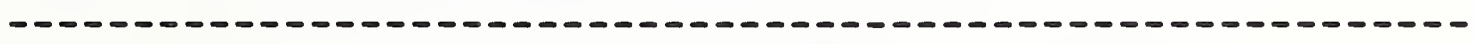
THE FOURIER COEFFICIENTS ARE RETURNED IN VECTOR FC. $T$ IS USED AS A WORKSPACE.

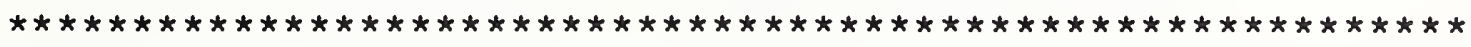

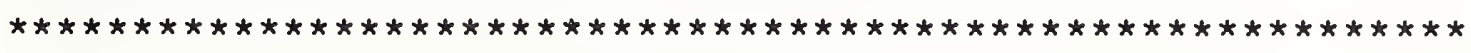


$251 \mathrm{C}$

SUBROUTINES TO SUPPORT THE MAIN PROGRAM.

SUBROUTINE LINE ( $P I$, WAVE, RKO , CRI , ZP, WI , NS, SPAERE,

TRA, XP , DF, LSRIP)

COMPLEX CRI $(0: 21)$

REAL PI, RKO, WAVE, SPAFRE, W( $0: 3$ ), WI (20), PER, PO( 3 ), $Z, Z P(0: 20), N S, T L, X P(20), T R A(2)$

SET THE ARRAYS TO ZERO.

DO $210 \quad I=0,20$

$2 P(I)=0.0$

$\operatorname{CRI}(I)=\operatorname{CMPIX}(0.0,0.0)$

IF(I.GT.0) THEN

$W I(I)=0.0$

$\mathrm{XP}(I)=0.0$

END IF

CONTINUE

READ IN THE WAVELENGTH, THE PERIOD, THE NUMBER OF LAYERS

READ*, WAVE, PER, NS

SPAERE $=1.0 /$ PER

READ IN THE RI'S OF THE AIR LAYER AND THE SUBSTRATE. ID DETERMINES WHETHER THE IINE IS DEFINED BY PARAMETERS OR LAYER BY LAYER.

READ*, CRI ( 0) , CRI ( 2I), ID

READ IN THE WIDTH POLYNOMIAL COEFFICIENTS.

READ*,W(0),W(1),W(2),W(3)

READ IN THE 2 POSITION STARTING POINTS FOR THE 1ST,2ND AND 3RD ORDERS.

READ*,PO( 1 ), $P O(2), P O(3)$

READ IN THE REFRACTIVE INDEX OF THE PATTERNED LAYER AND THE LAYER THICKNESS.

READ*, CRI ( I ), TL

READ IN THE PHOTOMULTIPLIER SLIT WIDTH AND THE VIDEO CAMERA WIDTH PARAMETER. 
301

302

303

304

$305 \mathrm{C}$

$306 \mathrm{C}$

$307 \mathrm{C}$

$308 \mathrm{C}$

$309 \mathrm{C}$

$310 \mathrm{C}$

$311 \mathrm{C}$

$312 \mathrm{C}$

$313 \mathrm{C}$

314

$315 \mathrm{C}$

316

$317 \mathrm{C}$

$318 \mathrm{C}$

$319 \mathrm{C}$

320

321

322

323

324

325

326

327

$328 \mathrm{C}$

$329 \mathrm{C}$

$330 \mathrm{C}$

$331 \mathrm{C}$

$332 \mathrm{C}$

$333 \mathrm{C}$

334

335

$336 \quad 230$

337

$338 \mathrm{C}$

$339 \mathrm{C}$

$340 \mathrm{C}$

341

342

$343 \mathrm{C}$

$344 \mathrm{C}$

345

346

347

348

349

350

READ*, TRA ( 1 ), TRA ( 2 )

IF(TRA(1).GT.0.01) THEN

$\operatorname{TRA}(2)=0.0$

END IF

THE CAMERA WIDTH PARAMETER TRA(2) IS SET TO ZERO WHENEVER THERE IS A FINITE SIIT WIDTH TRA(1).

READ IN THE SKIP PARAMETER (TO SRIP IMAGE CALCULATIONS) AND THE AMOUNT OF DEFOCUS IN NUMBER OF WAVES.

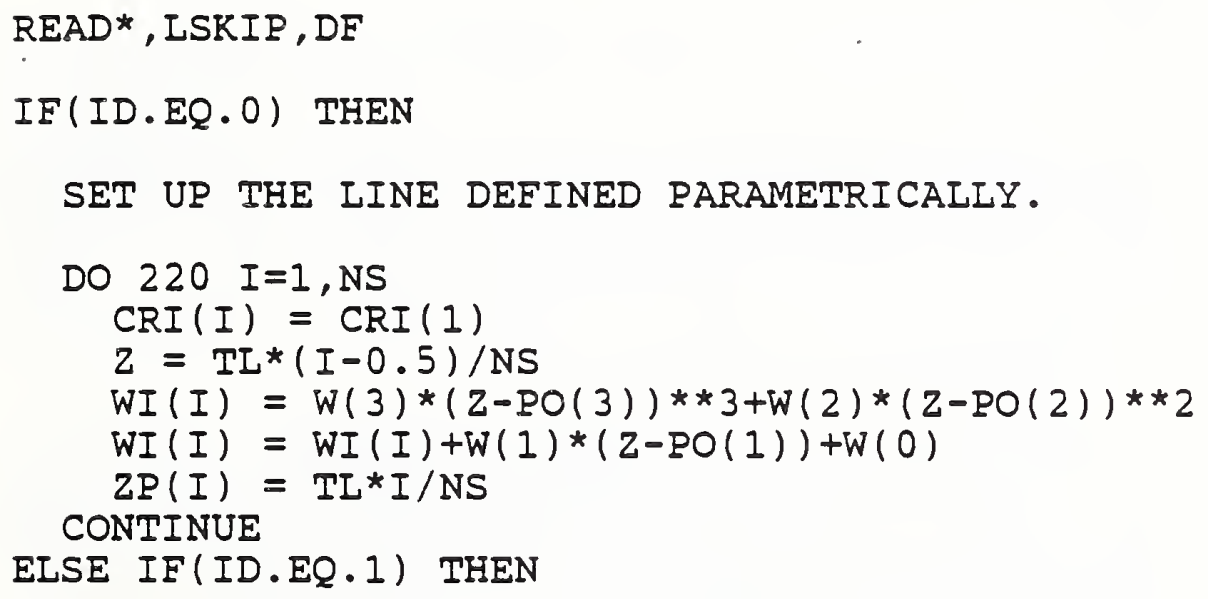

CALCULATE THE WAVE NUMBER.

$\mathrm{RKO}=2.0 * \mathrm{PI} / \mathrm{WAVE}$

SEND PARAMETERS TO A FILE.

$\operatorname{WRITE}(9, *)$ 'RUN PARAMETERS.'

$\operatorname{WRITE}(9, *)$ 'WAVELENGTH = ',WAVE

$\operatorname{WRITE}(9, *)$ ' NUMBER OF LAYERS $=$ ', NS

$\operatorname{WRITE}(9, *)$ 'WAVE NUMBER $=$ ', RKO

$\operatorname{WRITE}(9, *)$ 'AIR LAYER $=$ ', $\operatorname{CRI}(0)$

$\operatorname{WRITE}(9, *)$ 'SUBSTRATE $=$ ', CRI(21) 


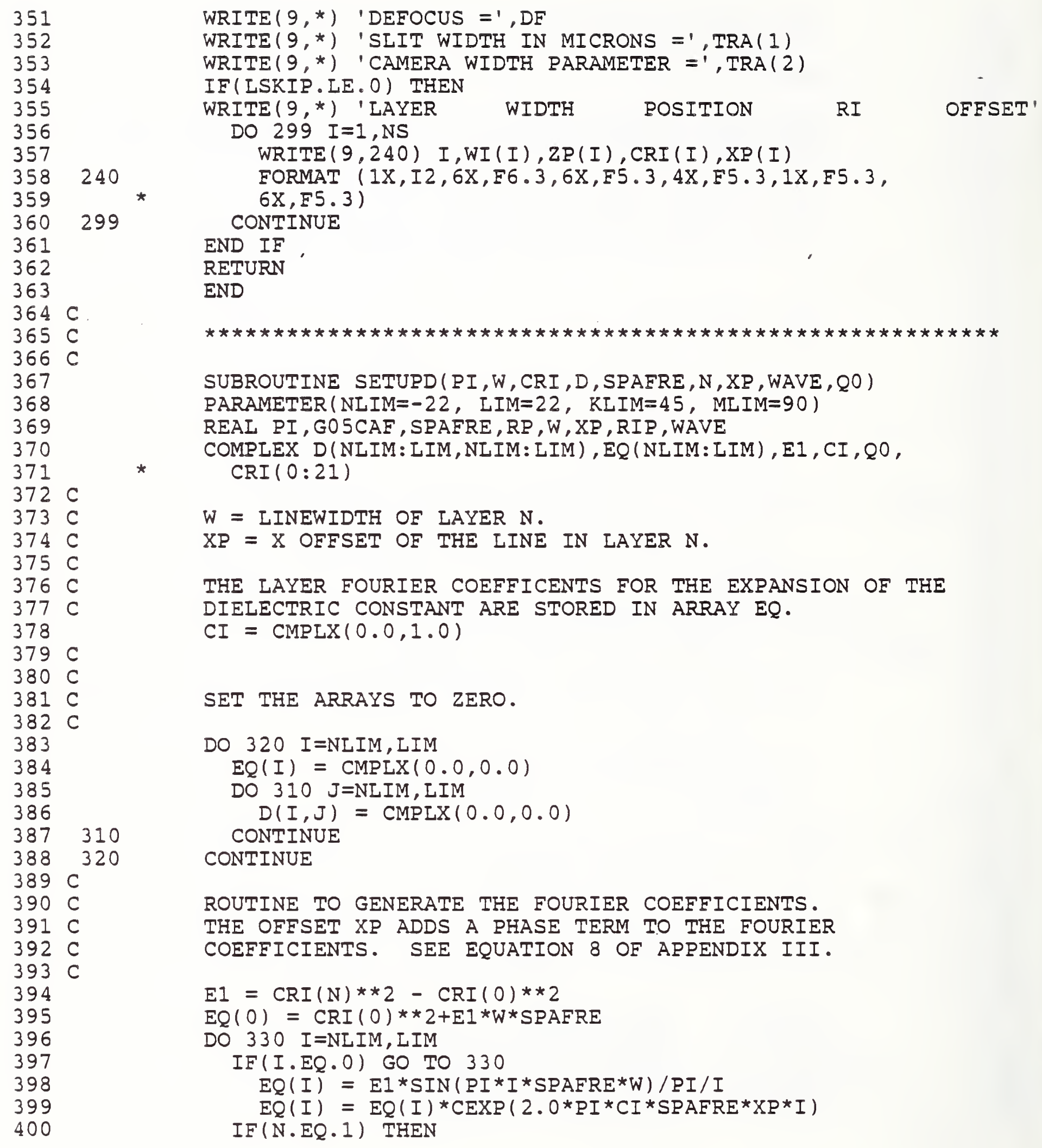

$\operatorname{WRITE}(9, *)$ 'DEFOCUS $=$ ', DF

$\operatorname{WRITE}(9, *)$ 'SLIT WIDTH IN MICRONS $=1, \operatorname{TRA}(1)$

$\operatorname{WRITE}(9, *)$ 'CAMERA WIDTH PARAMETER $=1, \operatorname{TRA}(2)$

IF(LSKIP.LE.0) THEN

$\operatorname{WRITE}(9, *)$ ' LAYER

WIDTH POSITION

RI

OFFSET' DO $299 \mathrm{I}=1$, NS WRITE $(9,240) \quad I, W I(I), Z P(I), C R I(I), X P(I)$

\section{9} FORMAT (1X,I2, 6X, F6.3, 6X,F5.3, 4X, F5.3, 1X, F5.3, $6 \mathrm{X}, \mathrm{F5} .3)$

CONTINUE

END IF

RETURN

END

SUBROUTINE SETUPD ( $P I, W, C R I, D, S P A F R E, N, X P$, WAVE , $Q 0$ )

PARAMETER (NLIM $=-22, \operatorname{LIM}=22, K L I M=45, \operatorname{MLIM}=90$ )

REAL PI, GO5CAF, SPAFRE, RP, W, XP, RIP, WAVE COMPLEX D(NLIM: LIM,NLIM:LIM), EQ(NLIM:LIM),E1,CI,Q0, $\operatorname{CRI}(0: 21)$

$W=$ LINEWIDTH OF LAYER $\mathrm{N}$.

$\mathrm{XP}=\mathrm{X}$ OFFSET OF THE LINE IN LAYER $\mathrm{N}$.

THE LAYER FOURIER COEFFICENTS FOR THE EXPANSION OF THE DIELECTRIC CONSTANT ARE STORED IN ARRAY EQ. $C I=\operatorname{CMPLX}(0.0,1.0)$

SET THE ARRAYS TO ZERO.

ROUTINE TO GENERATE THE FOURIER COEFFICIENTS. THE OFFSET XP ADDS A PHASE TERM TO THE FOURIER COEFFICIENTS. SEE EQUATION 8 OF APPENDIX III. 


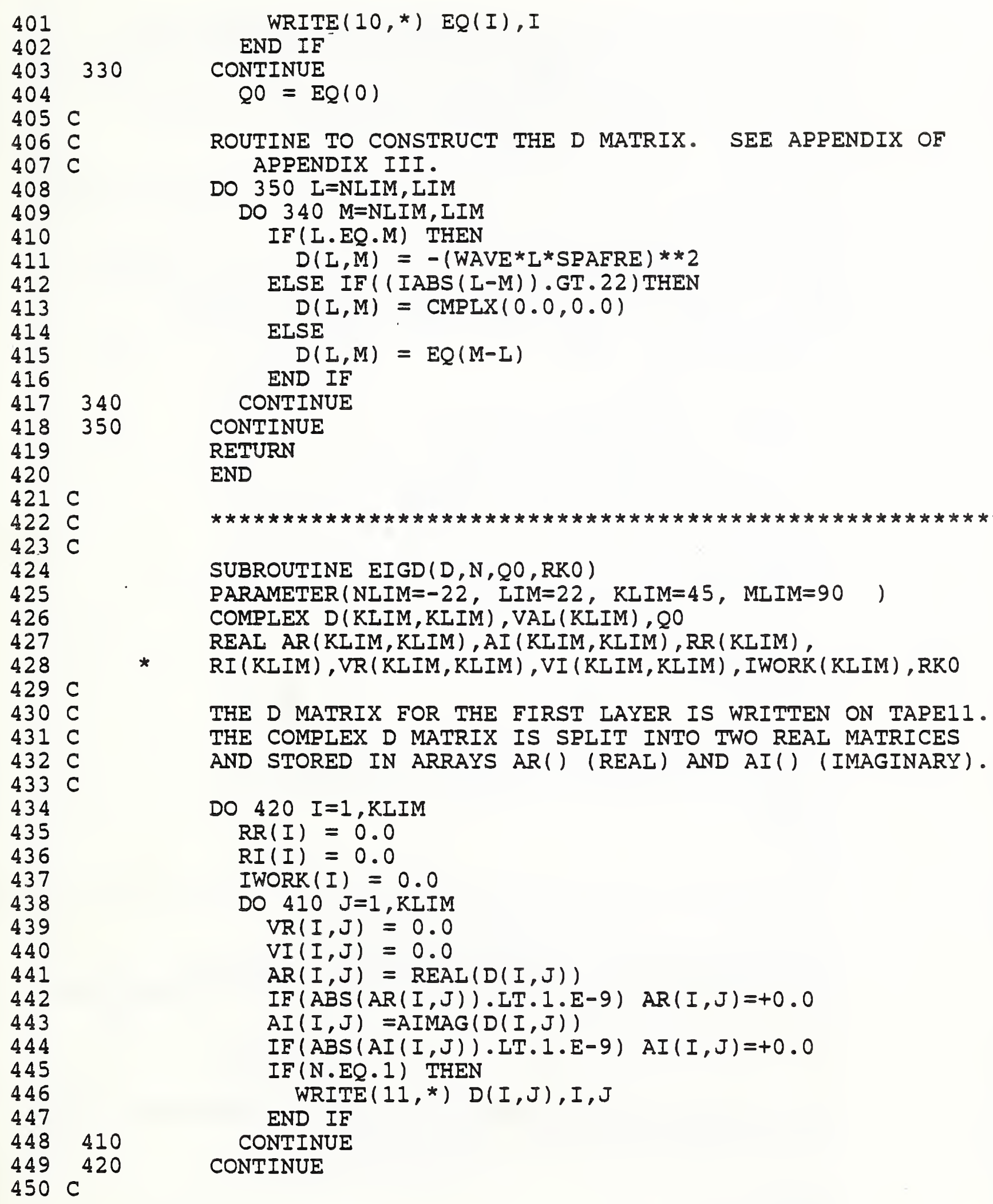




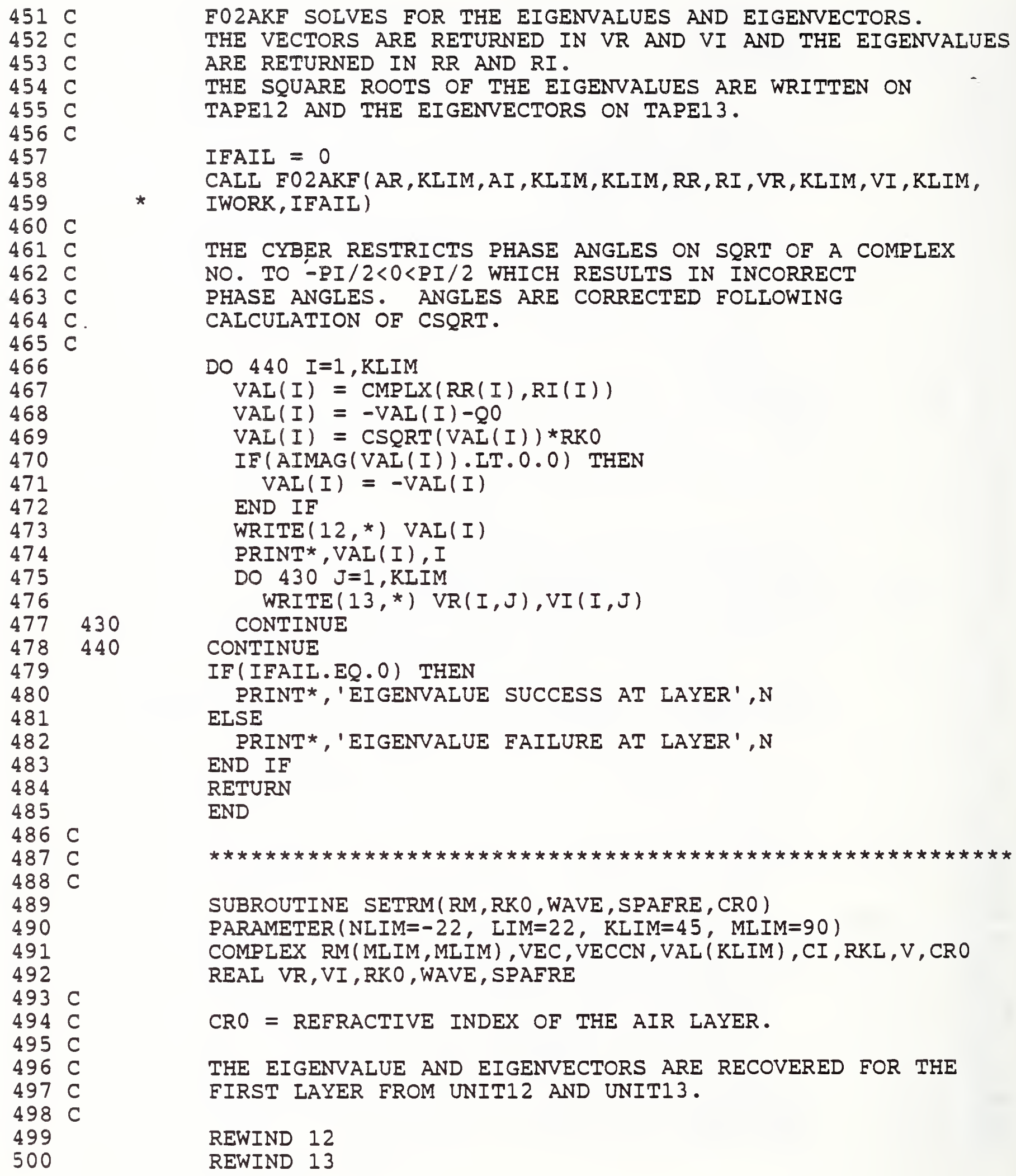

$451 \mathrm{C}$

$452 \mathrm{C}$

$453 \mathrm{C}$

$454 \mathrm{C}$

$455 \mathrm{C}$

$456 \mathrm{C}$

457

458

459

$460 \mathrm{C}$

$461 \mathrm{C}$

$462 \mathrm{C}$

$463 \mathrm{C}$

$464 \mathrm{C}$.

$465 \mathrm{C}$

466

467

468

469

470

471

472

473

474

475

476

477

478

479

480

481

482

483

484

485

$486 \mathrm{C}$

$487 \mathrm{C}$

$488 \mathrm{C}$

489

490

491

492

$493 \mathrm{C}$

$494 \mathrm{C}$

$495 \mathrm{C}$

$496 \mathrm{C}$

$497 \mathrm{C}$

$498 \mathrm{C}$

499

500

F02AKF SOLVES FOR THE EIGENVALUES AND EIGENVECTORS. THE VECTORS ARE RETURNED IN VR AND VI AND THE EIGENVALUES ARE RETURNED IN RR AND RI. THE SQUARE ROOTS OF THE EIGENVALUES ARE WRITTEN ON TAPE12 AND THE EIGENVECTORS ON TAPE13.

IFAIL $=0$

CALI F02ARF (AR, KIIM, AI , KIIM, KLIM, RR, RI , VR, KIIM, VI , KIIM, IWORK, IFAII)

THE CYBER RESTRICTS PHASE ANGLES ON SQRT OF A COMPLEX NO. TO -PI/ $2<0<P I / 2$ WHICH RESUITS IN INCORRECT PHASE ANGLES. ANGLES ARE CORRECTED FOLLOWING CALCULATION OF CSQRT. 
501

502

503

504

505

506

507

508

509

510

511

512

513

514

515

516

517

518

519

520

$521 \mathrm{C}$

522

523

524

525

526

527

$528 \mathrm{C}$

529 C

530

531

532

$533 \mathrm{C}$

$534 \mathrm{C}$

$535 \mathrm{C}$

536

537

538

539

540

541

542

543

544

545

$546 \mathrm{C}$

$547 \mathrm{C}$

$548 \mathrm{C}$

549

550

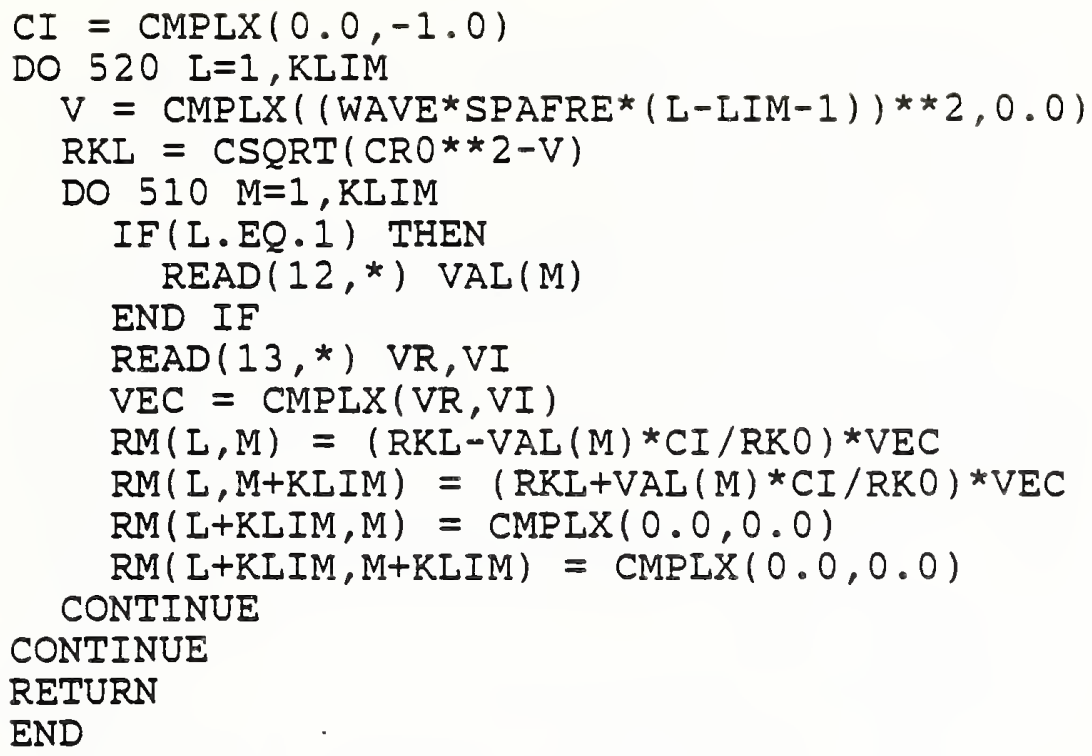

READ PAST THE EIGENVALUES AND EIGENVECTORS RELATING TO THE PREVIOUS LAYERS.

IF (N.GT.1) THEN

DO $630 \quad I=1, N-1$

DO $620 \mathrm{~J}=1, \mathrm{KLIM}$

$\operatorname{READ}(12, *) \operatorname{VAL}(\mathrm{J})$

DO $610 \mathrm{~K}=1, \mathrm{KLIM}$

610

$\operatorname{READ}(13, *) \mathrm{VR}, \mathrm{VI}$

620

630 CONTINUE

CONTINUE

CONTINUE

END IF

SET UP THE UPPER LAYER MATRIX.

DO $650 \mathrm{~L}=1, \mathrm{KLIM}$

DO $640 \mathrm{M}=1$, KLIM 
571680

$572 \quad 685$

$573 \mathrm{C}$

$574 \mathrm{C}$

$575 \mathrm{C}$

$576 \mathrm{C}$

577

578

579

580

581

582

583

584

585

586

587

588

589

$590 \mathrm{C}$

$591 \mathrm{C}$

592

593

594

595

596

597

598

599

600

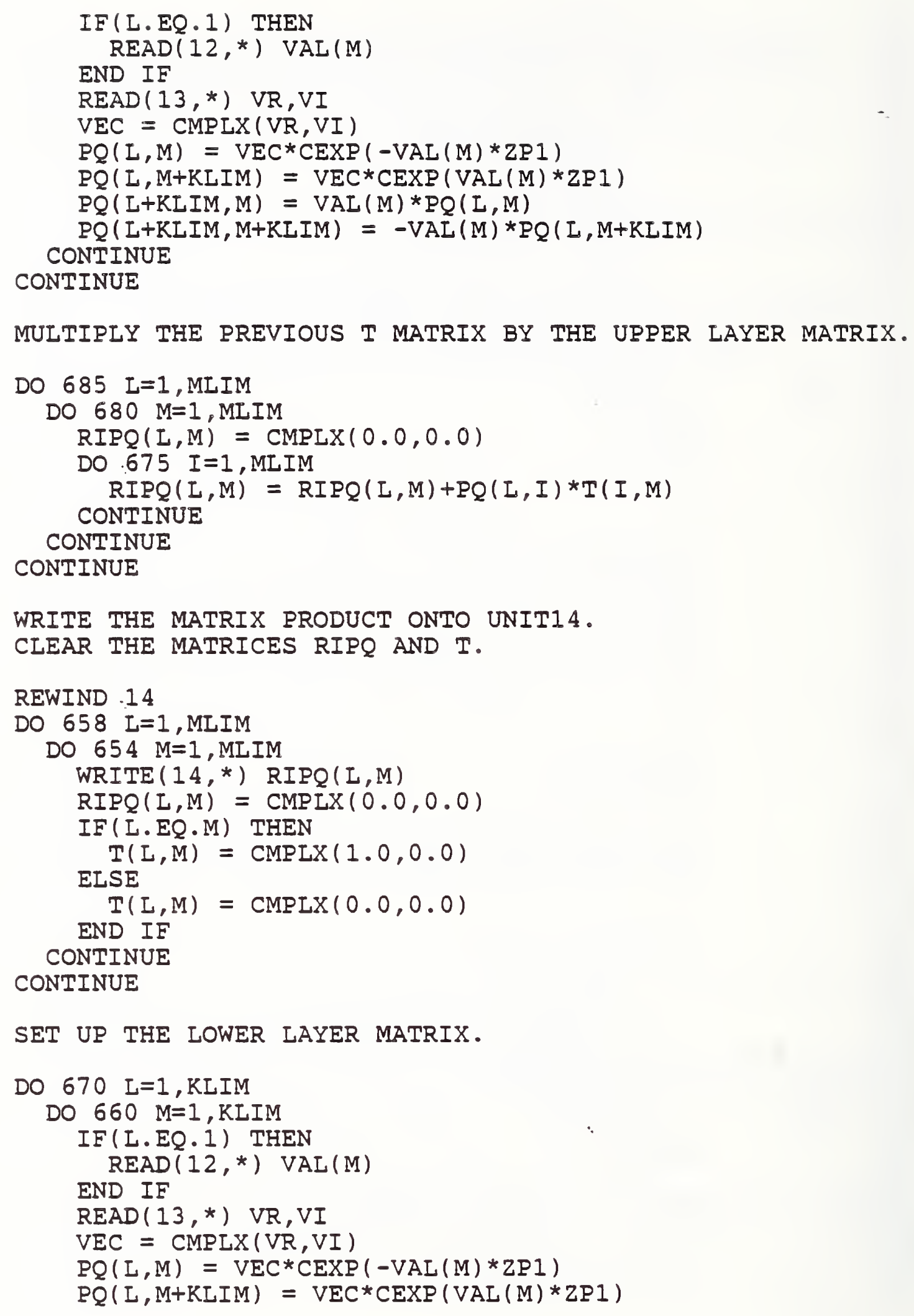


601

602

603660

$604 \quad 670$

$605 \mathrm{C}$

$606 \mathrm{C}$

$607 \mathrm{C}$

$608 \mathrm{C}$

609

610

611

612

613

614

615

616

617

$618 \mathrm{C}$

$619 \mathrm{C}$

$620 \mathrm{C}$

621

622

623

624

$625 \quad 604$

$626 \quad 608$

$627 \mathrm{C}$

$628 \mathrm{C}$

$629 \mathrm{C}$

630

631

632

633

634

635690

$636 \quad 694$

$637 \quad 698$

638

639

$640 \mathrm{C}$

$641 \mathrm{C}$

$642 \mathrm{C}$

643

644

645

646

$647 \mathrm{C}$

$648 \mathrm{C}$

$649 \mathrm{C}$

$650 \mathrm{C}$

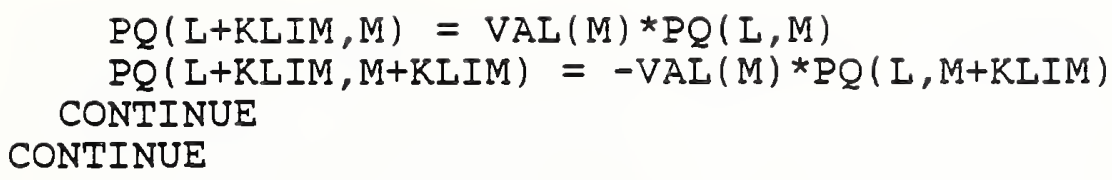

FO 4ADF INVERTS THE MATRIX PQ AND RETURNS THE INVERSE IN THE MATRIX RIPQ.

IFAIL $=0$

CALL FO 4ADF ( $P Q$, MLIM, T, MLIM, MLIM, MLIM, RIPQ, MLIM, WKSPCE, IFAIL)

IF(IFAIL.EQ.O) THEN

PRINT*, 'SUCCESSFUL MATRIX INVERSION AT INTERFACE', N ELSE

PRINT* ' SINGULAR MATRIX AT INTERFACE', N

STOP

END IF

RECOVER THE PREVIOUS MATRIX FROM UNITI4.

REWIND 14

DO 608 L=1, MLIM

DO $604 \mathrm{M}=1$, MLIM

$\operatorname{READ}(14, *) \quad \mathrm{PQ}(L, M)$

CONTINUE

CONTINUE

MULTIPLY THE TWO MATRICES AND RETURN THE PRODUCT IN $T$.

DO $694 \quad L=1, M L I M$

DO $694 \mathrm{M}=1$, MLIM

$T(L, M)=\operatorname{CMPLX}(0.0,0.0)$

DO $690 \quad I=1, M L I M$

$T(I, M)=T(L, M)+R I P Q(I, I) * P Q(I, M)$

CONTINUE

CONTINUE

CONTINUE

RETURN

END

SUBROUTINE SETRN(RN, NS, ZS,VAL)

PARAMETER (NLIM=-22, LIM=22, KLIM=45, MLIM=90)

COMPLEX RN (KLIM, MLIM) , VEC, VECCN , VAL(KLIM)

REAI VR, VI, ZS, NS

$Z S=$ THE $Z$ POSITION OF THE SUBSTRATE INTERFACE.

CRS = THE COMPLEX REFRACTIVE INDEX OF THE SUBSTRATE. 
701

702

703

704

705

706

707

708

709

710

711

712

713

714

715

716

717

718

719

720

721

722

723

724

725

726

727

728

729

730

731

732

733

734

$735 \mathrm{C}$

$736 \mathrm{C}$

$737 \mathrm{C}$

738

739

740

$741 \quad 840$

742

$743 \mathrm{C}$

$744 \mathrm{C}$

$745 \mathrm{C}$

$746 \mathrm{C}$

$747 \mathrm{C}$

748

749 C

750

810

820
IF(NS.EQ.1) THEN

DO $820 \mathrm{~L}=1$, KLIM

$V=\operatorname{CMPLX}(($ WAVE $* \operatorname{SPAFRE} *(L-L I M-1)) * * 2,0.0)$

$\mathrm{S} 2=\operatorname{CSQRT}(\mathrm{CRSUB} * * 2-\mathrm{V})$

S3 $=\operatorname{CSQRT}(C R S * \star 2-V)$

$A 1=\operatorname{CEXP}(\operatorname{CMPLX}(0.0,2.0) * R K 0 * T S U B * S 2)$

ASUB $=((S 2+S 3)-(S 2-S 3) * A 1) /\left((S 2+S 3)+(S 2-S 3) * A_{1}\right)$

$\mathrm{RKI}=\mathrm{ASUB}{ }^{*} \mathrm{~S} 2$

DO $810 \mathrm{M}=1$, KIIM

$$
R M(I+K I I M, M)=R N(I, M) *(R K L+V A L(M) * C I / R K O)
$$

$R M(I+K L I M, M+K L I M)=R N(L, M+K L I M) *(R R L-V A L(M) * C I / R K O)$ CONTINUE

ELSE

DO $836 L=1$, KLIM

$V=\operatorname{CMPLX}(($ WAVE*SPAFRE* $(L-L I M-1)) * * 2,0.0)$

$\mathrm{S} 2=\operatorname{CSQRT}($ CRSUB $* * 2-V)$

S3 $=\operatorname{CSQRT}(C R S * * 2-V)$

$A 1=\operatorname{CEXP}\left(\operatorname{CMPLX}(0.0,2.0) * \operatorname{RKO} * \operatorname{TSUB}^{*}{ }^{\mathrm{S}} \mathrm{S} 2\right)$

$A S U B=((S 2+S 3)-(S 2-S 3) * A 1) /((S 2+S 3)+(S 2-S 3) * A 1)$

$\mathrm{RKL}=\mathrm{ASUB} * \mathrm{~S} 2$

DO $834 \mathrm{M}=1$, KIIM

DO $832 I=1, R L I M$

$R M(L+K L I M, M)=R M(L+K I I M, M)+R K L *(R N(I, I) * T(I, M)+$

$R N(I, I+K I I M) * T(I+K L I M, M))+(R N(I, I) * V A I(I) * C I / R K O *$ $T(I, M)-R N(I, I+K I I M) * V A I(I) * C I / R K O * T(I+K L I M, M))$

$R M(L+K L I M, M+K L I M)=R M(L+R I I M, M+R I I M)+R K L *(R N(L, I)$ * $T(I, M+K L I M)+R N(I, I+K L I M) * T(I+K L I M, M+K L I M))+$ $(R N(I, I) * V A L(I) * C I / R K O * T(I, M+K L I M)-R N(I, I+K L I M)$ * $\operatorname{VAL}(I) * C I / R K O * T(I+K I I M, M+R I I M))$

832

834

836 CONTINUE

CONTINUE

CONTINUE

END IF

SET UP THE RIGHT HAND SIDE VECTOR RV.

DO $840 \quad L=1$, MLIM

WKSPCE $(L)=0.0$

$\operatorname{RV}(L)=\operatorname{CMPLX}(0.0,0.0)$

CONTINUE

$\operatorname{RV}(\operatorname{LIM}+1)=\operatorname{CMPLX}(2,0,0.0)$

FO4ADE SOLVES THE SET OF SIMULTANEOUS EQUATIONS DEFINED BY RM AND RV. SEE EQ. 21 OF APPENDIX III.

THE SOLUTIONS ARE RETURNED IN THE VECTOR AV.

IFAIL $=0$

PRINT *, ' 4 SECTIONS OE RM MATRIX' 
$751 \mathrm{C}$

752

$753 \mathrm{C}$

754

$755 \mathrm{C}$

756

757

$758 \mathrm{C}$

759

760

$761 \mathrm{C}$

762

763

$764 \mathrm{C}$

$765 \mathrm{C}$

766

767

768

769

770

771

772

773

$774 \mathrm{C}$

775

776

777

$778 \mathrm{C}$

$779 \mathrm{C}$

780

781

782

783

784

$785 \mathrm{C}$

$786 \mathrm{C}$

$787 \mathrm{C}$

$788 \mathrm{C}$

$789 \mathrm{C}$

790

791

792

793

794

795910

$796 \quad 920$

797 C

$798 \mathrm{C}$

$799 \mathrm{C}$

$800 \mathrm{C}$

WRITE $(1, *)$ 'FIRST SECTION RM(1,M), M=1,45'

PRINT * , $(\operatorname{RM}(1, M), M=1,45)$

$\operatorname{WRITE}(1, *)(\operatorname{RM}(1, M), M=1,45)$

PRINT *, $(\operatorname{RM}(1, M+45), M=1,45)$

$\operatorname{WRITE}(1, *)$ ' $\operatorname{SECOND} \operatorname{SECTION} \operatorname{RM}(1, \mathrm{M}+45), \mathrm{M}=1,45$ '

$\operatorname{WRITE}(1, *)(\operatorname{RM}(1, M+45), M=1,45)$

$\operatorname{PRINT} *,(\operatorname{RM}(46, M), M=1,45)$

WRITE $(1, *)$ 'THIRD SECTION RM( $46, M), M=1,45$ '

$\operatorname{WRITE}(1, *)(\operatorname{RM}(46, M), M=1,45)$

PRINT *, ( RM $(46, M+45), M=1,45)$

$\operatorname{WRITE}(1, *)$ 'FOURTH SECTION RM $(46, \mathrm{M}+45), \mathrm{M}=1,45$ '

$\operatorname{WRITE}(1, *)(\operatorname{RM}(46, M+45), M=1,45)$

IFAIL $=0$

CALI FO 4ADF (RM, MLIM, RV , MLIM, MLIM, 1, AV , MLIM, WKSPCE, IFAIL)

IF(IFAIL.EQ.0) THEN

PRINT*,'SUCCESSFUL SOLUTION FOR (A) COEFFICIENTS.' ELSE

PRINT*, 'FAILURE TO SOLVE FOR (A) COEFFICIENTS.' END IF

PRINT *, ( $\left.A V(L), I,{ }^{\prime} A V^{\prime}, L=1, M L I M\right)$

RETURN

END

SUBROUTINE FCOEFF(AV,FC, T)

PARAMETER ( $N L I M=-22, \quad L I M=22, \quad K L I M=45, \operatorname{MLIM}=90)$

COMPLEX AV (MLIM), FC (KLIM), T(MLIM, MLIM)

REAL VR, VI

REWIND 13

CALCULATE THE FOURIER COEFFICIENTS OF THE PSEUDO-OBJECT AND WRITE THE RESULTS ON UNIT15.

STORE THE FIRST LAYER EIGENVECTORS IN MATRIX T.

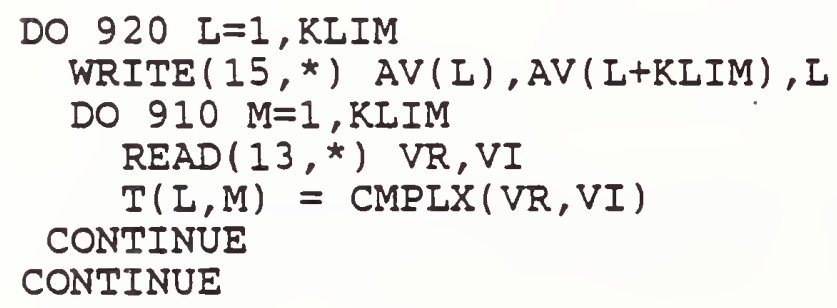

CLEAR THE FC VECTOR AND SET THE FC(0) VALUE TO -1.0 . CALCULATE FOURIER SERIES COEFFICIENTS FC USING THE ACOEFFICIENTS AND THE EIGENVECTORS. SEE EQ. 22 OF APPENDIX III. 
$801 \mathrm{C}$

DO $940 \mathrm{~L}=1, \mathrm{KLIM}$

803

IF(L.EQ.LIM+1) THEN

804

805

806

807

ELSE $F C(L)=\operatorname{CMPLX}(-1.0,0.0)$

808

809

810930

811940

$812 \mathrm{C}$

$813 \mathrm{C}$

$814 \mathrm{C}$

$815 \mathrm{C}$

$816 \mathrm{C}$

817

818

819

820950 $F C(L)=\operatorname{CMPLX}(0.0,0.0)$

END IF

DO $930 \mathrm{M}=1$, KLIM

$$
F C(L)=F C(L)+(A V(M)+A V(M+K L I M)) * T(L, M)
$$

CONTINUE

CONTINUE

THE FOURIER SERIES FOR THE PSEUDO-OBJECT IS WRITTEN ON UNIT16, IN A FORMAT WHICH ALLOWS IT TO INTERFACE TO A PLANAR IMAGING OR OTHER PROGRAM.

DO $950 \mathrm{~K}=1$, KLIM

WRITE $(16,999) \quad F C(K)$

PRINT*,$F C(K)$

CONTINUE

FORMAT (1X,2F20.8)

821.999

RETURN

END

823

$824 \mathrm{C}$

$825 \mathrm{C}$

$826 \mathrm{C}$

SUBROUTINE IMAGE (FC, PI, SPAFRE, TRA, DF, LSKIP, WAVE)

827

828

PARAMETER (NLIM $=-22, \quad L I M=22, \quad K L I M=45, \quad M L I M=90$ )

REAL PI, WAVE, SPAFRE, RR, RI, XPOS, TRA (2),

829

830

$\operatorname{RIM}(-600: 600), \operatorname{IR}, \operatorname{CAM}(-100: 100), \operatorname{ELEC}(-500: 500), X, Y N, X I$

831

$832 \mathrm{C}$

$833 \mathrm{C}$

$834 \mathrm{C}$

$835 \mathrm{C}$

$836 \mathrm{C}$

$837 \mathrm{C}$

$838 \mathrm{C}$

839

$840 \mathrm{C}$

$841 \mathrm{C}$

$842 C$

843

844

845

846

$847 \quad 105$

2

COMPLEX FC(NLIM:LIM), CI, CCI, RT, FFC,FOC (-KLIM:KLIM)

THIS SUBROUTINE COMPUTES THE IMAGE OF THE LINE ASSUMING ONE DIMENSIONAL OPTICS.

THE IMAGE IS COMPUTED FOR 1000 POINTS OVER A. RANGE $O F+1-0.5 * T H E$ PERIOD.

$C I=\operatorname{CMPLX}(0.0,1.0)$

IF LSKIP $=1$ THEN READ IN THE PREVIOUS COEFFICIENTS.

IF(LSKIP.EQ.1) THEN

DO 105 I=NLIM, LIM

$\operatorname{READ}(16, *) \operatorname{RR}, \operatorname{RI}$

$F C(I)=\operatorname{CMPLX}(R R, R I)$

848

$849 C$

$850 \mathrm{C}$

CONTINUE

END IF

OBJ = NUMERICAL A.PERTURE OF THE OBJECTIVE. 


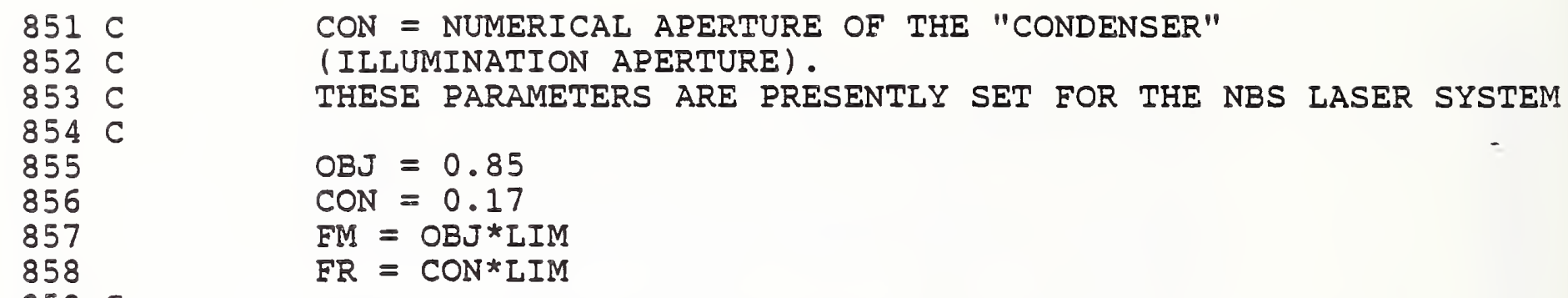

THE DEFOCUS TERM DE ADDS A PHASE MODULATION TO THE FOURIER SERIES.

863

$C C I=C I * 2.0 * P I * S P A F R E$

864

865

866

$867 \mathrm{C}$

DO $115 I=-$ RLIM, KIIM

$\operatorname{FOC}(I)=\operatorname{CEXP}(2.0 * P I * C I * D F *($ WAVE*I*SPAFRE $) * * 2)$ CONTINUE

THE IMAGE IS COMPUTED FOR AN ADDITIONAL 100 POINTS ON EITHER SIDE IN ORDER TO ALLOW FOR CONVOLUTION WITH A DETECTOR APERTURE OR IMPULSE RESPONSE.

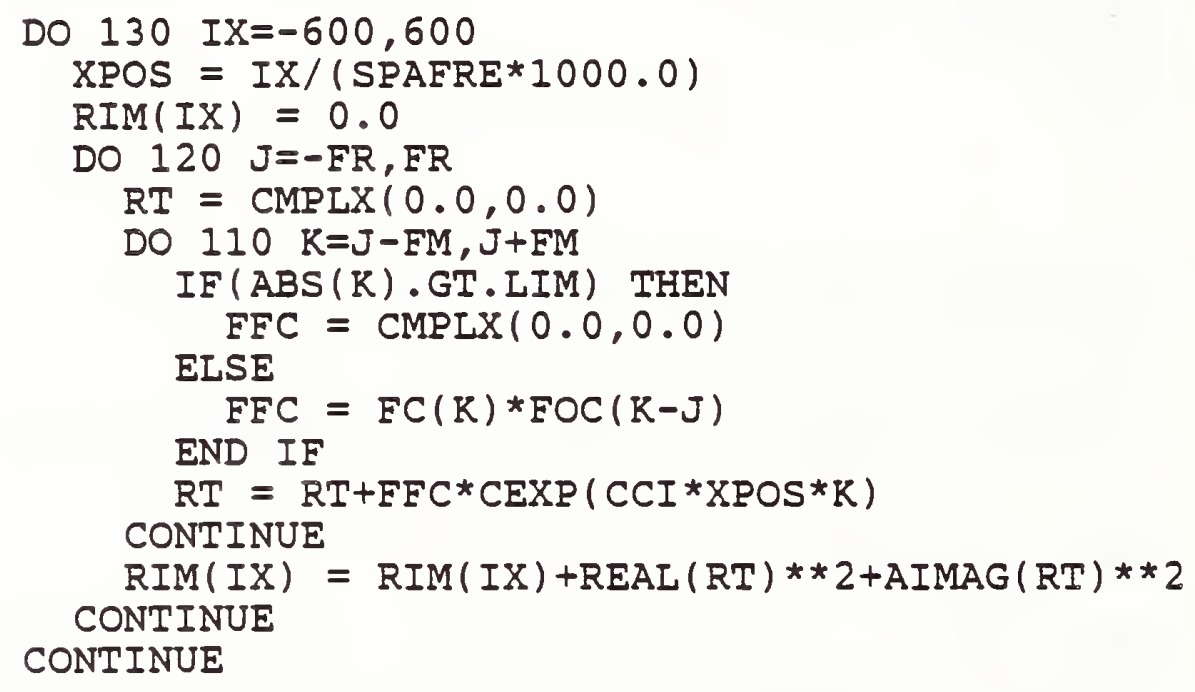

THE ARRAY RIM() CONTAINS THE OPTICAL IMAGE PROFILE.

CONVOLVE THE IMAGE WITH THE DETECTOR LINE SPREAD FUNCTION.

IF TRA(1) IS GREATER THAN 0.01 THEN A SLIT APERTURE IS ASSUMED.

OTHERWISE IF TRA(2) IS GREATER THAN 0.05 THEN A VIDEO CAMERA WITH A GAUSSIAN LINE SPREAD FUNCTION IS ASSUMED. IF BOTH ARE ZERO THEN NO CONVOLUTION TARES PLACE. THE ARRAY CAM() CONTAINS THE VIDEO CAMERA RESPONSE. THE ARRAY ELEC() CONTAINS THE ELECTRICAL OUTPUT SIGNAL OF THE TRANSDUER. 


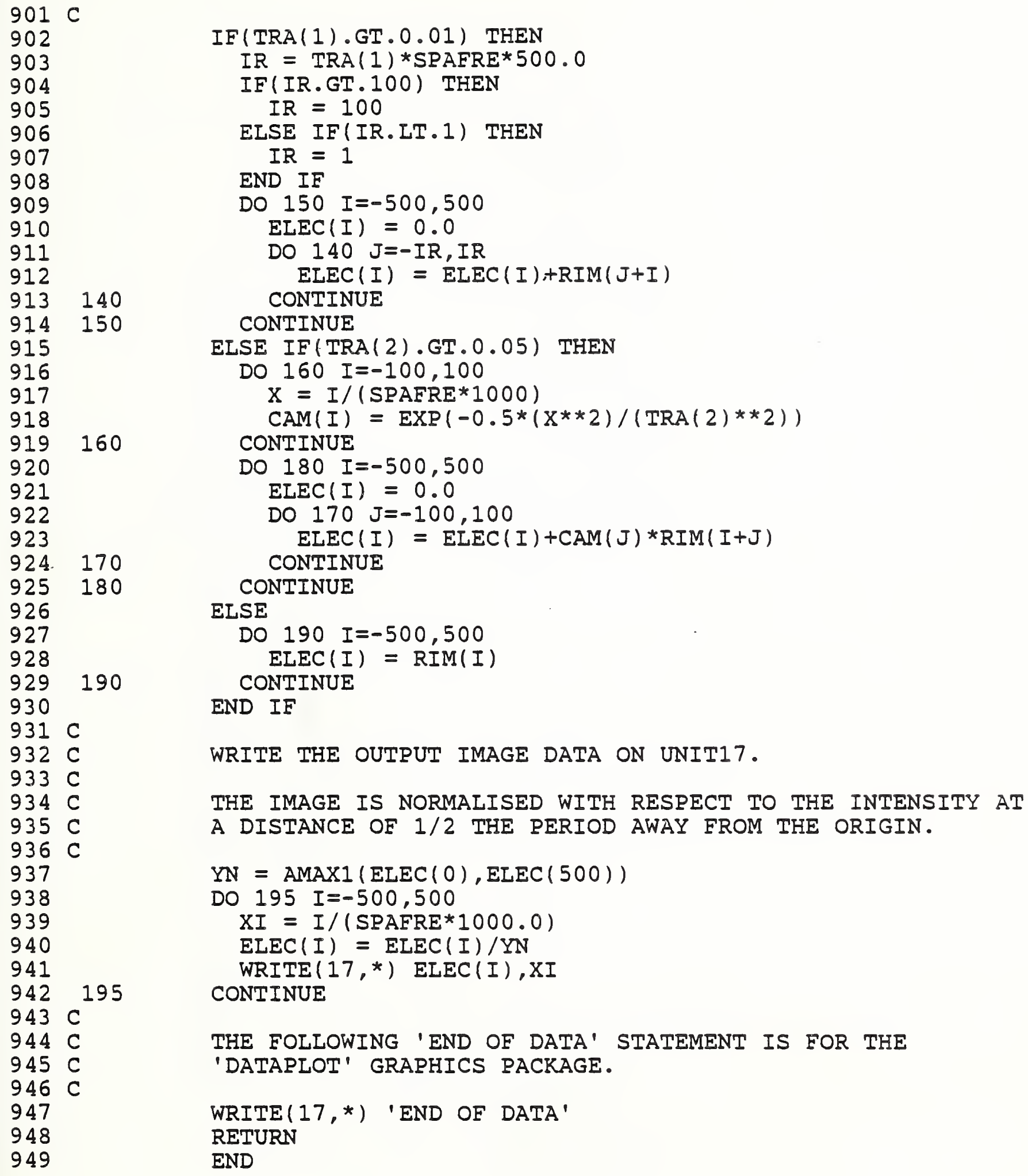

WRITE THE OUTPUT IMAGE DATA ON UNIT17.

THE IMAGE IS NORMALISED WITH RESPECT TO THE INTENSITY AT A DISTANCE OF $1 / 2$ THE PERIOD AWAY FROM THE ORIGIN.

$Y N=\operatorname{AMAX} 1(\operatorname{ELEC}(0), \operatorname{ELEC}(500))$

DO $195 I=-500,500$

$X I=I /($ SPAFRE*1000.0)

$\operatorname{ELEC}(I)=\operatorname{ELEC}(I) / Y N$

WRITE $(17, *)$ ELEC (I),XI

CONTINUE

THE FOLLOWING 'END OF DATA' STATEMENT IS FOR THE 'DATAPLOT' GRAPHICS PACKAGE.

WRITE $(17, *)$ 'END OF DATA' RETURN

END 
Appendix II

Test Cases for Thick Layer Imaging Program (THRIMAG) 

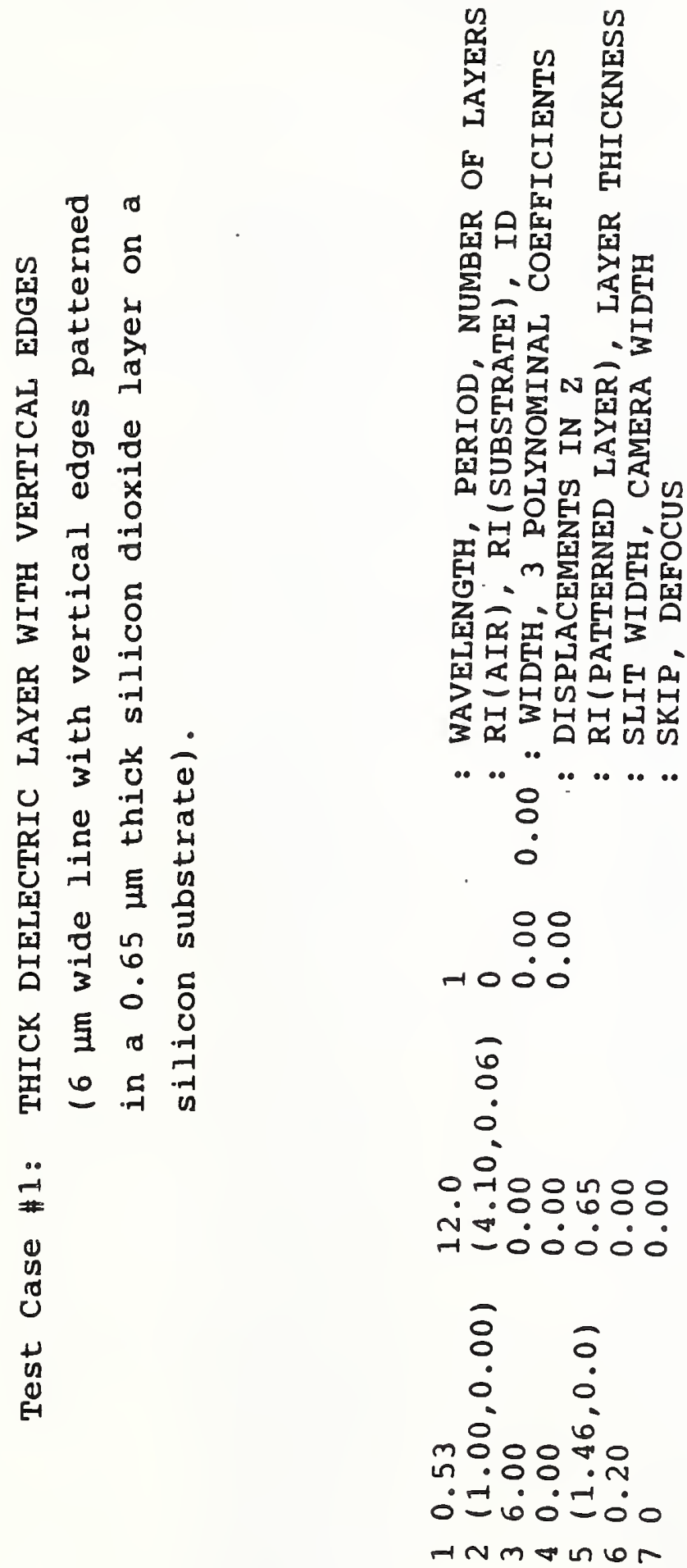
Test Case \#1: File FCOFPO: Fourier Coefficients of the Pseudo-Object

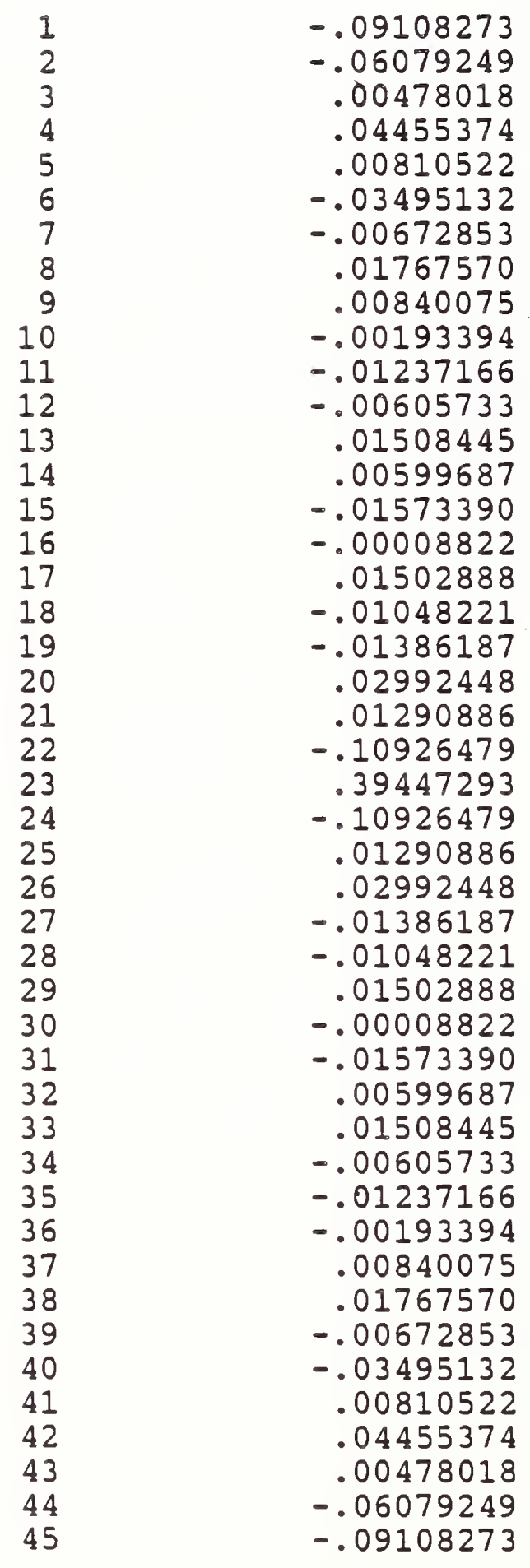

$$
\begin{array}{r}
.00519622 \\
.01308535 \\
.01658667 \\
.00100212 \\
-.00558479 \\
-.01667204 \\
.00298502 \\
.02462243 \\
-.00504291 \\
-.02052349 \\
.00493059 \\
.00957640 \\
-.00253726 \\
.00264588 \\
-.00030316 \\
-.01386078 \\
.00264068 \\
.02557344 \\
-.00424937 \\
-.04556850 \\
.00491631 \\
.13891567 \\
.04366360 \\
.13891567 \\
.00491631 \\
-.04556850 \\
-.00424937 \\
.02557344 \\
.00264068 \\
-.01386078 \\
-.00030316 \\
.00264588 \\
-.00253726 \\
.00957640 \\
.00493059 \\
-.02052349 \\
-.00504291 \\
.02462243 \\
.00298502 \\
-.01667204 \\
-.00558479 \\
.00100212 \\
.01658667 \\
.01308535 \\
.00519622
\end{array}
$$




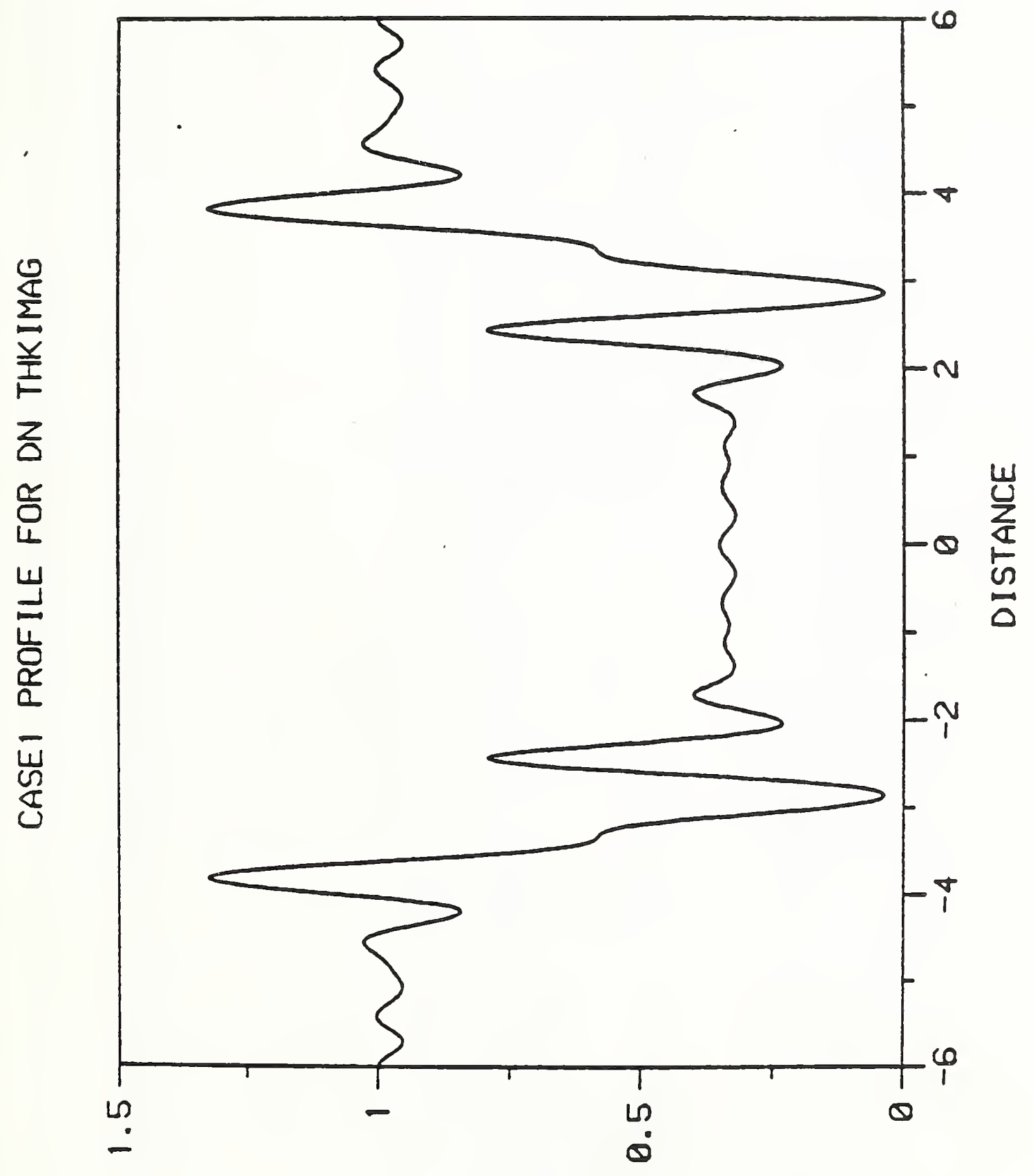




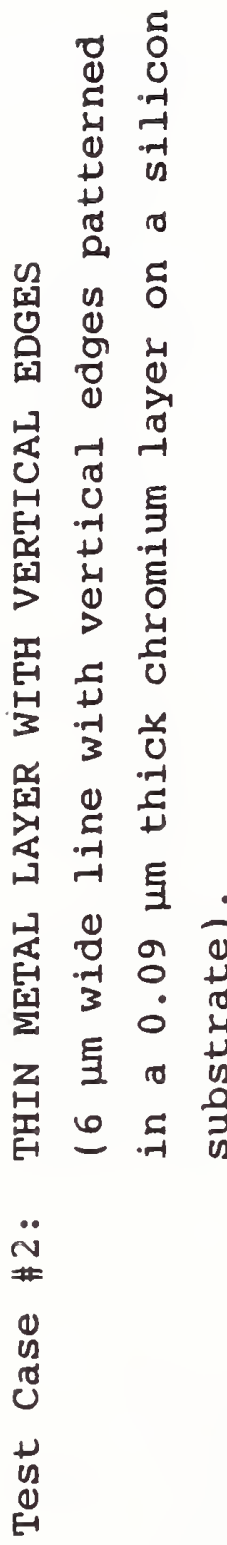

况

品田卧

品.0 弐要

呈四梂

动的

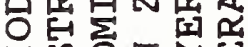

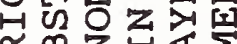

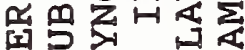

ณ U

田品星星。

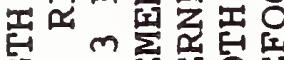

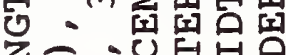

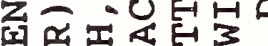

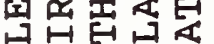

国造员的

$>-H \backsim H$

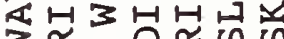

उ响口的
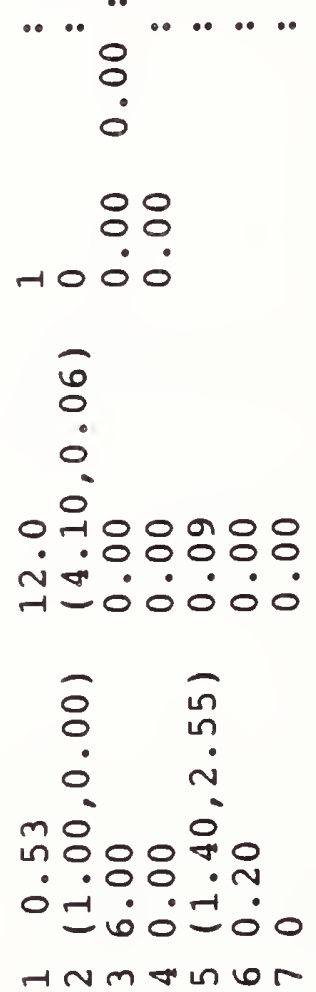
Test Case \#2: File FCOFPO: Fourier Coefficients of the Pseudo-Object

$\begin{array}{lrr}1 & .02598611 & -.01092292 \\ 2 & -.01353529 & .00904450 \\ 3 & -.01731358 & .00311739 \\ 4 & .01756685 & -.00795909 \\ 5 & .01356888 & -.00057359 \\ 6 & -.02047819 & .00686085 \\ 7 & -.01107900 & -.00064297 \\ 8 & .02327938 & -.00598552 \\ 9 & .00921602 & .00126586 \\ 10 & -.02648625 & .00536360 \\ 11 & -.00773381 & -.00157148 \\ 12 & .03061512 & -.00501271 \\ 13 & .00650267 & .00169434 \\ 14 & -.03646409 & .00498365 \\ 15 & -.00544231 & -.00171108 \\ 16 & .04564310 & -.00541449 \\ 17 & .00449766 & .00167165 \\ 18 & -.06227954 & .00669869 \\ 19 & -.00362787 & -.00161367 \\ 20 & .00149616 & -.01032568 \\ 21 & .00279663 & .00156888 \\ 22 & -.29965151 & .02984478 \\ 23 & -.14235833 & -.06645156 \\ 24 & -.29965151 & .02984478 \\ 25 & .00279663 & .00156888 \\ 26 & .00149616 & -.01032568 \\ 27 & -.00362787 & -.00161367 \\ 28 & -.06227954 & .00669869 \\ 29 & .00449766 & .00167165 \\ 30 & .04564310 & -.00541449 \\ 31 & -.00544231 & -.00171108 \\ 32 & -.03646409 & .00498365 \\ 33 & .00650267 & .00169434 \\ 34 & .03061512 & -.00501271 \\ 35 & -.00773381 & -.00157148 \\ 36 & -.02648625 & .00536360 \\ 37 & .00921602 & .00126586 \\ 38 & .02327938 & -.00598552 \\ 39 & -.01107900 & -.00064297 \\ 40 & -.02047819 & .00686085 \\ 41 & .01356888 & -.00057359 \\ 42 & .01756685 & -.00795909 \\ 43 & -.01731358 & .00311739 \\ 44 & -.01353529 & \\ 45 & .02598611 & \end{array}$




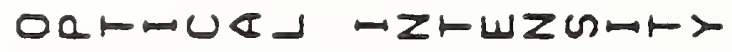

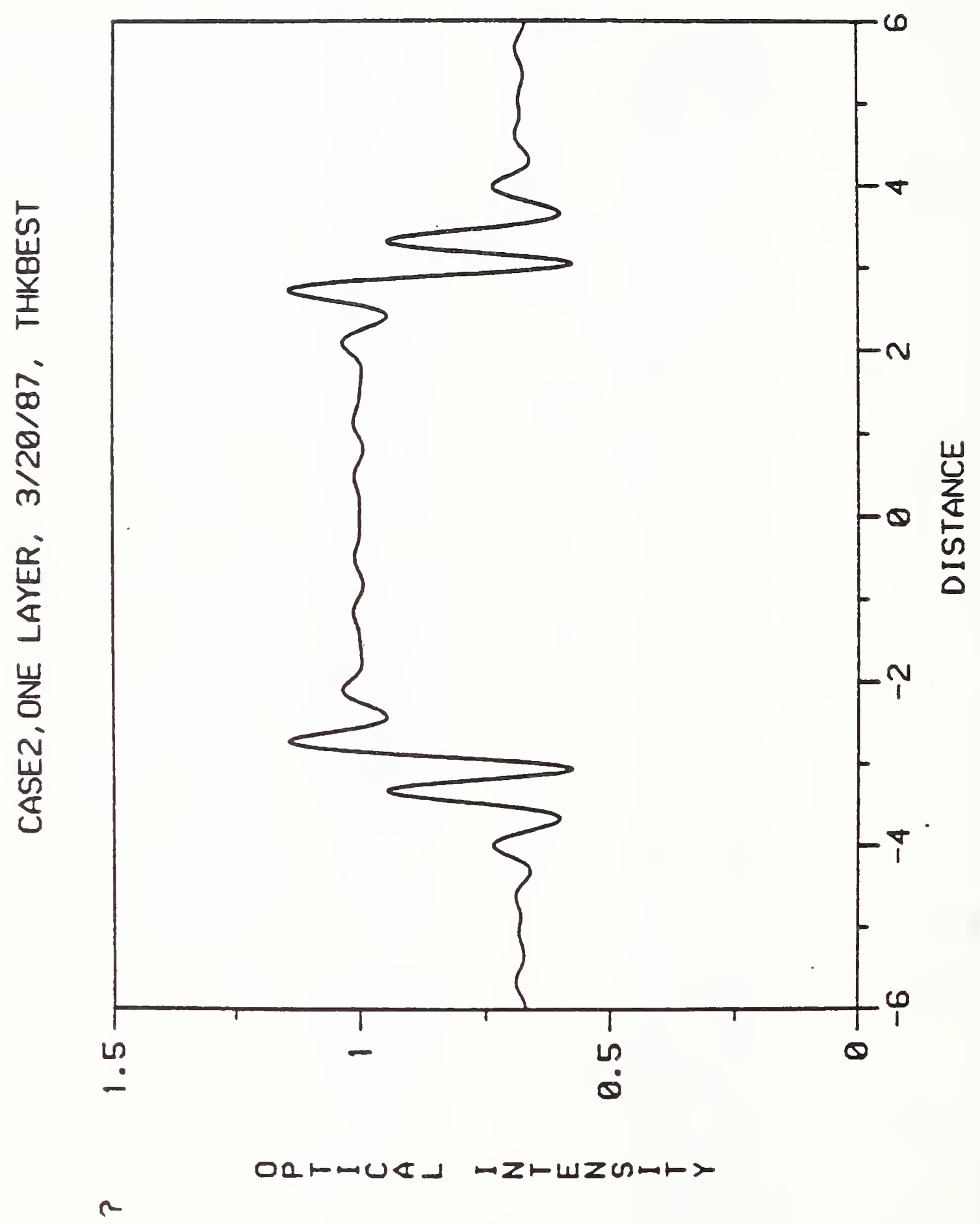




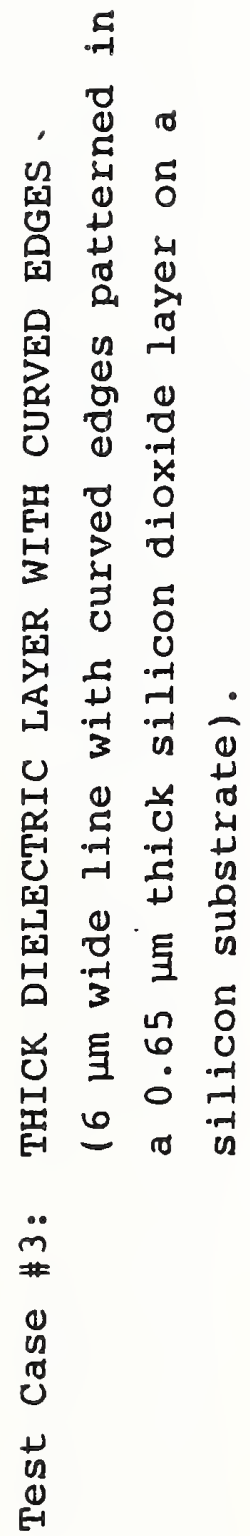

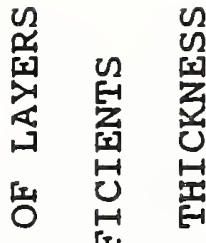

$\widetilde{\sim} \cap$ II $\alpha$

[둄 [x

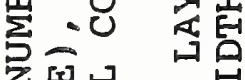

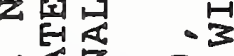

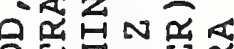

○织回

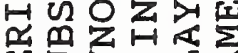

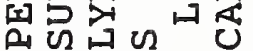

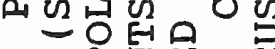

-日只回包

焉响焉焉

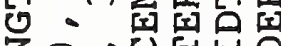

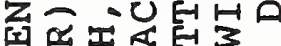

踏我

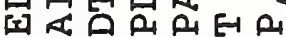

$3-H U=H$

ॐ范

.• ••

0

80

a 0 No
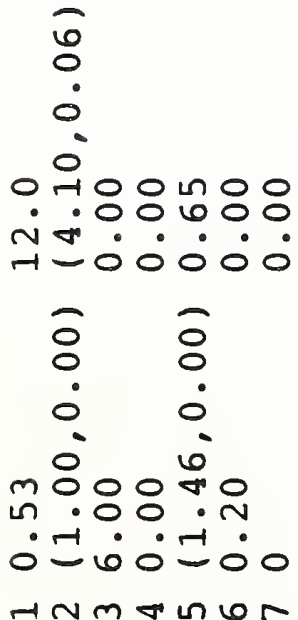
Test Case \#3: File PARFII: Parameter File created

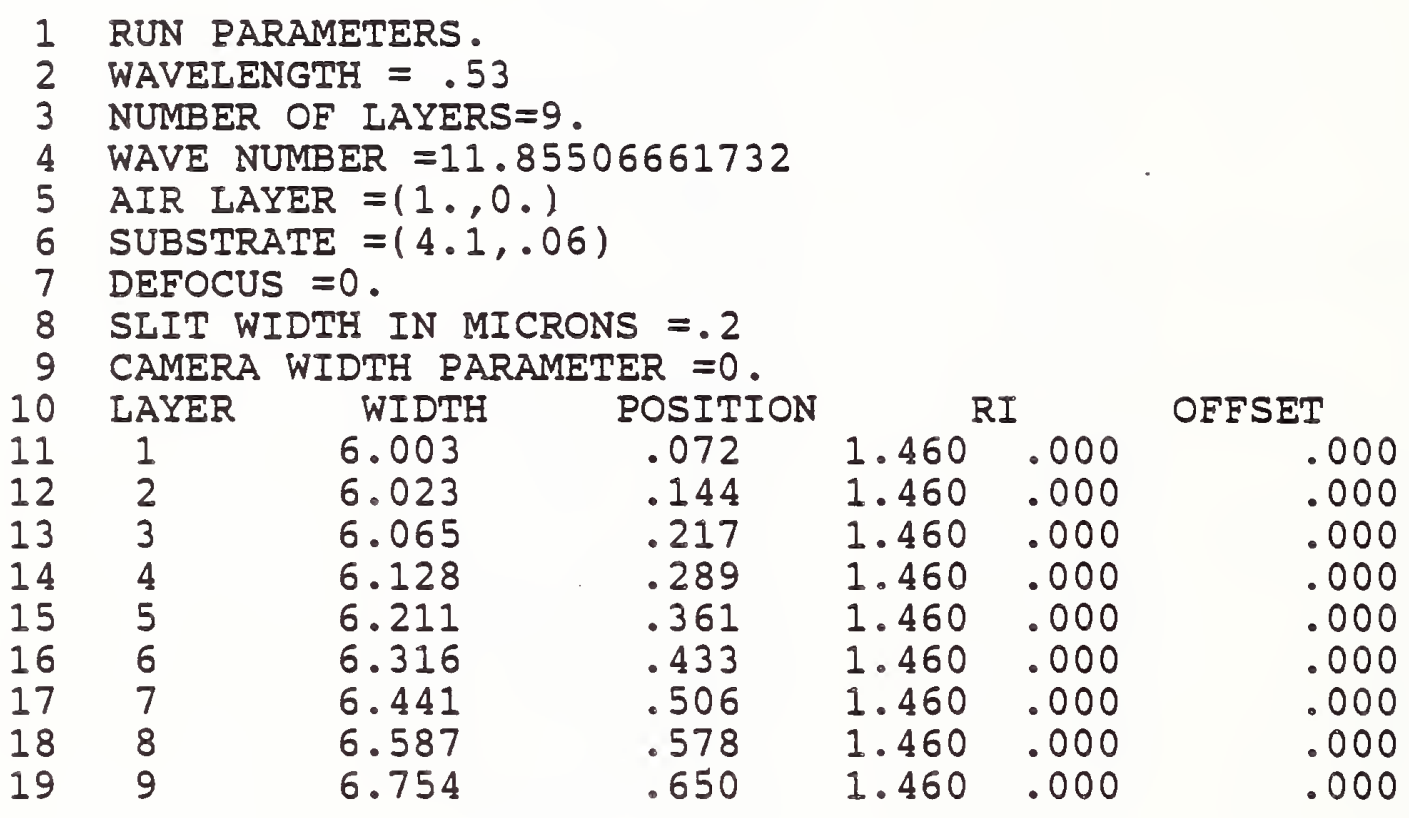


Test Case \#3: File FCOFPO: Fourier Coefficients of the Pseudo-object

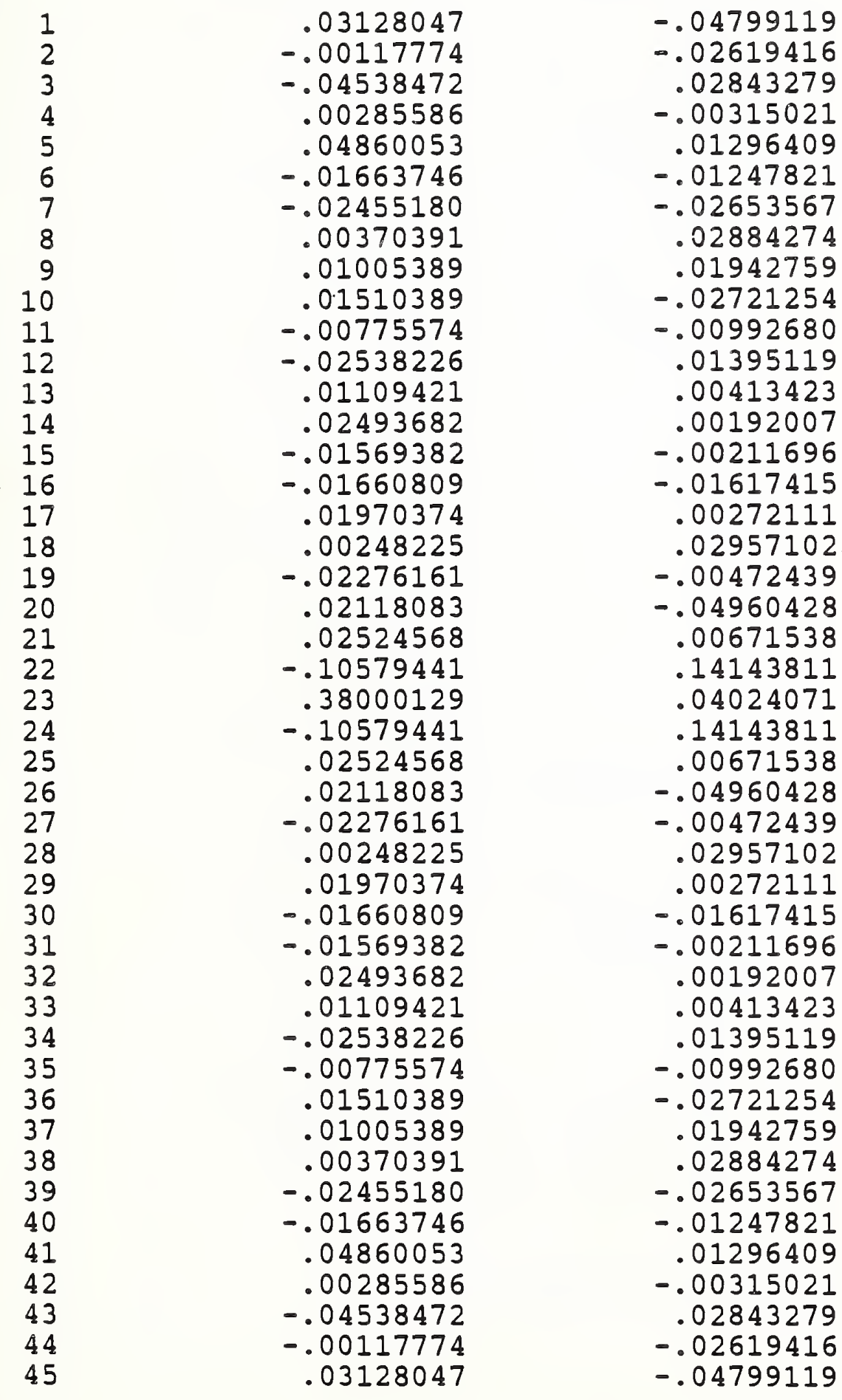




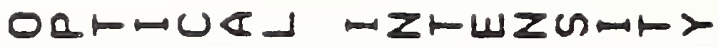

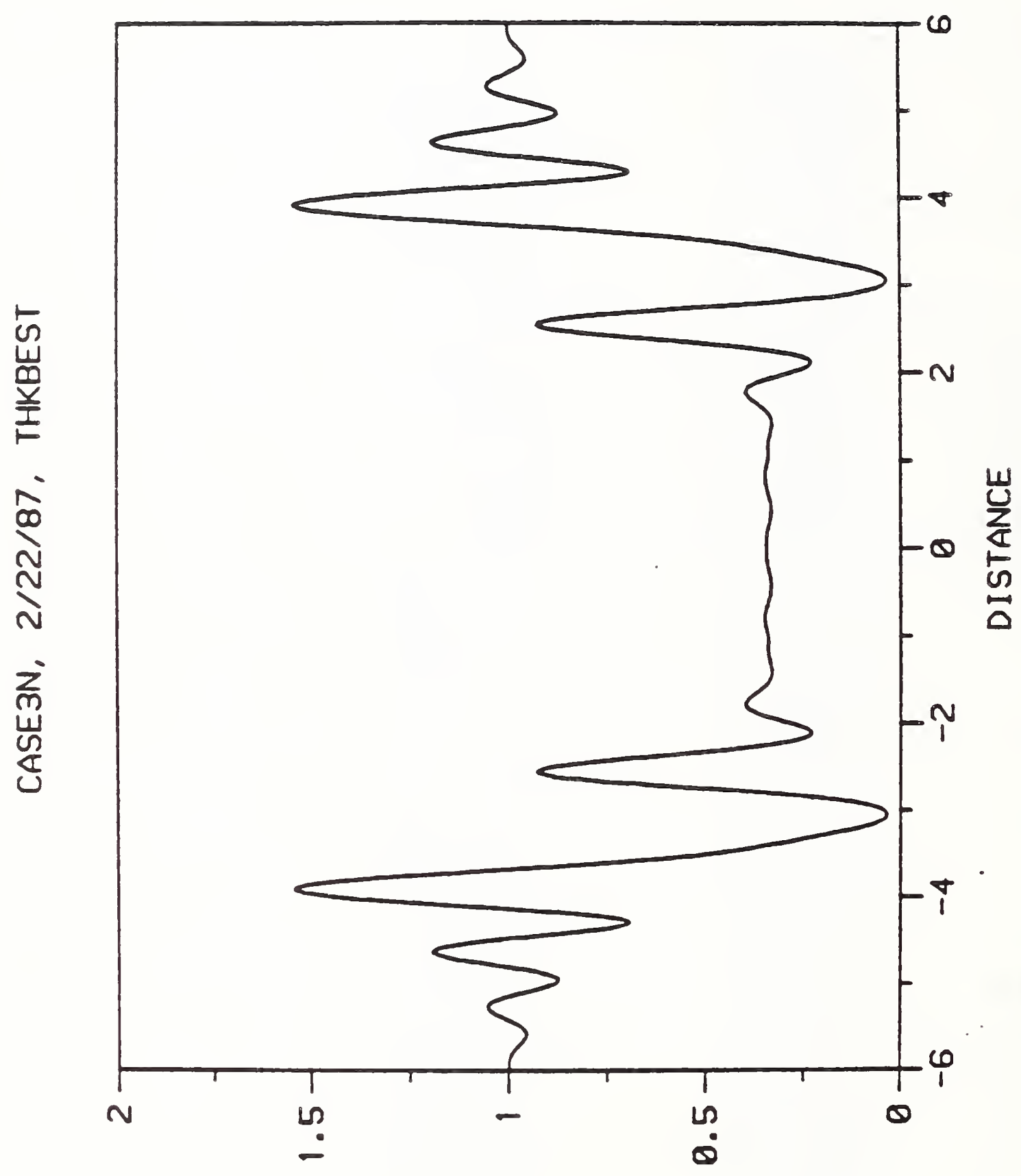

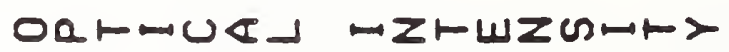

r 


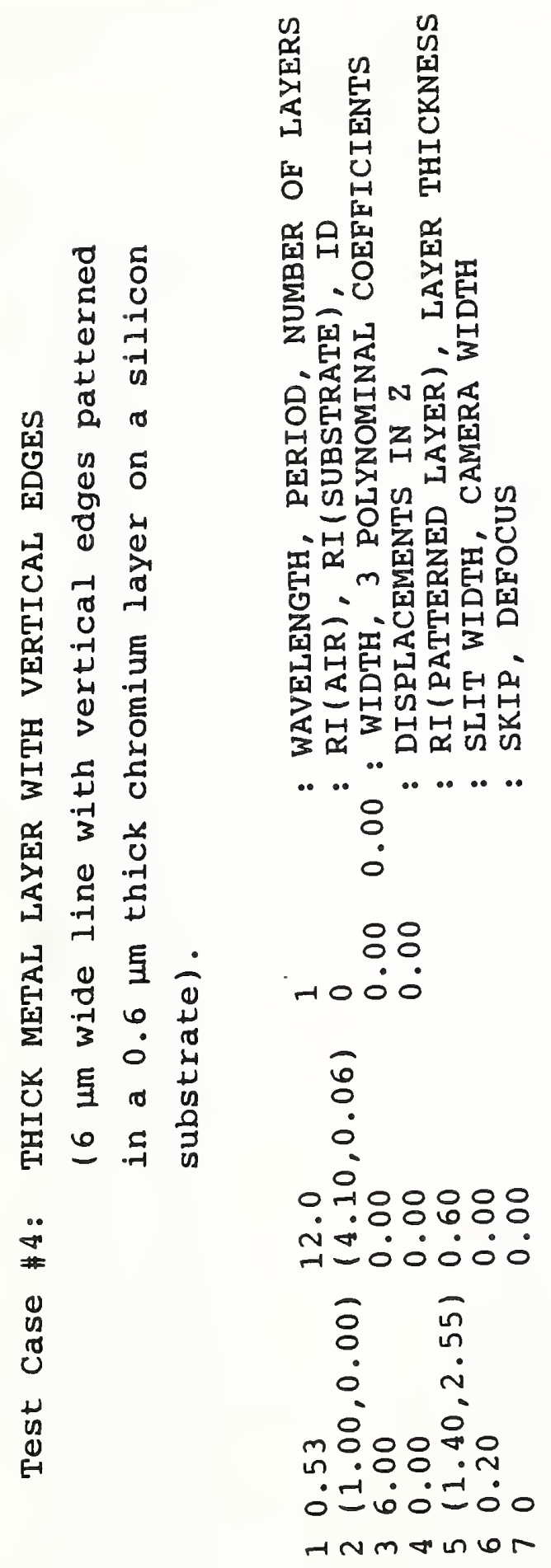


Test Case \#4: File FCOFPO: Fourier Coefficients of the Pseudo-object

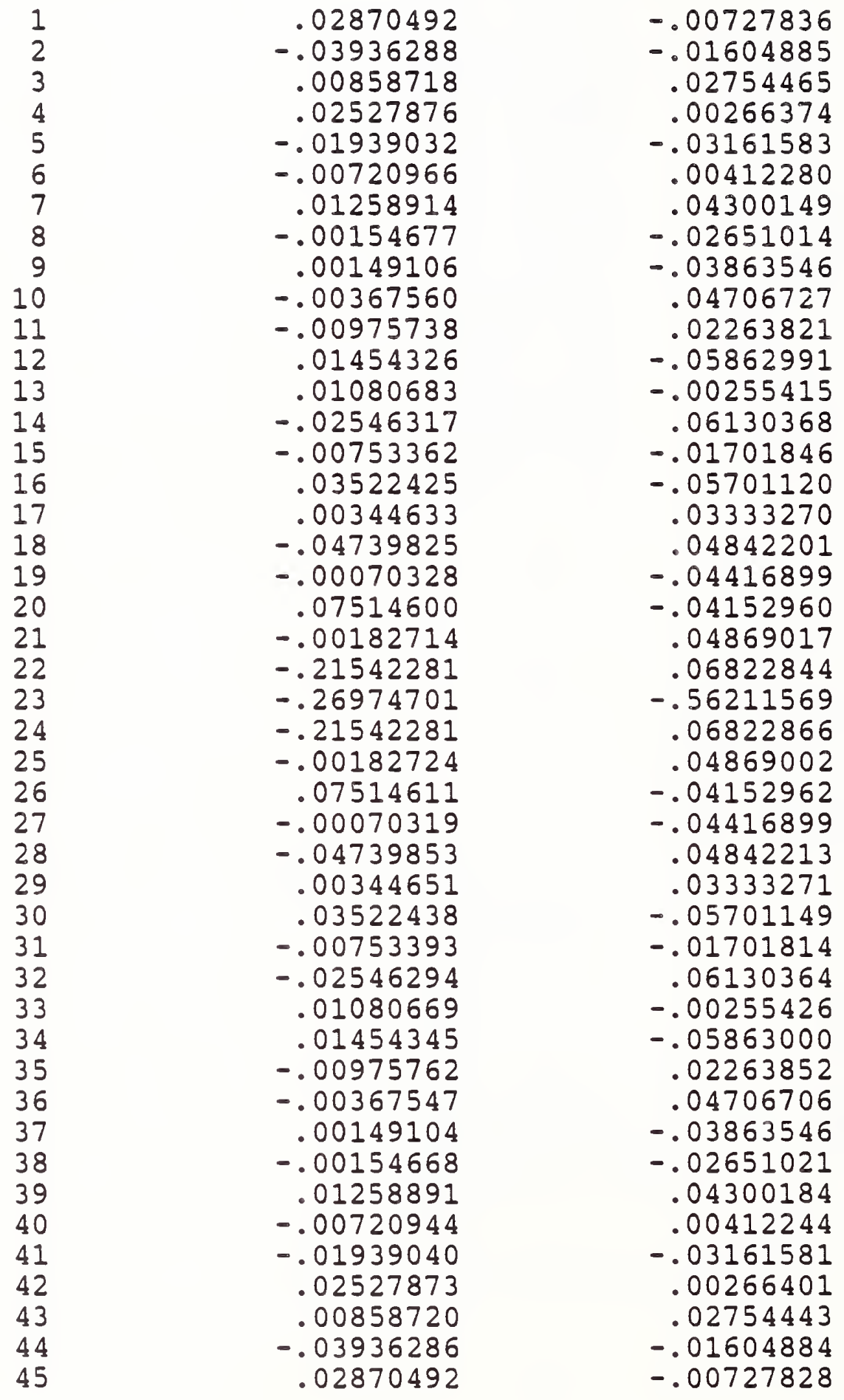


Oat-U氏」

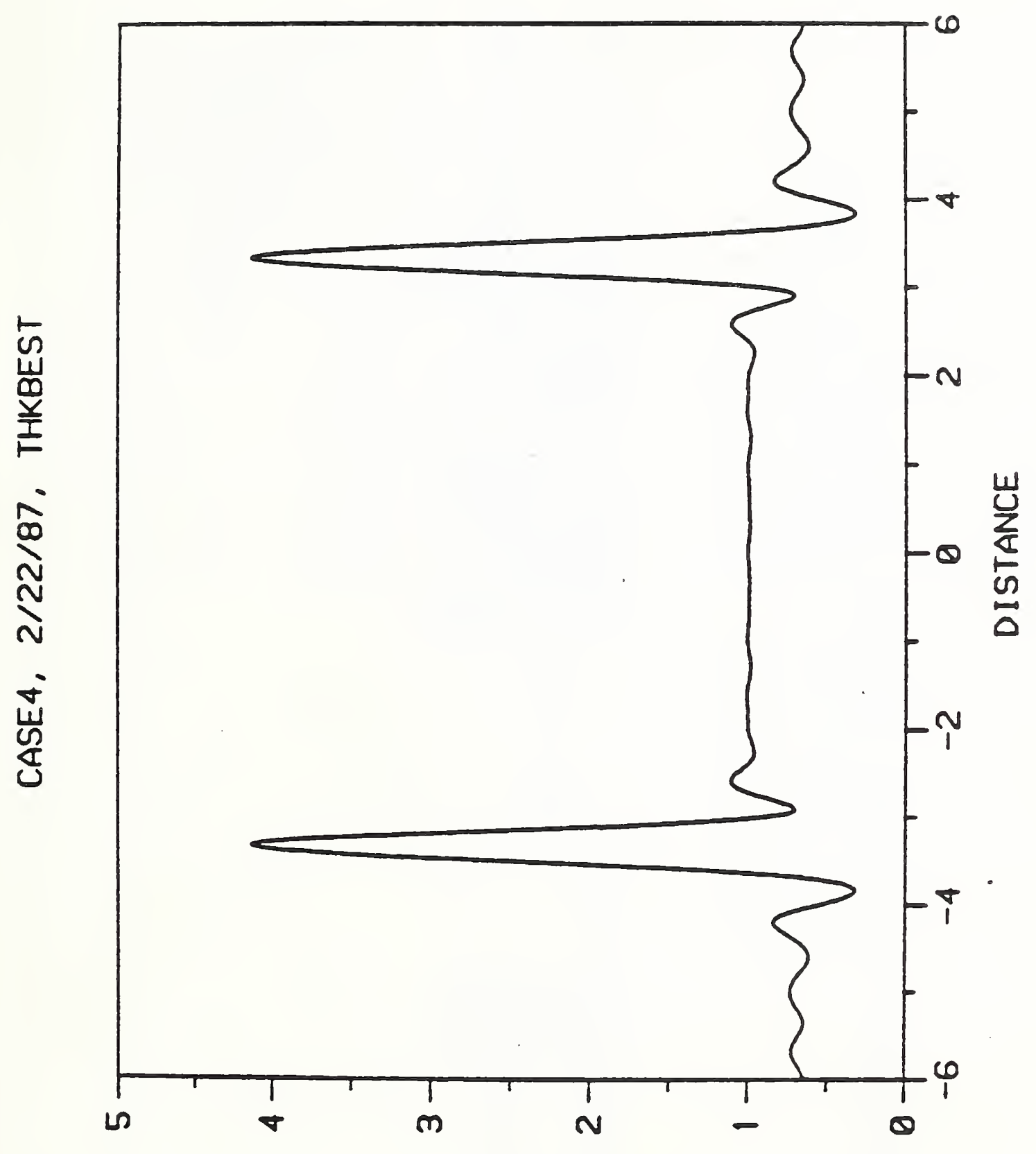

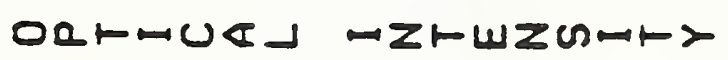

r 


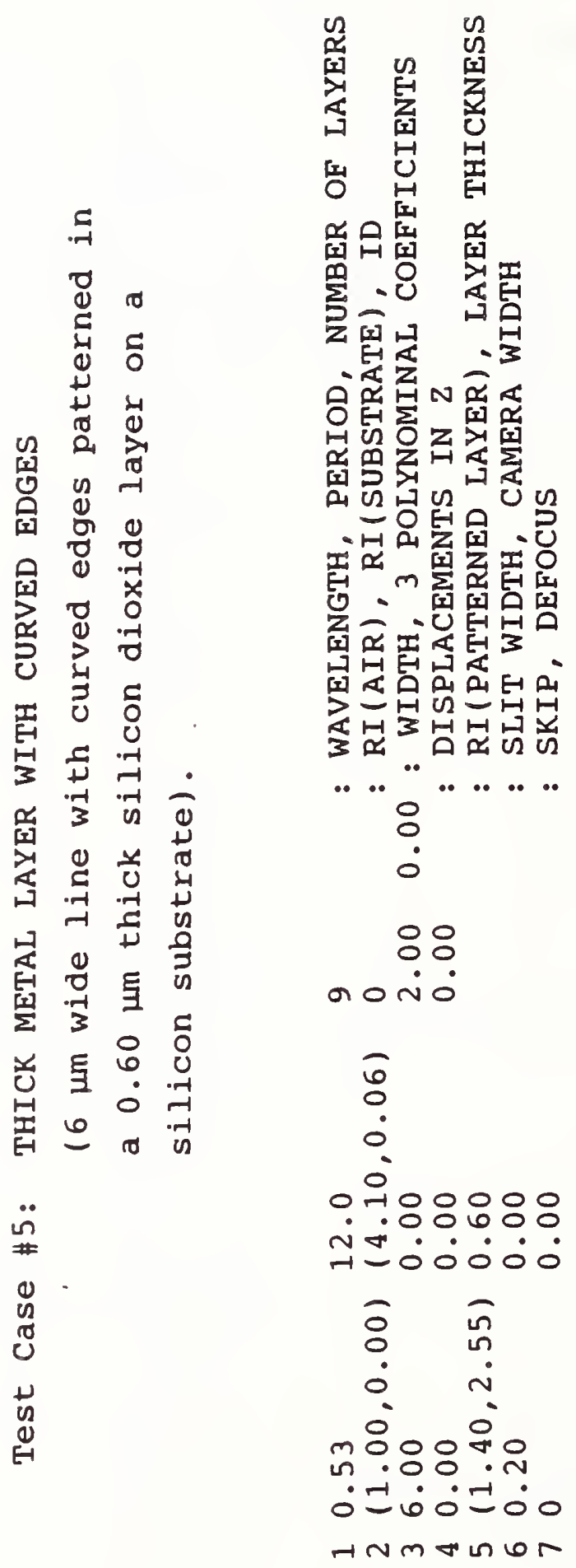


Test Case \#5: File PARFIL: Parameter File Created

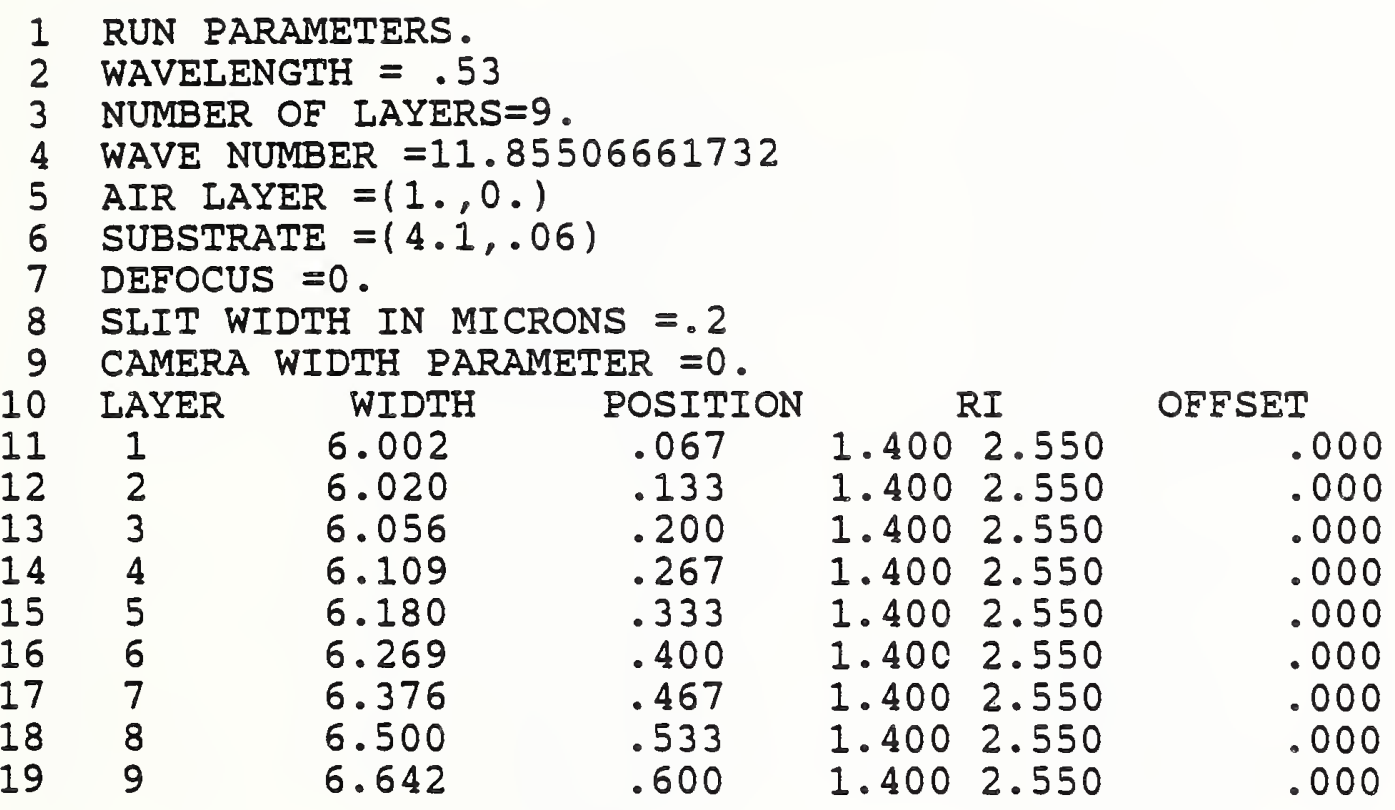


Test Case \#5: File FCOFPO: Fourier Coefficients of the Pseudo-object

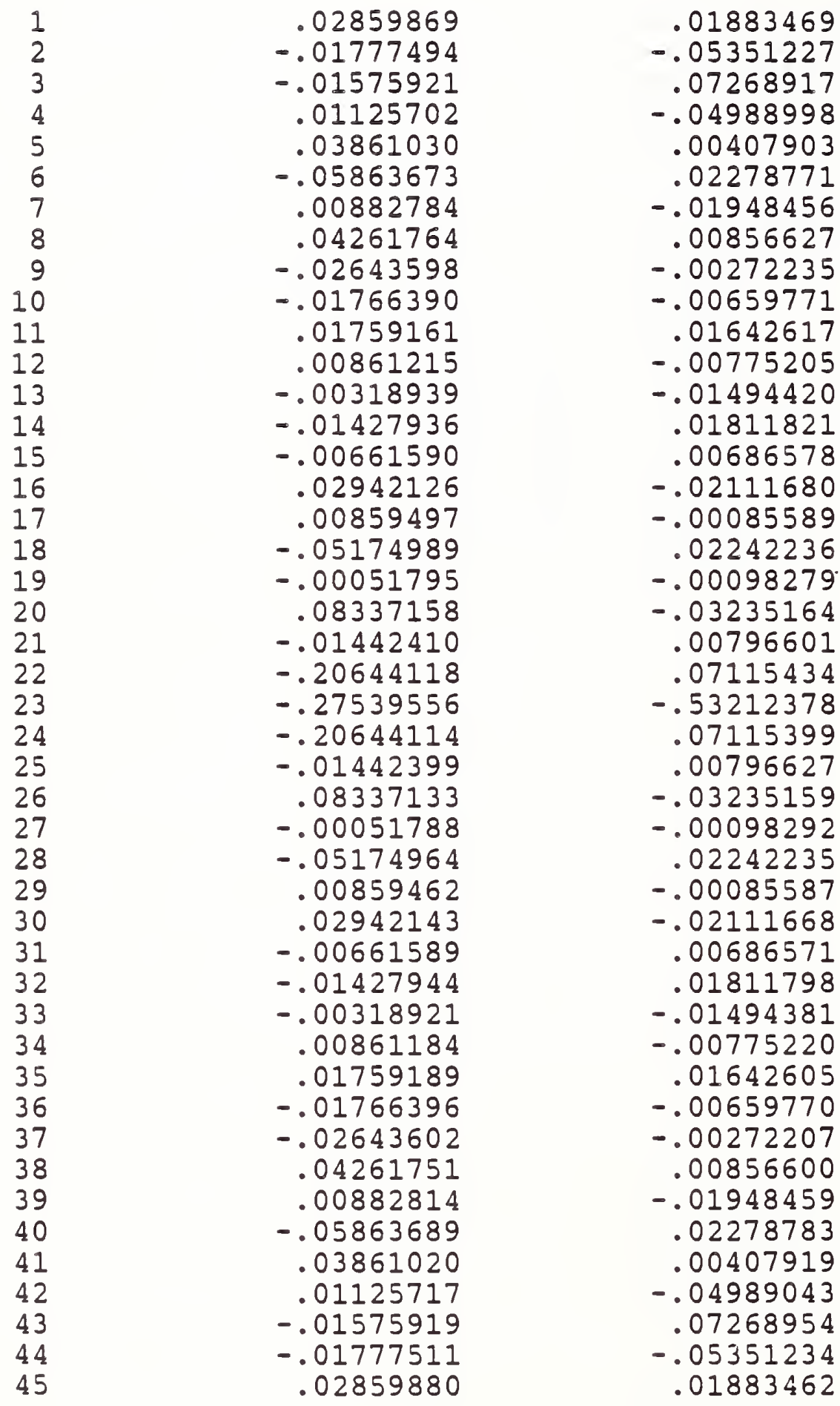




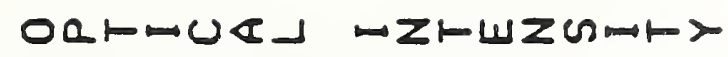

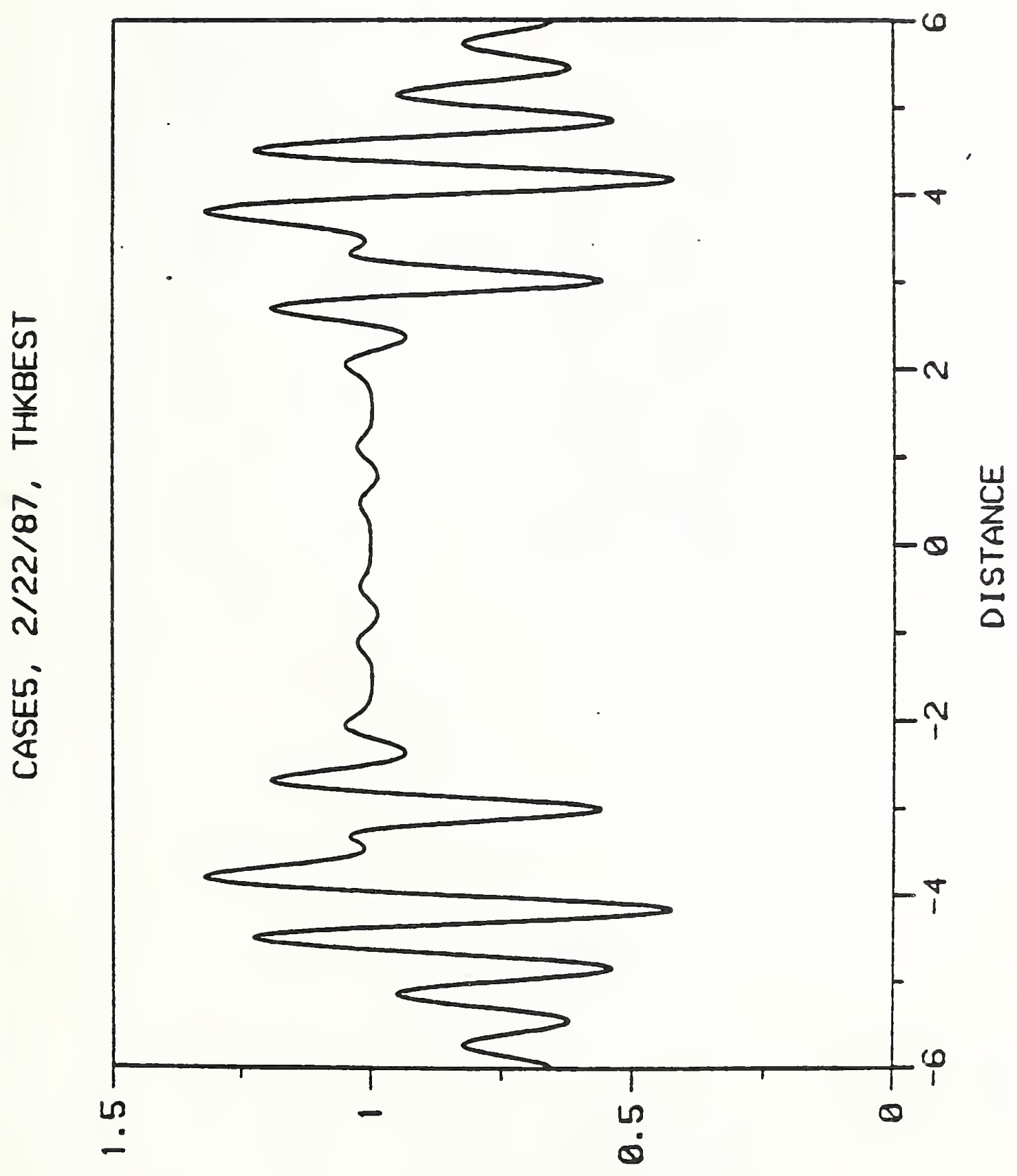

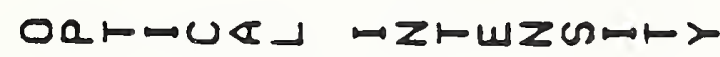

r 


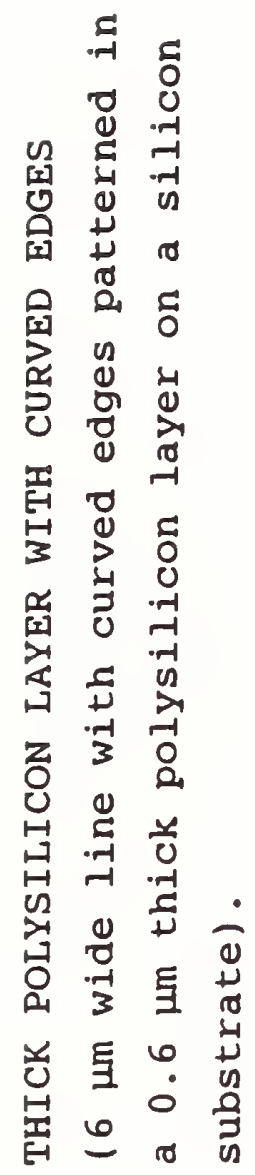

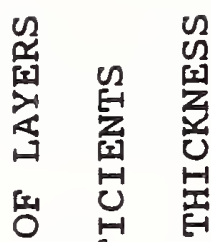

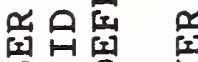

造资琵

四是

斿小的

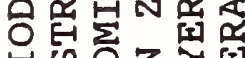

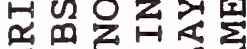

되 可

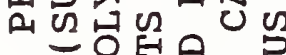
理鬼正员 텅 m $\sum_{x \rightarrow 1}$

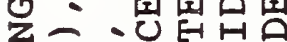

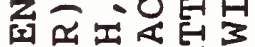

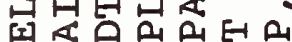

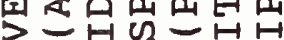

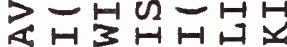

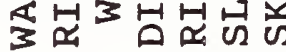<smiles>C[As](C)[O-]</smiles>

응용

a Nं०

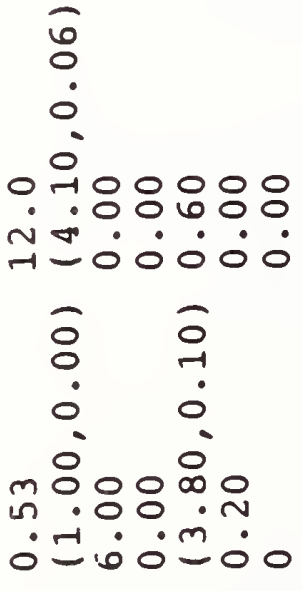

HNM 
Test Case \#6: File PARFIL: Parameter File Created

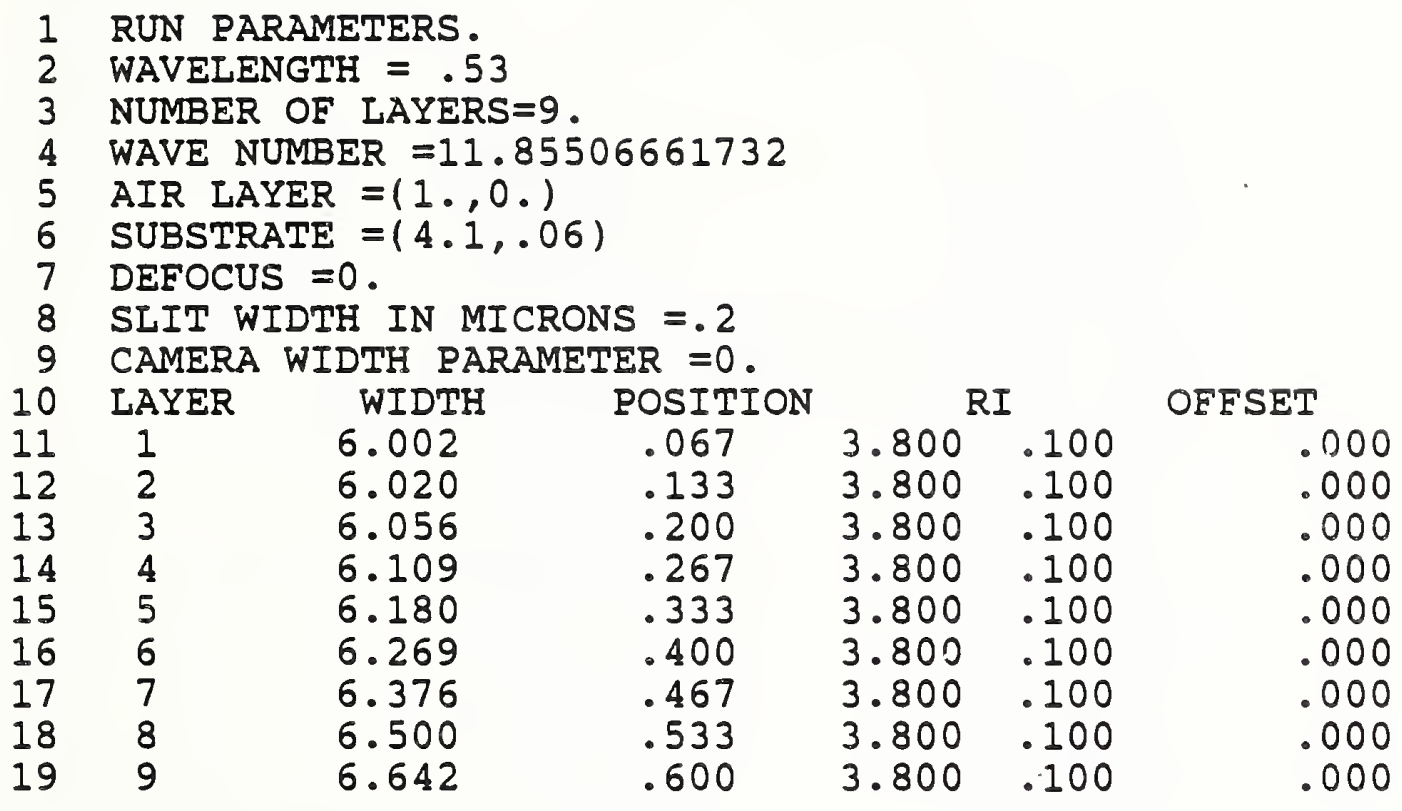


Test Case \#6: File FCOFPO: Fourier Coefficients of the Pseudo-Object

\begin{tabular}{|c|c|}
\hline $\begin{array}{r}-.06445069 \\
.00217661 \\
-.06446184 \\
-.03134388 \\
.07807896 \\
-.01812634 \\
-.06195582 \\
.04708281 \\
.03549830 \\
-.05741779 \\
-.01086065 \\
.05640716 \\
-.00874018 \\
-.04823824 \\
.02114851 \\
.03683013 \\
-.02419279 \\
-.02814956 \\
.01508793 \\
.03801847 \\
.00830037 \\
-.16673161 \\
-.31875250 \\
-.16673161 \\
.00830037 \\
.03801847 \\
.01508793 \\
-.02814956 \\
-.02419279 \\
.03683013 \\
.02114851 \\
-.04823824 \\
-.00874018 \\
.05640716 \\
-.01086065 \\
-.05741779 \\
.03549830 \\
.04708281 \\
-.06195582 \\
-.01812634 \\
.07807896 \\
-.03134388 \\
-.06446184 \\
.10217661 \\
-.06445069\end{array}$ & $\begin{array}{r}.00175338 \\
-.01417309 \\
.01696297 \\
-.00120886 \\
-.01799169 \\
.01902242 \\
-.00139914 \\
-.01378403 \\
.01048018 \\
.00419147 \\
-.01166372 \\
.00465509 \\
.00740007 \\
-.01104616 \\
.00151754 \\
.01295479 \\
-.01478962 \\
-.00686661 \\
.03343811 \\
-.01927935 \\
-.06197198 \\
.17059941 \\
-.22803972 \\
.17059941 \\
-.06197198 \\
-.01927935 \\
.03343811 \\
-.00686661 \\
-.01478962 \\
.01295479 \\
.00151754 \\
-.01104616 \\
.00740007 \\
.00465509 \\
-.01166372 \\
.00419147 \\
.01048018 \\
-.01378403 \\
-.00139914 \\
.01902242 \\
-.01799169 \\
-.00120886 \\
.01696297 \\
-.01417309 \\
.00175338\end{array}$ \\
\hline
\end{tabular}




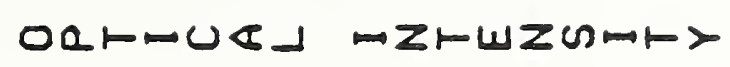

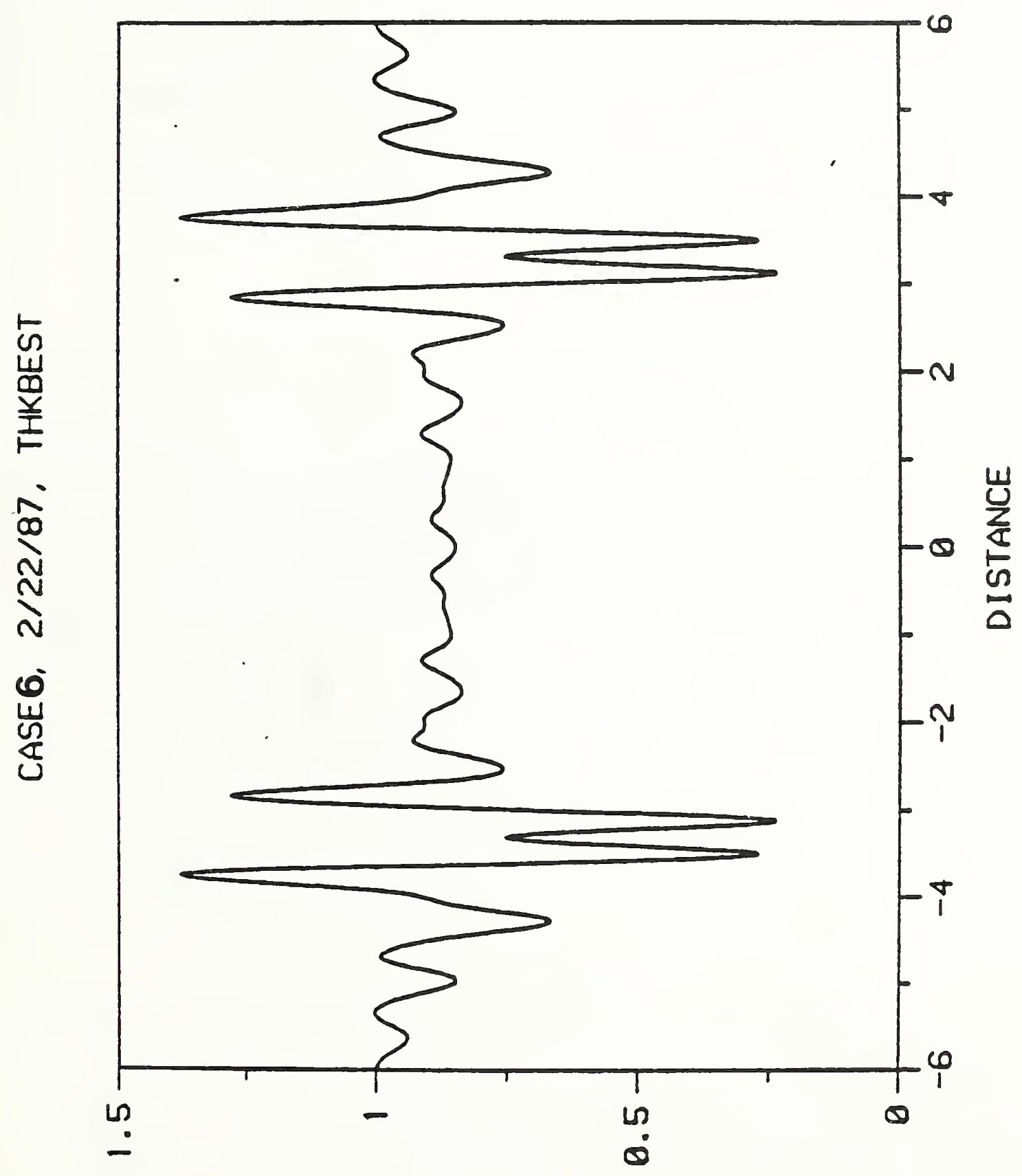

○ロトロU৫」

$r$ 


\section{Appendix III}

Reprint - "Modeling of the optical microscope image of lines patterned in thick layers with variable edge geometry," by D. Nyyssonen and C. P. Kirk (submitted to J. Opt. Soc. Am.). 
Modeling of the optical microscope imaging of lines patterned in thick layers with variable edge geometry*

$$
\text { Diana Nyyssonen** }
$$

National Bureau of Standards, Gaithersburg, MD 20899

\author{
Chris P. Kirk ${ }^{\dagger}$ \\ Vickers Instruments, Haxby Road, York. Y03 7SD. England \\ and \\ Department of Electrical and Electronic Engineering, \\ Leeds University, Leeds. IS2 9JT. England
}

\title{
Abstract
}

A monochromatic, waveguide model is presented which can predict the optical microscope images of line objects with arbitrary edge geometry, patterned in thick-layers including multilayer structures with sloping, curved, and undercut edges, granular structures such as lines patterned in polysilicon, as well as asymmetric objects. The model is used to illustrate the effects of line edge structure on the optical image. Qualitative agreement with experimentally obtained optical image profiles is demonstrated. Application of the model to study the effects of variations in layer thickness and edge geometry on linewidth measurements made at different stages of manufacturing integratedcircuit devices is discussed.

* Contribution of the National Bureau of Standards; not subject to copyright.

This work was performed under the Research Associate Agreement (CN-454) between the National Bureau of Standards and Vickers Instruments, July 1984.

** Currently with CD Metrology, Inc., Germantown, MD 20874.

+ Currently with KLA Instruments, San Jose, CA 95131. 
During the manufacture of integrated circuit wafers, critical dimensions of certain features must be measured at different stages of the production process. The control of the critical dimension of linewidth is usually monitored by using an optical or scanning-electron microscope. The measurements made by a correctly aligned microscope fitted with an appropriate measurement attachment are usually limited in accuracy and precision not by instrumental errors but by the lack of understanding of the characteristics of the image profile and the lack of accurate edge detection algorithms.

In the past, optical imaging in the microscope has been described by the scalar theory of partially coherent imaging [1-3] which characterizes the object by a planar complex transmittance or reflectance function. However, this approach does not accurately predict either the scattered field or the image structure for micrometer-sized line objects thicker than approximately onequarter of the illuminating wavelength [3]. Unfortunately, most of the line features to be measured during the production of integrated circuits fall into this category of thick objects. TYpical line objects are patterned in layers with thicknesses ranging from 0.3 to over $1.0 \mathrm{\mu m}$ (e.g. photoresist).

Integrated-circuit features encompass a wide variety of materials including dielectrics and absorbing materials of high and low conductivity (e.g., semiconductors in crystalline and amorphous states with varying amounts of dopant impurities, refractory metals, etc.) and line geometries with edge shapes varying from vertical to the complex shapes shown in Fig. 1. These lines may be etched into layers and be situated on top of metal or dielectric layers. Because of this wide variation, most of the approaches found in the literature are of only limited usefulness. 
Scattering from micrometer-sized thick objects with complex indices of refraction falls in the domain of scattering by objects whose size is comparable to the illuminating wavelength such as Mie scattering [4] and requires an electromagnetic field treatment to accurately predict the scattered field. The various approaches that have been used to treat scattering by objects on the order of a wavelength can be found in the large volume of papers primarily in the areas of scattering by particles [5], scattering by surfaces with defects or protuberances [6], or diffraction by gratings [7]. Many of these approaches are limited either to objects of specific shapes $[4,8]$ or weakly scattering objects which satisfy either the Rayleigh [9] or Born [10] approximations, or require infinite conductivity or specific grating geometries. Many are mathematically cumbersome when applied to the wide range of object shapes and materials of present interest.

Recently, the problem of imaging of line objects with vertical edge walls patterned in thick layers of dielectrics and metals was treated by Nyyssonen [3,11] who developed a model based on the waveguide analysis for thick dielectric structures of Burckhardt [12]. Burckhardt's model was originally developed for diffraction from sinusoidal dielectric gratings and was applied to the reconstruction of bleached holograms. Kaspar $[13,14]$ extended Burckhardt's approach to include absorbing photographic emulsions and non-sinusoidal grating structures and applied this approach to contact printing as well. Kaspar's work related the dielectric constant of the grating to the photographic density of the film.

The waveguide model previously used by Nyyssonen [3,11] assumes that the line structure is patterned in a nonmagnetic layer which can be characterized by its complex index of refraction which is taken to be constant with depth within the layer. Thus, this model can be used to represent homogeneous line structures with 
vertical edge walls. The spatial function representing the variation in the dielectric constant (square of the complex index of refraction) in the layer is expanded in a Fourier series. When this is substituted into the wave equation, Hill's equation [15] is obtained. The eigenvalue solutions to this equation represent waveguide modes. Assuming a single incident plane wave normal to the surface, the boundary value problem at the layer interfaces is solved to determine the Fourier coefficients in the expansions for the transmitted and reflected fields. This method allows the use of conventional scalar imaging theory to compute the image when no polarization effects are present. In such a case, the Eand H-field components are equivalent and either may be used in the scalar imaging equations.

In this paper, the waveguide model is extended to line objects whose index of refraction and geometry vary with depth in the layer, thus enabling line objects with nonvertical edges and multilayer structures to be considered. This extension of the model allows the images of virtually all line structures commonly encountered in IC fabrication to be modeled.

\section{Thick-layer Model for Variable Edge Geometry}

The method of solution for line structures of arbitrary edge geometry whose index of refraction may vary with depth in the layer is an extension of Nyyssonen's earlier method used for vertical edge walls discussed above. A line object of the type to be treated in this paper is shown in Fig. 2a. This line structure has been chosen because it contains different materials, curved edges, asymmetry, and an unpatterned sub-layer. The line structure in the patterned layer varies in both width and dielectric constant as a function of depth $z$ within the layer. Figure $2 b$ shows how this line structure may be approximated by a multilayer set of line objects each of which has a constant index of refraction over a small interval of depth $z$. The line may thus 
be represented by a set of layers with the complex dielectric constant $\varepsilon$ in each layer represented by the Fourier series expansion of:

$\varepsilon_{n}(x)= \begin{cases}\hat{n}_{n}{ }^{2}, & \left(\frac{-w_{n}}{2}+\Delta_{n}\right)<x<\left(\frac{w_{n}}{2}+\Delta_{n}\right) \\ 1, & \text { between the lines }\end{cases}$

where:

$\hat{n}_{n}=$ refractive index of the $n$th layer of the line structure, $w_{n}=$ width of the nth layer, and

$\Delta_{n}=$ offset of the nth layer in the $x$-direction. (see Fig. 2b)

Nonperiodic structures are taken as periodic with a very large repeating distance (period).

The eigenvalue solutions for the electromagnetic field components are found independently for each layer. The boundary value equations at each interface form a set of complex matrix equations which when solved allow the substitution of a single "equivalent" scattering layer for the multilayer structure. Thereafter, the solution for the transmitted and reflected field components and image are found in the same manner as for the single layer case $[3,11]$.

Following Burckhardt's method [12] and assuming monochromatic illumination where $\underline{E}=\underline{E}_{0} e^{-i \omega t}$, we start with the inhomogeneous wave equation for either the E- or H-field [16]:

$$
\begin{aligned}
& { }^{2} \underline{E}+\hat{\varepsilon}_{\mu k_{O}^{2}} \underline{E}+\operatorname{grad} \frac{\underline{E}}{\hat{\hat{\varepsilon}}} \cdot \operatorname{grad} \hat{\varepsilon}=0 \\
& { }^{2} \underline{H}+\hat{\varepsilon}_{\mu k_{O}^{2} \underline{H}}+\frac{\operatorname{grad} \hat{\varepsilon}}{\hat{\varepsilon}} \times \operatorname{curl} \underline{H}=0
\end{aligned}
$$


with $k_{0}^{2} \hat{\varepsilon} \mu=k_{0}^{2} \hat{\eta}^{2}$ for nonmagnetic media.

If we choose the $(x, y, z)$-axes as we have done so that $\hat{\varepsilon}$ is constant along the length of the line in the $y$-direction, for TE-mode $\left(\underline{E}=E_{Y} \underline{Y}\right)$ and $T M$-mode $\left(\underline{H}=H_{Y} Y\right)$ respectively, these equations simplify to

$$
\nabla^{2} E_{Y}+k_{0}^{2} \hat{\varepsilon} E_{Y}=0
$$

and

$$
\nabla^{2} H_{Y}-\frac{1}{\hat{\varepsilon}} \frac{\partial \varepsilon}{\partial X} \frac{H_{Y}}{\partial X}+k_{0}^{2} \hat{\varepsilon}_{Y}=0 . \quad \text { (TM-mode) }
$$

In the following analysis we consider only TE-mode because in the experimental NBS laser linewidth measuring system, the direction of polarization of the laser source is aligned parallel to the $y$-direction in the sample plane and the $x$-direction is the image scan direction. See Fig. 3. However, for any other system configuration and mode of illumination, the field can be considered as a superposition of $\mathrm{TE}$ - and TM-modes [17] and the corresponding analysis for TM-mode will be required. See ref. 12. Note that these modes are determined with respect to the orientation of the structure (not the incident wave) and are uncoupled (TE-mode: $E_{Y^{\prime}}$ $\mathrm{H}_{\mathrm{X}}, \mathrm{H}_{\mathrm{Z}}, \mathrm{TM}$-mode $\mathrm{H}_{\mathrm{y}^{\prime}} \mathrm{E}_{\mathrm{X}^{\prime}} \mathrm{E}_{z}$ ).

The solution for the TE-mode is taken in the form $E_{Y}(x, z)=X(x)$ $z(z)$ and since $\hat{\varepsilon}=\hat{\varepsilon}(x)$, the method of separation of variables using a separation constant a results in

$\frac{\partial^{2} X(x)}{\partial x^{2}}+k_{0}^{2} \hat{\varepsilon}(x) x(x)+a^{2} x(x)=0$ 
$\frac{\partial^{2} z}{\partial z^{2}}=a^{2} z(z)$

These equations hold for any functional form of $\hat{\varepsilon}(x)$. However, following Burckhardt, we choose the Fourier approach where $\hat{\varepsilon}_{n}(x)$ from eq. (1) is represented by the Fourier series

$\hat{\varepsilon}_{n}(x)=\sum_{q} E_{q, n} \exp (2 \pi i q x / P)$

where

$E_{q, n}= \begin{cases}\left(w_{n} / P\right) \hat{n}_{n}^{2}+\left(1-w_{n} / P\right) \hat{n}_{0}^{2} & q=0 \\ \frac{\left(\hat{n}_{n}^{2}-\hat{n}_{0}^{2}\right)}{q \pi} \exp \left(2 \pi i \Delta_{n} q / P\right) \sin \left(q \pi w_{n} / P\right) & q \neq 0\end{cases}$

where $\mathrm{P}$ is the period chosen arbitrarily large when the object consists of a single line and $q$ is the summation index. Substituting eq. (8) into eq. (6) yields the differential equation known as Hill's equation

$\frac{\partial^{2} x(x)}{\partial x^{2}}+k_{0}^{2}\left[\sum_{q} E_{q, n} \exp (2 \pi i q x / P)\right] x(x)+a^{2} x(x)=0$

The solution for this general form of Hill's equation is given by Kaspar and others $[13,15]$. The form of the eigenvalue matrix is given in the Appendix. The solution of eq. (9) for the $n$-th layer is of the form

$$
\begin{aligned}
E_{\dot{y}}^{n}(x, z)= & \sum_{m}\left[A_{m, n} \exp \left(\alpha_{m, n} z\right)+A_{m, n}^{\prime} \exp \left(-\alpha_{m, n} z\right)\right] \\
& \cdot \sum_{j} B_{j, m, n} \exp (2 \pi i j x / P)
\end{aligned}
$$


where the $a_{m, n}$ 's are the eigenvalues and the $B_{j, m, n}$ 's are the eigenvector solutions to Hill's equation. The $A_{m, n}$ and $A_{m, n}^{\prime}$ are weighting constants which must be determined from the boundary conditions. Each of these terms represents an inhomogeneous plane wave or waveguide mode which is supported by the line structure. Note that, when there is no absorption, ( $\hat{n}$ real) the eigenvalues are purely imaginary and this form reduces to that of Burckhardt.

The solution for the transmitted and reflected fields is found by equating the tangential components of the E- and H-fields at each layer interface. The $x$-component of the H-field is found from ik $\mathrm{H}_{\mathrm{H}}=$ curl $\underline{E}$ or, here, $i k_{0} \mathrm{H}_{\mathrm{x}}=-\partial E_{\mathrm{y}} / \partial z$ :

$$
\begin{aligned}
H_{x}^{n}(x, z)= & \sum_{m}\left[A_{m, n}\left(\frac{a_{m, n}}{-i k_{0}}\right) \exp \left(\alpha_{m, n} z\right)-A_{m, n}^{\prime}\left(\frac{\alpha_{m, n}}{-i k_{0}}\right) \exp \left(-\alpha_{m, n} z\right)\right] \\
& \cdot \sum_{j} B_{j, m, n} \exp (2 \pi i j x / P) .
\end{aligned}
$$

With the tangential components of the incident field given by:

$E_{Y}^{I}=E_{0}^{I} \exp \left(i k_{0} z\right)$

$H_{x}^{I}=-E_{0}^{I} \exp \left(i k_{0} z\right)$

the tangential components of the reflected field by:

$$
E_{y}^{R}(x, z)=\sum_{j} E_{j}^{R} \exp \left\{-i k_{0}\left[\left(\frac{\lambda j}{P}\right) x+k_{j}^{R}\right]\right\}
$$


$H_{x}^{R}(x, z)=\sum_{j} k_{j} E_{j}^{R} \exp \left\{-i k_{0}\left[\left(\frac{\lambda j}{P}\right) x+k_{j}^{R}\right]\right\}$,

where $k_{j}^{R}=\sqrt{1-\left(\frac{\lambda j}{P}\right)^{2}}$

and the tangential components of the transmitted field by:

$E_{Y}^{T}(x, z)=\sum_{j} E_{j}^{T} \exp \left\{i k_{0}\left[\left(\frac{\lambda j}{P}\right) x+k_{j}^{T} z\right]\right\}$

$\mathrm{H}_{x}^{\mathrm{T}}(x, z)=\sum_{j}-\mathrm{K}_{j}^{\mathrm{T}} \mathrm{E}_{j}^{\mathrm{T}} \exp \left\{i k_{\circ}\left[\left(\frac{\lambda j}{P}\right) x+\mathrm{K}_{j}^{\mathrm{T}} z\right]\right\}$

where $k_{j}^{T}=\sqrt{\hat{n}_{s}{ }^{2}-\left(\frac{\lambda j}{P}\right)^{2}}$ and $\hat{n}_{s}$ is the complex index of the substrate.

For each Fourier component $j$, the following boundary equations must be satisfied:

1. At $\underline{z=0}(x=y=0)$, the solution must match the tangential components of the $\underline{E}-$ and $\underline{H}-f i e l d s$ at the top surface:

$E_{o}^{I} \delta_{j 0}+E_{j}^{R}=\sum_{m}\left[A_{m, 1}+A_{m, 1}^{\prime}\right] B_{j, m, 1} ;$

$-E_{0}^{I} \delta_{j 0}+k_{j}^{R} E_{j}^{R}=\sum_{m}\left[A_{m, 1}\left(\frac{a_{m, 1}}{-i k_{0}}\right)-A_{m, 1}^{\prime}\left(\frac{a_{m, 1}}{-i k_{0}}\right)\right] B_{j ; m, 1}$,

where $\delta_{j 0}= \begin{cases}1 & j=0 \\ 0 & j \neq 0\end{cases}$ 
2. At the interface between the $n$-th and $(n+1)-t h$ layer, $z=-z_{n}(x=y=0)$, the $\underline{E}-$ and H-fields must satisfy the boundary conditions at each interface:

$$
\begin{gathered}
\sum_{m}\left[A_{m, n} \exp \left(a_{m, n} z_{n}\right)+A_{m, n}^{\prime} \exp \left(-a_{m, n} z_{n}\right)\right] B_{j, m, n} \\
=\sum_{m}\left[A_{m, n+1} \exp \left(a_{m, n+1 n}\right)+A_{m, n, 1}^{\prime} \exp \left(-a_{m, n+1} z_{n}\right)\right] B_{j, m, n+1} \\
\sum_{m}\left[A_{m, n}\left(\frac{a_{m, n}}{-i k_{0}}\right) \exp \left(a_{m, n} z_{n}\right)-A_{m, n}^{\prime}\left(\frac{a_{m, n}}{-i k_{0}}\right) \exp \left(-a_{m, n} z_{n}\right)\right] B_{j, m, n} \\
=\sum_{m}\left[A_{m, n+1}\left(\frac{a_{m, n+1}}{-i k_{0}}\right) \exp \left(a_{m, n+1} z_{n}\right)\right. \\
\left.-A_{m, n+1}^{\prime}\left(\frac{a_{m, n+1}}{-i k_{0}}\right) \exp \left(-a_{m, n+1} z_{n}\right)\right] B_{j, m, n+1} .
\end{gathered}
$$

3. At $z=-z_{N}=-T(x=y=0)$, the solution must satisfy the boundary conditions imposed by the substrate (assumed infinite in extent - see Fig. 3):

$$
\begin{gathered}
\sum_{m}\left[A_{m, N} \exp \left(a_{m, N} T\right)+A_{m, N}^{\prime} \exp \left(-a_{m, N} T\right)\right] B_{j, m, N} \\
=E_{j}^{T} \exp \left(-i k_{0} K_{j}^{T} T\right) \\
\sum_{m}\left[A_{m, N}\left(\frac{a_{m, N}}{-i k_{0}}\right) \exp \left(-\alpha_{m, N} T\right)-A_{m, N}^{\prime}\left(\frac{a_{m, N}}{-i k_{0}}\right) \exp \left(a_{m, N} T\right)\right] B_{j, m, N} \\
=-K_{j}^{T} E_{j}^{T} \exp \left(-i k_{0} K_{j}^{T}\right) .
\end{gathered}
$$


In matrix notation,

$$
\begin{aligned}
& \left(\begin{array}{c}
E_{X}^{I R} \\
H_{Y}^{I R}
\end{array}\right)=\left(B_{1}(0)\right) \cdot\left(\begin{array}{l}
A_{1} \\
A_{1}^{\prime}
\end{array}\right) \\
& \left(B_{n}\left(Z_{n}\right)\right) \cdot\left(\begin{array}{l}
A_{n} \\
A_{n}^{\prime}
\end{array}\right)=\left(B_{n+1}\left(Z_{n}^{\prime}\right) \cdot\left(\begin{array}{l}
A_{n+1} \\
A_{n+1}^{\prime}
\end{array}\right)\right. \\
& \left(B_{N}(T)\right) \cdot\left(\begin{array}{c}
A_{N} \\
A_{N}^{\prime}
\end{array}\right)=\left(\begin{array}{c}
E_{X}^{T} \\
H_{Y}^{T}
\end{array}\right)
\end{aligned}
$$

where

$$
B_{n}\left(z_{n}\right)=\left(\begin{array}{ll}
B_{n}^{11}\left(z_{n}\right) & B_{n}^{12}\left(z_{n}\right) \\
B_{n}^{21}\left(z_{n}\right) & B_{n}^{22}\left(z_{n}\right)
\end{array}\right)
$$

and the matrix elements are given by

$$
B_{j, m, n}^{11}\left(z_{n}\right)=\exp \left(-a_{m, n} z_{n}\right) B_{j, m, n}
$$

$$
B_{j, m, n}^{12}\left(z_{n}\right)=\exp \left(\alpha_{m, n} z_{n}\right) B_{j, m, n}
$$$$
B_{j, m, n}^{21}\left(z_{n}\right)=\left(\frac{a_{m, n}}{-i k_{0}}\right) \exp \left(-a_{m, n} z_{n}\right) B_{j, m, n}
$$$$
B_{j, m, n}^{22}\left(z_{n}\right)=-\left(\frac{a_{m, n}}{-i k_{0}}\right) \exp \left(a_{m, n} z_{n}\right) B_{j, m, n}
$$ 
Depending upon whether the solution for the transmitted or reflected field is desired, all of the $A_{n}$ and $A_{n}^{\prime}$ s are eliminated from these equations except for either $A_{N}$ and $A_{N}^{\prime}$ or $A_{1}$ and $A_{1}, i . e .$, for the transmitted field:

$$
\begin{aligned}
\left(\begin{array}{l}
A_{1} \\
A_{1}^{\prime}
\end{array}\right)=\left(B_{1}\left(z_{1}\right)\right)^{-1} \cdot\left(B_{2}\left(z_{1}\right)\right) \cdot\left(B_{2}\left(z_{2}\right)\right)^{-1} \ldots \\
\cdot\left(B_{N-1}\left(z_{N-1}\right)\right)^{-1} \cdot\left(B_{N}\left(z_{N-1}\right)\right) \cdot\left(\begin{array}{l}
A_{N} \\
A_{N}^{\prime}
\end{array}\right)=B \cdot\left(\begin{array}{l}
A_{N} \\
A_{N}^{\prime}
\end{array}\right) .
\end{aligned}
$$

or, for the reflected field:

$$
\begin{aligned}
\left(\begin{array}{l}
A_{N} \\
A_{N}^{\prime}
\end{array}\right)=\left(B_{N}\left(z_{N-1}\right)\right)^{-1} \cdot\left(B_{N-1}\left(z_{N-1}\right)\right) \cdot\left(B_{N-1}\left(z_{N-2}\right)\right)^{-1} \ldots \\
\cdot\left(B_{2}\left(z_{1}\right)\right)^{-1} \cdot\left(B_{1}\left(z_{1}\right)\right) \cdot\left(\begin{array}{c}
A_{1} \\
A_{1}
\end{array}\right)=B^{-1} \cdot\left(\begin{array}{l}
A_{1} \\
A_{1}
\end{array}\right)
\end{aligned}
$$

This operation replaces the multilayer structure with an equivalent layer characterized by the matrix $B$.

The equations are now solved as for the single layer case by eliminating the unknown $E_{j}^{R}$ and $E_{j}^{T}$ to solve for the $A_{1} s$ or $A_{N} s$ again depending upon whether the Fourier expansion of the reflected or transmitted field is desired. In the present paper, we compute the reflected field coefficients,

$$
\left(\begin{array}{c}
E_{X}^{I R} \\
H_{Y}^{I R}
\end{array}\right)=\left(B_{1}(0)\right) \cdot\left(\begin{array}{c}
A_{1} \\
A_{1}^{\prime}
\end{array}\right)
$$


$\left(\begin{array}{ll}B^{11} & B^{12} \\ B^{21} & B^{22}\end{array}\right) \cdot\left(\begin{array}{l}A_{1} \\ A_{1}\end{array}\right)=\left(\begin{array}{c}E_{X}^{T} \\ H_{Y}^{T}\end{array}\right)$

where

$\left(\begin{array}{ll}B^{11} & B^{12} \\ B^{21} & B^{22}\end{array}\right)=\left(B_{N}(T)\right) \cdot B^{-1}$

Using the format of eqs. (18) this reduces to the single matrix equation:

$\left(\begin{array}{cc}D_{11} & D_{12} \\ D_{21} & D_{22}\end{array}\right) \cdot\left(\begin{array}{c}A_{1} \\ A_{1}\end{array}\right)=\left(\begin{array}{l}R \\ 0\end{array}\right)$

where

$D_{j, m}^{11}=\left[k_{j}^{R}-\left(\frac{a_{m, 1}}{-i k_{0}}\right)\right] B_{j, m, 1}$

$D_{j, m}^{12}=\left[k_{j}^{R}+\left(\frac{a_{m, 1}}{-i k_{0}}\right)\right] B_{j, m, 1}$

$D_{j, m}^{21}=\left[k_{j}^{T} B_{11}+B_{21}\right]$

$D_{j, m}^{22}=\left[k_{j}^{T} B_{12}+B_{22}\right]$

and

$R_{j}=\left[E_{0}^{I}\left(1+K_{j}^{R}\right) \delta_{j 0}\right]$

where the desired coefficients $E_{j}^{R}$ are found from eq. (15a) 
$E_{j}^{R}=\sum_{m}\left[A_{m, 1}+A_{m, 1}^{\prime}\right] B_{j, m, 1}-E_{0}^{I} \delta_{j 0}$

These coefficients represent the Fourier coefficients of a pseudo or equivalent (planar) object. The microscope image of the reflected field is then computed using scalar imaging theory (see Ref. 3.) with the line object reflectance function given by

$t(x)=\sum_{j} E_{j}^{R} \exp \left(i k_{0} \lambda j x / P\right)$

This method of computing both the reflected field and the corresponding microscope image requires no approximations of the type usually found, such as limits on the conductivity or slope of the surface, etc. Iimitations may be imposed, however, by the computation capability available. First, the number of layers used to approximate the structure increases the computing time linearly. In most of the cases to be shown here, seven to nine layers were sufficient to produce significant results and required approximately one minute of CPU time on a Univac 1108.

The second limitation is in the truncation of the series, i.e., the matrix sizes used in the computations. In the present case, as for a single layer [3], all of the reflected plane waves are included which have diffraction angles less than $\pm \pi / 2$ in air. With $P=12 \mu \mathrm{m}$ and $\lambda=0.53 \mu \mathrm{m}, 22$ diffracted orders are included which requires a $45 \times 45$ complex eigenvalue matrix and a $90 \times 90$

\footnotetext{
*Certain commercial equipment, instruments, or materials are identified in this paper in order to adequately specify experimental or computational procedure. Such identification does not imply recommendation or endorsement by the National Bureau of standards, nor does it imply that the materials or equipment identified are necessarily the best available for the purpose.
} 
complex matrix for inversion of the $B$ matrices in eqs. (19). This choice necessarily truncates the series which represents the field in the layers with higher refractive index. In the cases considered here, this truncation does not appear to significantly affect the results.

Also, for grating objects with $\mathrm{P} \leq 12 \mu \mathrm{m}$, the computations are exact. However, for isolated line objects near resonances (where the thickness times index of refraction of the patterned layer is approximately equal to the wavelength), $\mathrm{P}=12 \mu \mathrm{m}$ is not large enough to eliminate the effect of the adjacent lines on the image. Larger matrix sizes would have to be used where $P>12$ um is required.

The Effect of Geometry on the Image Profile

The present method was developed primarily for the purpose of modeling the effect of nonvertical edges on the image profile. There is a very wide range of edge geometries which can be modeled, therefore, only a few key examples will be presented here in order to demonstrate trends. Figure 1 shows six basic shapes which are frequently encountered in integrated circuit processing. In order to simplify the definition of these shapes, they will be represented by a polynominal expansion which defines the width as a function of $z$.

$w(z)=\sum_{j=0}^{J} x_{j}\left(z-z_{j}\right)^{j}$,

where $\mathrm{J}=$ the polynominal order,

$$
\begin{aligned}
& x_{j}=\text { coefficient of the } j \text {-th order, and } \\
& z_{j}=\text { offset of the } j \text {-th order. }
\end{aligned}
$$


The shapes in Fig. 1 have been restricted to fifth order polynominals $(J \leq 5)$. In these examples it will be assumed that the patterned layer is $0.6 \mathrm{\mu m}$ thick, and the nominal linewidth $x_{0}$ is 6.0 $\mu m$. The line consists of loss-free silicon dioxide with a real refractive index of 1.46 . The substrate is taken to be silicon with a complex refractive index of $4.1+0.06 i$ at an illumination wavelength of $0.53 \mu \mathrm{m}$. The diffraction-limited images have been computed for a 0.14 NA illumination aperture and 0.85 NA objective aperture. Note that in this polynominal representation of the line object, although $x_{0}$ as shown in Fig. 1 is the nominal linewidth, $x_{0}$ is: the width at the top $(z=0)$ in cases $(b)$ and $(g)$; the width at the bottom $(z=d)$ in cases (c), (d), and (f), and the mean width in cases (e) and (h). Figure 4 shows the theoretical image intensity profile of an ideal line with vertical edge walls. The oxide line extends from -3.0 to $+3.0 \mu \mathrm{m}$ as shown by the dashed lines, and the rest of the object is bare silicon. In the calculation of the Fourier series coefficients, this structure is assumed to be repeated with a period of $12.0 \mathrm{\mu m}$. The line edge image is characterized by a dark fringe wider than the interference fringe that would be calculated for a similar thin layer.

The set of image profiles in Fig. 5 demonstrates the effect of different edge geometries on the image. The line has been assumed to be symmetrical and so only half the image profile is shown. The line objects are defined in terms of Eq. (2) and the width of the line edge is getting progressively broader down each of the three columns. There are two effects which are common to all three geometries. As the edge becomes broader, the dark fringe associated with the edge also becomes broader with the bright fringes on either side becoming brighter than occurs with partially coherent imaging of planar objects. When the edge becomes very broad, the dark fringe itself begins to broaden out and small peaks form within it. 
According to the method described so far, the object is assumed to consist of homogeneous slabs of materials. This assumption is implicit in Eq. (1). In practice many materials such as polycrystalline silicon cannot be regarded as homogeneous as they have a definite internal structure which will result in a variation in refractive index within the material. These structures can be modeled by representing the random refractive index disturbances within the material by functions.

$$
\hat{\varepsilon}_{n}^{\prime}(x)=\hat{\varepsilon}_{n}(x)+\sum_{j=1}^{J} c_{j} \delta\left(x-x_{j}\right),
$$

where:

$$
\begin{aligned}
& \hat{\varepsilon}_{n}(x)=\text { refractive index profile from } \mathrm{Eq} .(1), \\
& c_{j}=\text { amplitude of } j-t h \text { refractive index disturbance, and } \\
& x_{j}=\text { position of } j-t h \text { disturbance. }
\end{aligned}
$$

The function $\hat{\varepsilon}_{n}(x)$ now describes a noisy layer, and by restricting the range of values for $x_{j}$, the noise may be confined to different parts of the layer.

Figure 6 shows three image profiles for a noisy structure. The object is the oxide structure used to produce the profile in Fig. 4 and noise has been added to the entire layer. In each of the three cases shown in Fig. 6, different sets of noise data have been used corresponding to different sets of $C_{j}$ selected from a random number table. The most striking feature of these curves is that, although the field is perturbed within the area of the object, the dark fringe at the edge of the line remains well defined, and relatively unperturbed. 
In order to test the model against experimental image profiles, a test specimen was prepared which consisted of lines patterned in photoresist on a silicon substrate. The same test pattern was put down repeatedly by projection printing but with a range of focus positions. This produced a series of patterns with a range of edge slopes. Two patterns were selected to illustrate different edge properties. One pattern corresponded to the in-focus exposure and had near vertical edge walls; the second corresponded to a considerable defocus on exposure and had significantly sloping edges.

The in-focus exposure was assumed to produce near vertical edge walls, and when the structures were examined in a scanning electron microscope, this was found to be the case. The edge walls had slopes of about $80^{\circ}$, which according to the theory may effectively be assumed to be vertical for films of submicrometer thickness. The width of the window in the resist was taken as $2.5 \mathrm{\mu m}$.

The out-of-focus pattern had significant edge slopes, and the structure was modeled by the asymmetric third order window shown in Fig. 7, based on SEM pictures of the cross section. The photoresist thickness was not known exactly but was nominally $1 \mathrm{um}$. The refractive index of the resist is not known exactly, but this is not serious as small changes in the refractive index can be offset by small adjustments in the layer thickness without significantly changing the image profile. The refractive index of the photoresist was assumed to be 1.513. The theoretical layer thickness was adjusted until it produced the same contrast as the experimental data; this gave a thickness for the resist of $0.94 \mathrm{um}$.

It is very difficult to accurately determine the position corresponding to "best focus" when viewing these thick structures in 
the optical linewidth measurement system. Therefore, it is necessary to generate a series of profiles as a function of focus position and compare these with the theoretical data. Figure 8 shows a comparison of the experimental and theoretical image profiles produced by these structures. The experimental data were generated using the coherent optical linewidth measurement system which has been described in detail in the literature by Nyyssonen. [3]

The profiles for the sloping edge structure are quite different from those for the vertical edge structure. However, in both cases they show good agreement with the models. The theoretical profiles display the same features as the experimental profiles, but the agreement is not perfect. One significant source of differences is the assumption that the resist is homogeneous. This is not strictly true. To get better agreement, it would have been advantageous to use a material such as silicon dioxide which is homogeneous and which would produce a stable and accurately known refractive index.

A second. source of differences may arise from the assumption that the illumination is normally incident. As shown in the experimental data, the central peak of the sloping edge profile moves as the object moves through focus and the line with nearly vertical edges generates slightly asymmetric profiles (see Fig. 8). Both of these may be attributed to slight asymmetry in either the illumination system or imaging optics. The NBS profiling system used to generate these profiles was checked for illumination symmetry when profiling thin layers. However, it is expected that these thick layers are significantly more sensitive to asymmetric illumination than is a thin-layer object.

Case Study of the Production of an Mos Transistor

This section considers some typical structures which are encountered in microlithography and the effect of small variations in 
these structures on the optical image profile. The two structures shown in Fig. 9 are considered. These structures represent the key stages of patterning the polysilicon layer when making an Mos device. The structure in Fig. $9($ a) represents the stage between patterning the resist and etching the polysilicon. Controlling the resist dimensions at this stage will help control the final etched polysilicon linewidth. However, measuring the width of the resist line at this stage is difficult because the image profile is a function of the geometry, refractive index, and thickness of each of the three layers. The polysilicon was assumed to have a refractive index of $3.8+0.1 i$.

The curves in Fig. 10 show how variations in the thickness of the polysilicon affect the image profile of the resist line. Again, only half the image profile is shown. The most striking feature of these curves is that the image structure in the immediate vicinity of the line edge is sensitive to the thickness of the polysilicon layer.

The structure in Fig. 11 represents a polysilicon line after etching and stripping of the resist. The image profile is a function of the thickness of the silicon-dioxide layer. Figure 12 shows the effect of changing the thickness of the oxide sublayer on the image profile of the structure shown in Fig. 9(b). The polysilicon is assumed in this case to have vertical edge walls and the oxide thickness is varied over a range of $40 \mathrm{~nm}$. As the thickness changes, the image profile changes considerably. This makes linewidth measurement difficult as there appears to be no feature or threshold which locates the edge independently of oxide thickness.

The image profile is also a function of edge geometry and in Fig. 9 (b) it has been assumed that the edge shape can be defined by a second order polynomial with the polynomial of Eq. (2) given by 
$w(z)=x_{0}+x_{2} \cdot z^{2}$

where, again, the nominal linewidth $x_{0}$ is $6.0 \mu \mathrm{m}$. The curves in Fig. 12 show the effect of variations in the edge curvature of the polysilicon layer on the image profile. The edge image profile is clearly sensitive to the curvature of the physical edge of a curved feature. In this case, three possible definitions of linewidth are the minimum, maximum, or mean width of the layer. For the examples shown in Fig. 12, these definitions all give linewidths which do not correspond to that given by the position of the minimum of the dark fringe. More importantly, however, the offset between the true and measured linewidth varies with the degree of curvature over a range of a few tenths of a micrometer regardless of the definition used for the line edge.

\section{Summary}

A waveguide model has been presented which enables the optical images of line structures patterned in thick layers to be computed. The model has been shown to be applicable to a wide range of structures, and qualitative agreement with experimental image profiles has been demonstrated. From the results presented here, it can be seen that the image profile of a thick line object, and therefore the measured linewidth, is affected by the physical shape of the edges.

The model enables the effects of process variations on the optical image profile to be determined and this, in turn, enables the accuracy and sensitivity of different measurement techniques to be investigated. Further work is in progress to improve existing measurement methods in order to minimize the effects of process variations on the accuracy and repeatability of linewidth measurements. 


\section{Acknowledgments}

The authors wish to acknowledge the help of Marilyn J. Dodge who collected the experimental image profiles for this work. This work was carried out as part of one of the author's (Kirk) Ph.D. research program supported by the Science and Engineering Research Council (UK). 


\section{Appendix}

In matrix notation, the eigenvalue problem of eq. (9) is:

$D \cdot B_{n}=a^{2} I \cdot B_{n}$

where the matrix elements $D_{i, j}$ for the $n$-th layer are given by

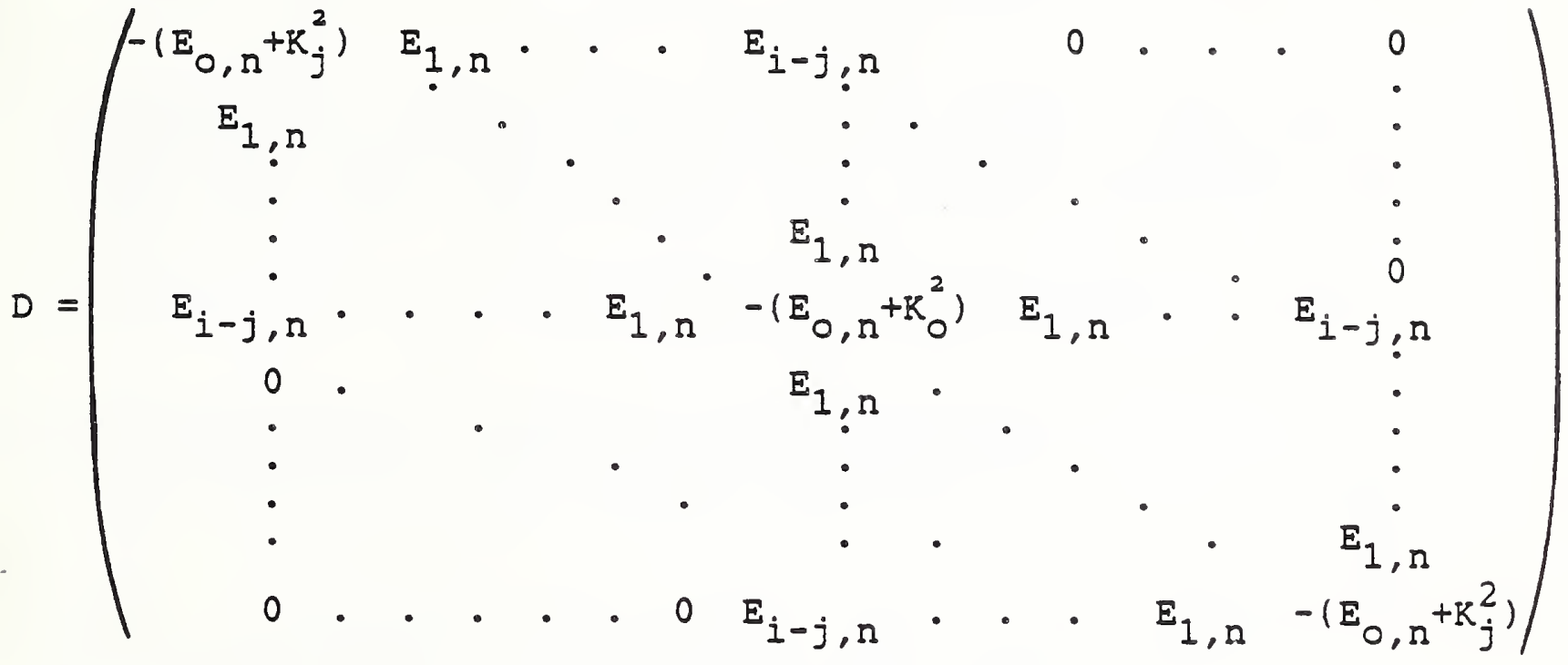

and $k_{j}=\frac{\lambda j}{P}$ 
1. M. Born and E. Wolf, Principles of Optics, 5 th ed., (Pergamon Press, Oxford, 1975), pp. 522-532.

2. L. C. Martin, The Theory of the Microscope, (Blackie, London, 1966), Chapters V and VIII.

3. D. Nyyssonen, "Theory of optical edge detection and imaging of thick layers", J. Opt. Soc. Am. 72, pp. 1425-1436 (1982).

4. Ref. 1, Section 13.5, pp. 633-664.

5. P. W. Barber, D. Y. Wang, and M. B. Long, "Scattering calculations using a microcomputer," App. Opt. 20, 1121-1123 (1981).

6. J. P. Hugonin and R. Petit, "Theoretical and numerical study of a locally deformed stratified media," J. Opt. Soc. Am. 71, 664-674 (1981).

7. R. Petit, ed., Electromagnetic Theory of Gratings, (SpringerVerlag, Berlin, 1980).

8. K. A. Zaki and A. R. Neureuther, "Scattering from a perfectIy conducting surface with sinusoidal height profile, TE polarization," IEEE Trans. Antennas. Propagat. AP-19, 208214 (1971).

9. J. P. Hugonin, R. Petit, and M. Cadilhac, "Plane-wave expansions used to describe the field diffracted by a grating," J. Opt. Soc. Am. 71, 593-598 (1981).

10. Ref. 1, p. 453. 
11.

D. Nyyssonen, "Optical linewidth measurement on patterned metal layers," Proc. SPIE 480 , Integrated Circuit Metrology II, 65-70 (1984).

12. C. B. Burckhardt, "Diffraction of a plane wave at a sindsoidally stratified dielectric grating", J. Opt. Soc. Am. 56, pp. 1502-1509 (1966).

13. F. G. Kaspar, "Diffraction by thick, periodically stratified gratings with complex dielectric constant," J. Opt. Soc. Am. 63, 37-45 (1973). 14. F. G. Kaspar, "Computation of light transmitted by a thick grating, for application to contact printing," J. Opt. Soc. Am. 64, 1623-1630 (1974).

15. W. Magnus and S. Winkles, Hill's Equation (John Wiley and Sons, New York, 1966 and Dover, New York, 1979).

16. Ref. 1, p. 612.

17. Ref. 1, p. 579.

77 

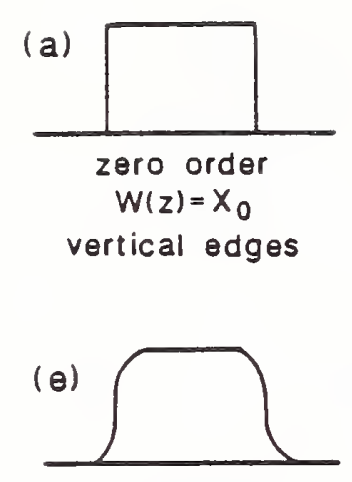

third order

$W(z)=x_{0}+x_{3}(z-d / 2)^{3}$ baked resis? etched metal
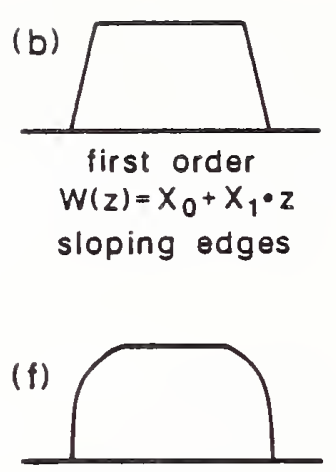

fourth order $W(z)=X_{0}-X_{4}(z-d)^{4}$ baked resist
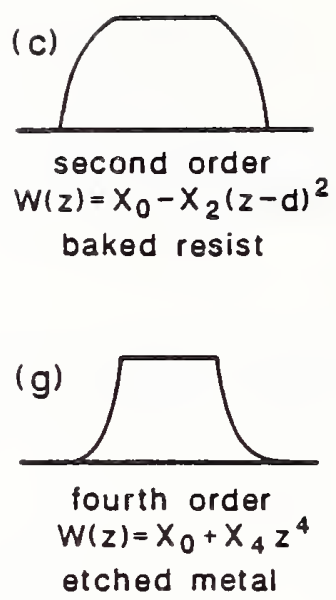

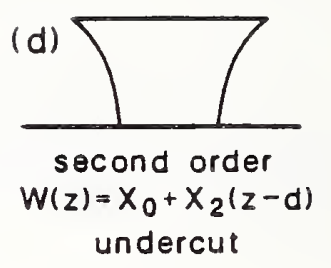

(h)

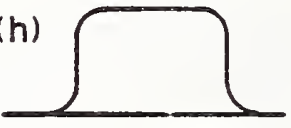

fifth order

$W(z)=X_{0}+X^{2}(z-d / 2)^{5}$ baked resist

Fig. 1. The geometry of most line objects encountered in integrated circuits may be approximated by low order polynomials.

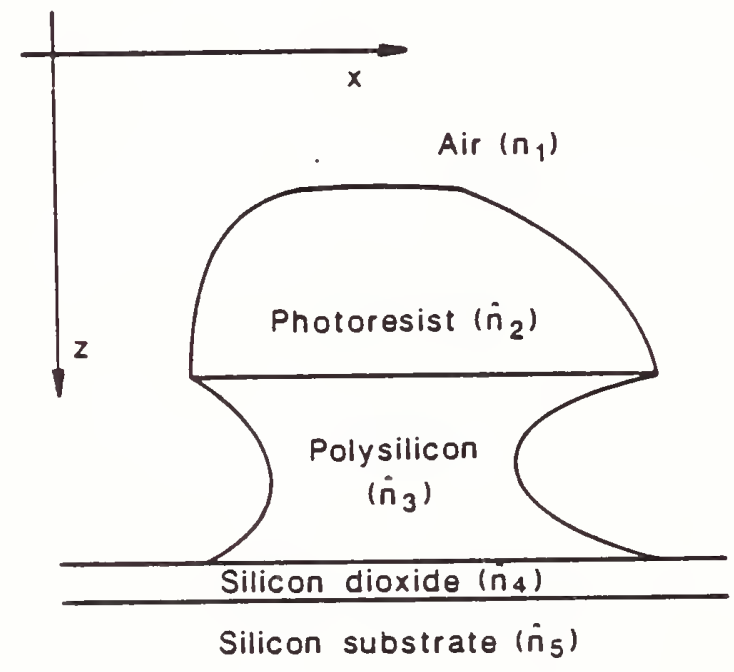

(a)

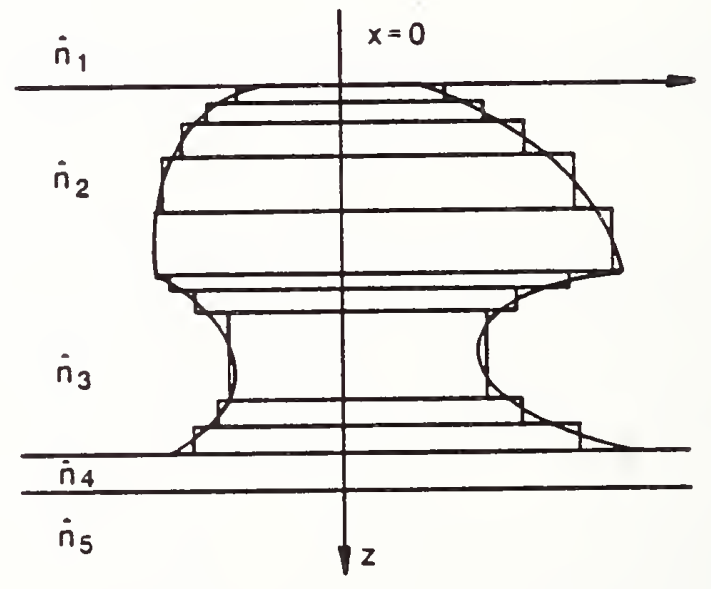

(b)

Fig. 2. Cross section of a hypothetical thick Iine object (a) and the corresponding multi-layer representation (b). 


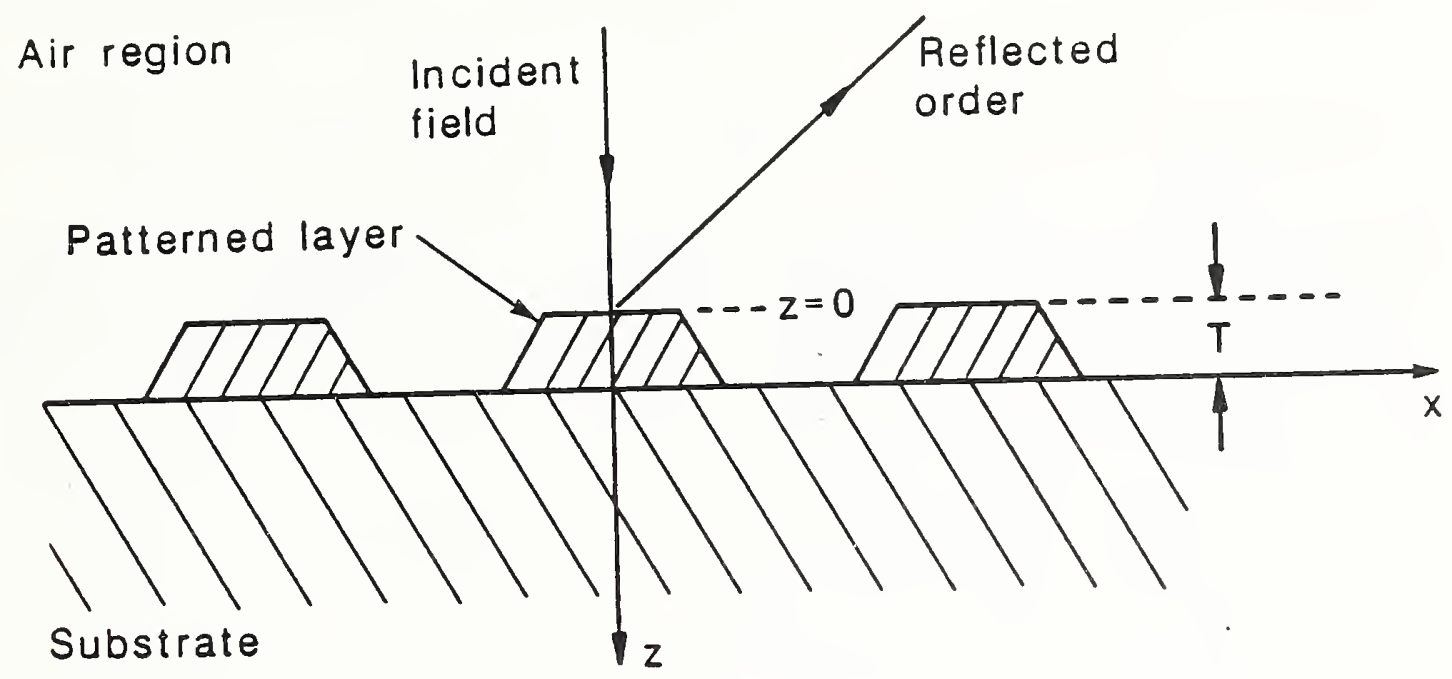

Fig. 3. Orientation of the line structure and incident fields in the NBS linewidth measuring system. The $y$-direction is out of the page and parallel to the length of the line patterns.

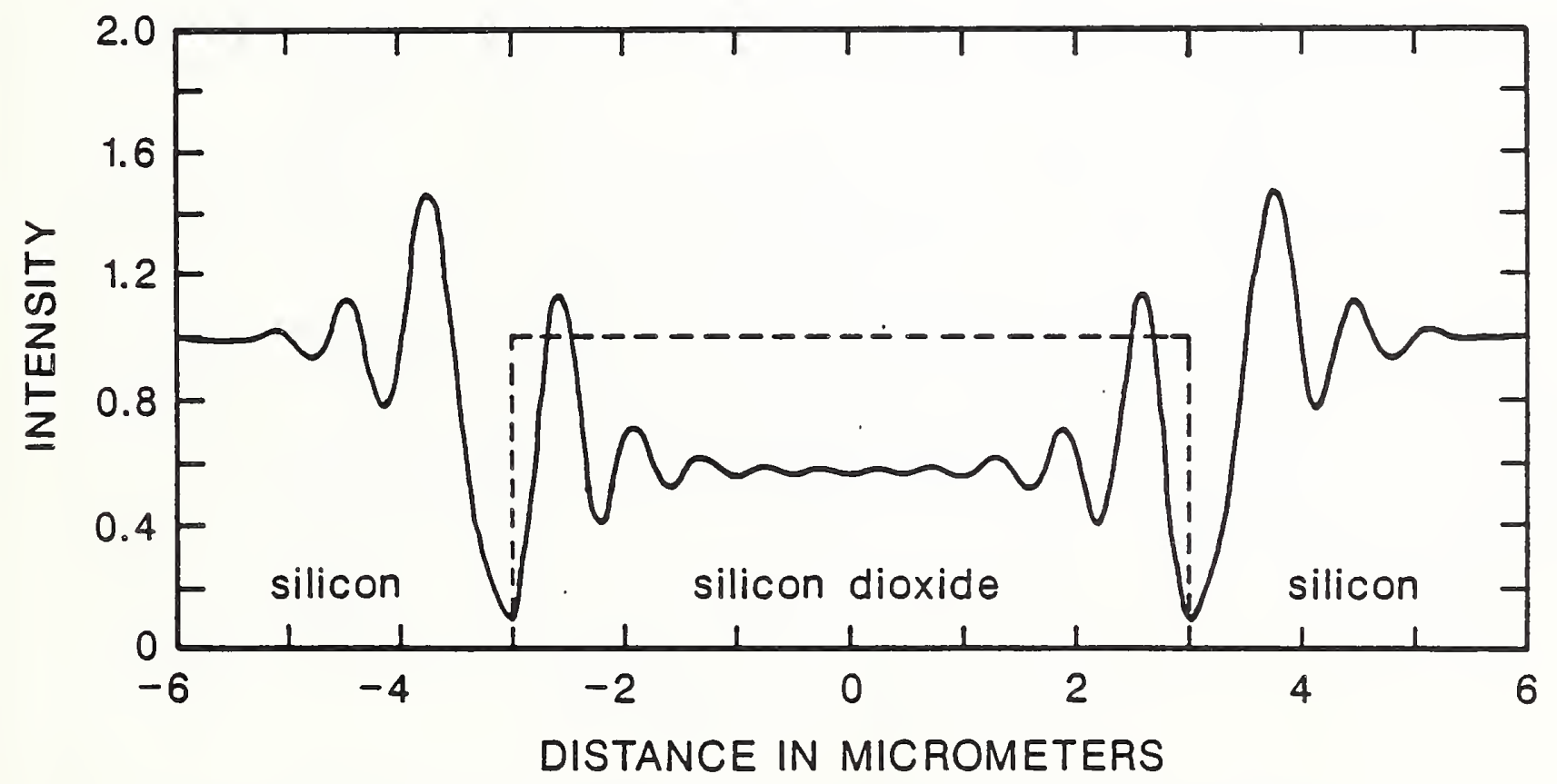

Fig. 4. Theoretical image intensity profile of a $6.0 \mu \mathrm{m}$ wide line centered at zero, patterned in a $0.6 \mu \mathrm{m}$ thick $\mathrm{sio}_{2}$ layer on sili-
con. $\lambda=530 \mathrm{~nm}$. 
First Order

$W(z)=6.0+X_{1} \cdot z$
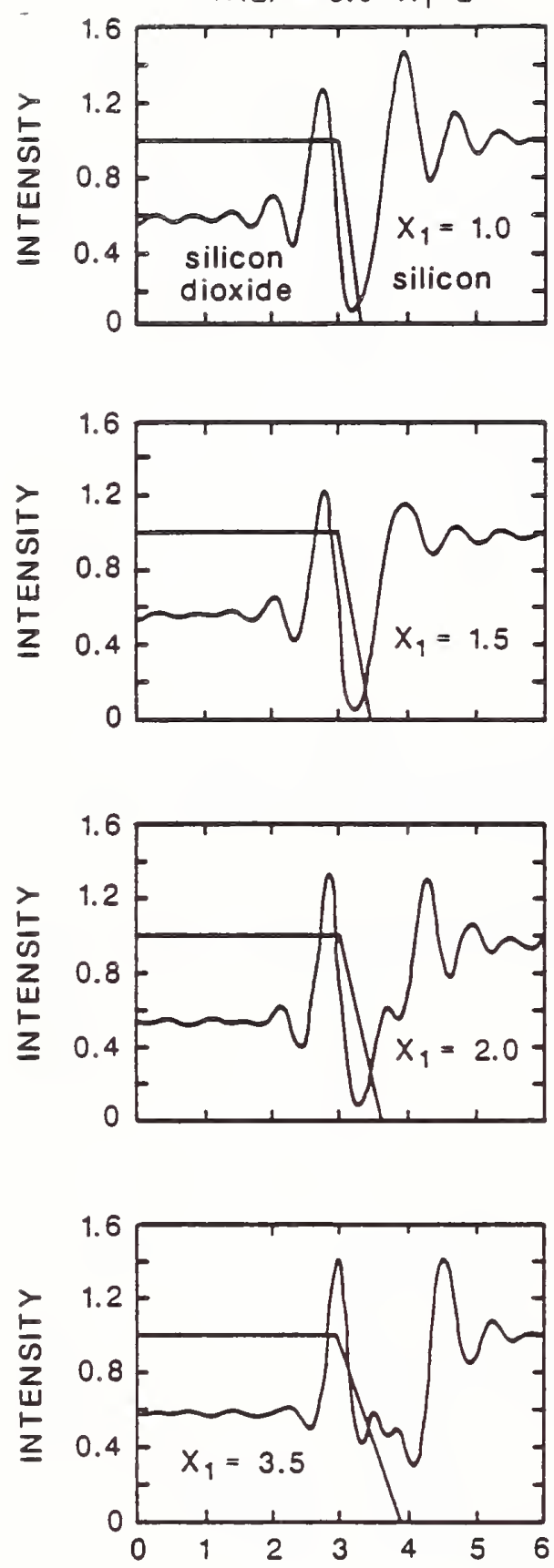

Second Order

$W(z)=6.0+X_{2}(z-0.6)^{2}$

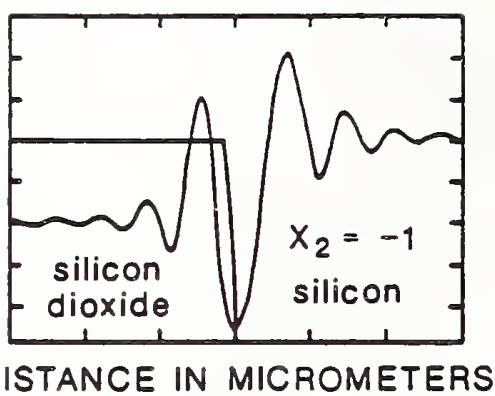

DISTANCE IN MICROMETERS

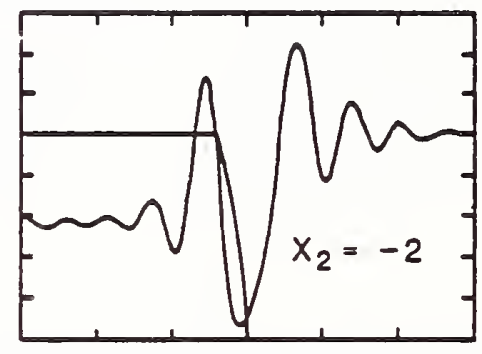

DISTANCE IN MICROMETERS

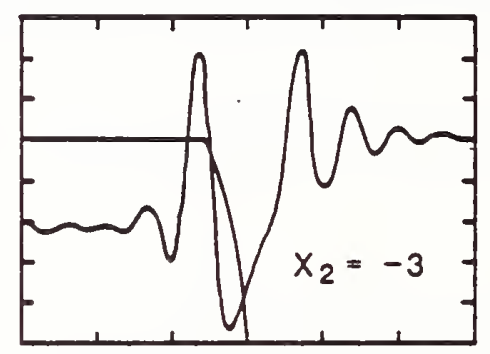

DISTANCE IN MICROMETERS
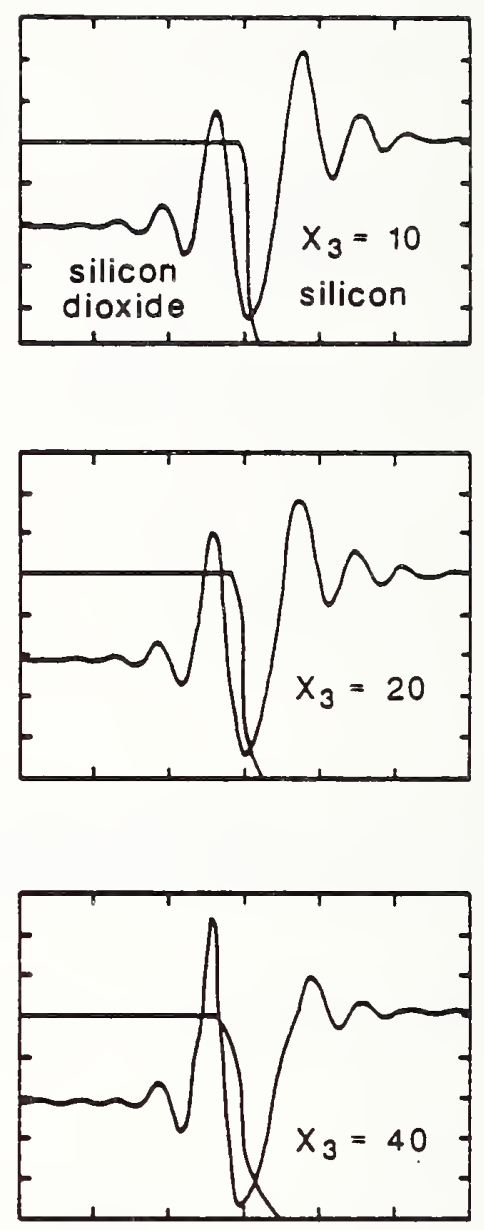

$W(z)=6.0+X_{3}(z-0.3)^{3}$
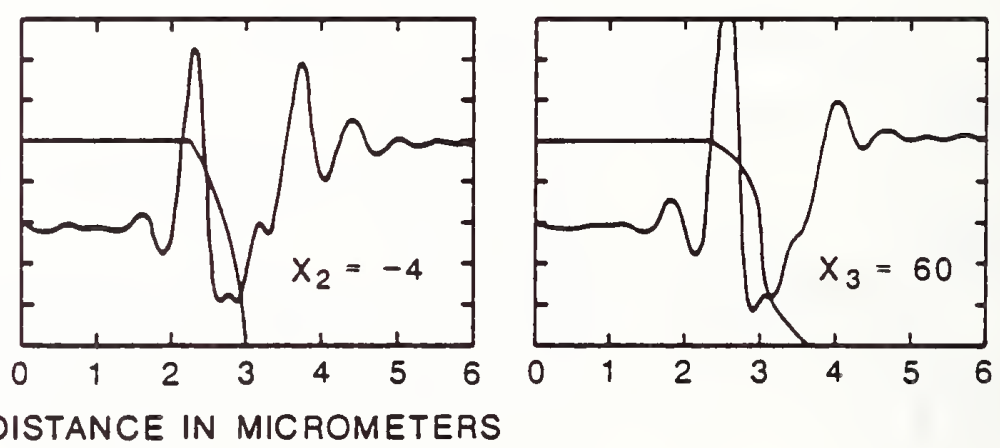

Fig. 5. The effect of edge geometry on the calculated optical image profile. The edge geometry has been superimposed on the image profile for reference. 

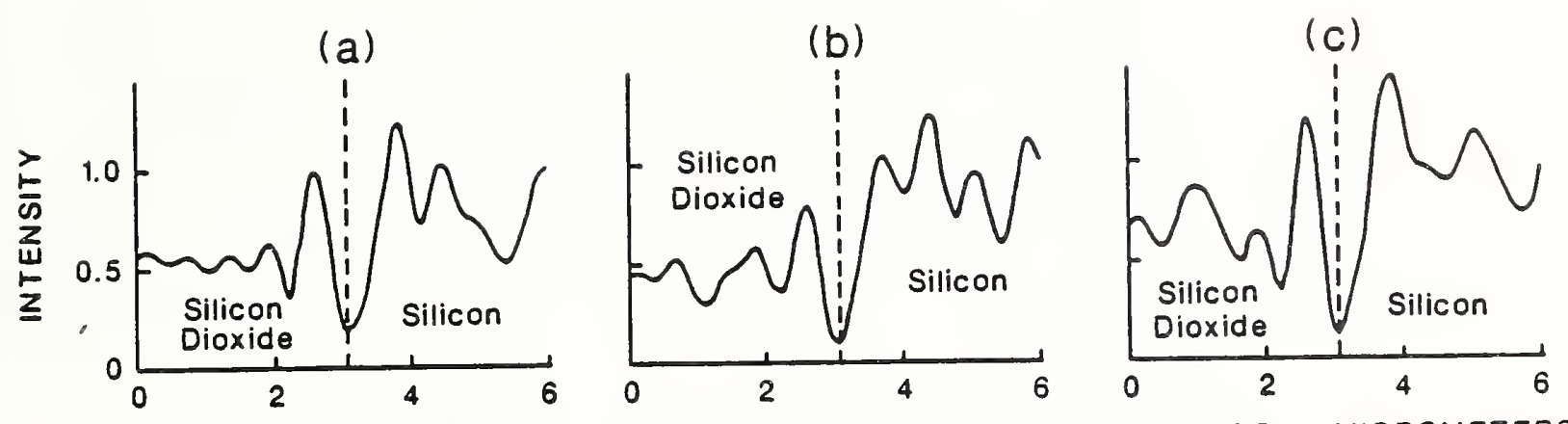

DISTANCE IN MICROMETERS DISTANCE IN MICROMETERS DISTANCE IN MICROMETERS

Fig. 6. Theoretical image intensity profiles of a line patterned in a noisy dielectric layer on silicon. A different set of noise data has been used for each curve.

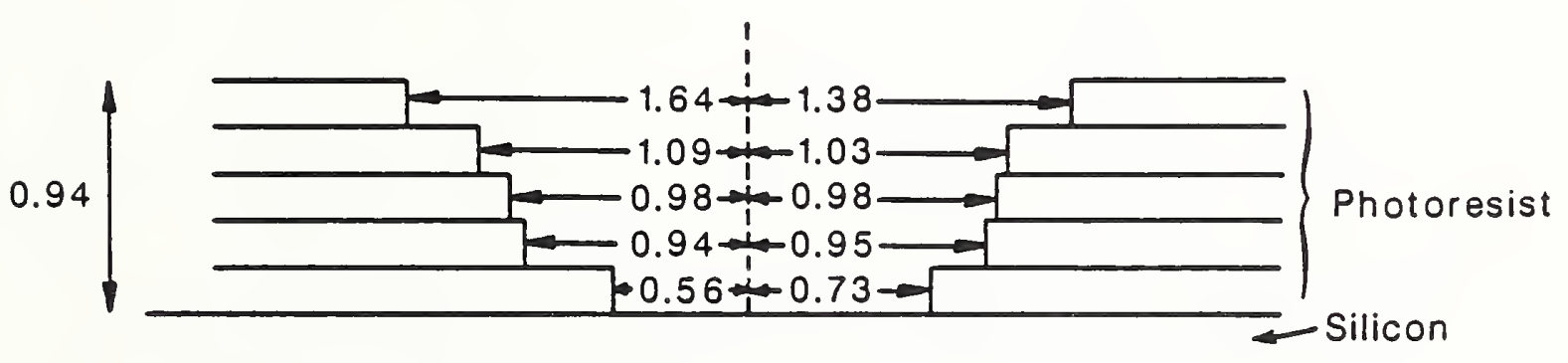

Fig. 7. Physical profile model of the asymmetric window structure in resist used to compute the image profiles in Fig. 8. (Dimensions in micrometers.) 

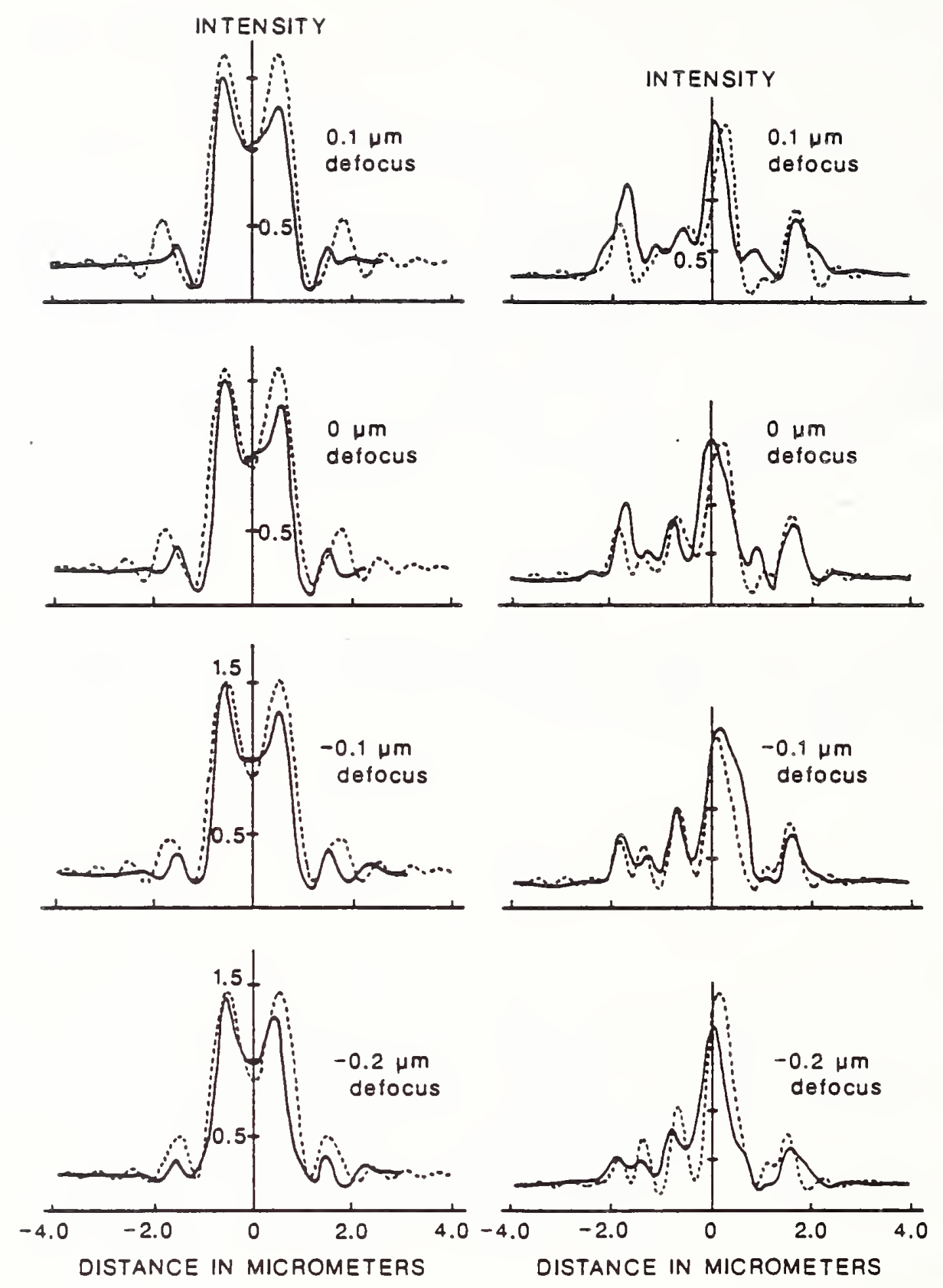

Fig. 8. Comparison of experimental and theoretical image intensity profiles of a window in photoresist on silicon. The window has either vertical edge walls (a) or sloping edges (b). The experimental profiles are shown by the thick line and the theoretical profiles by the thin line. The photoresist was assumed to have a thickness of $0.94 \mathrm{\mu m}$ and the geometry shown in Fig. 7 was used to model the window in the case of sloping edges. 


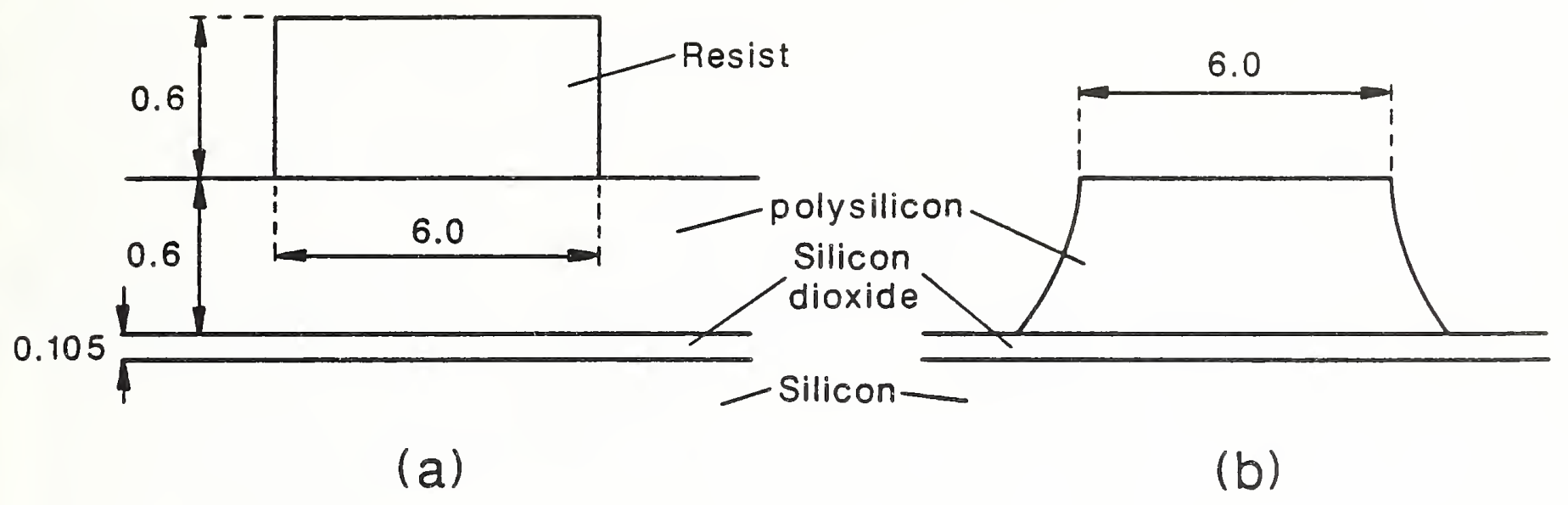

Fig. 9. Cross sections of the shapes used to model the polysilicon patterning stage of making an Mos transistor. The two shapes are patterned resist in unetched polysilicon (a) and etched polysilicon with the resist removed (b). (Dimensions in micrometers.)
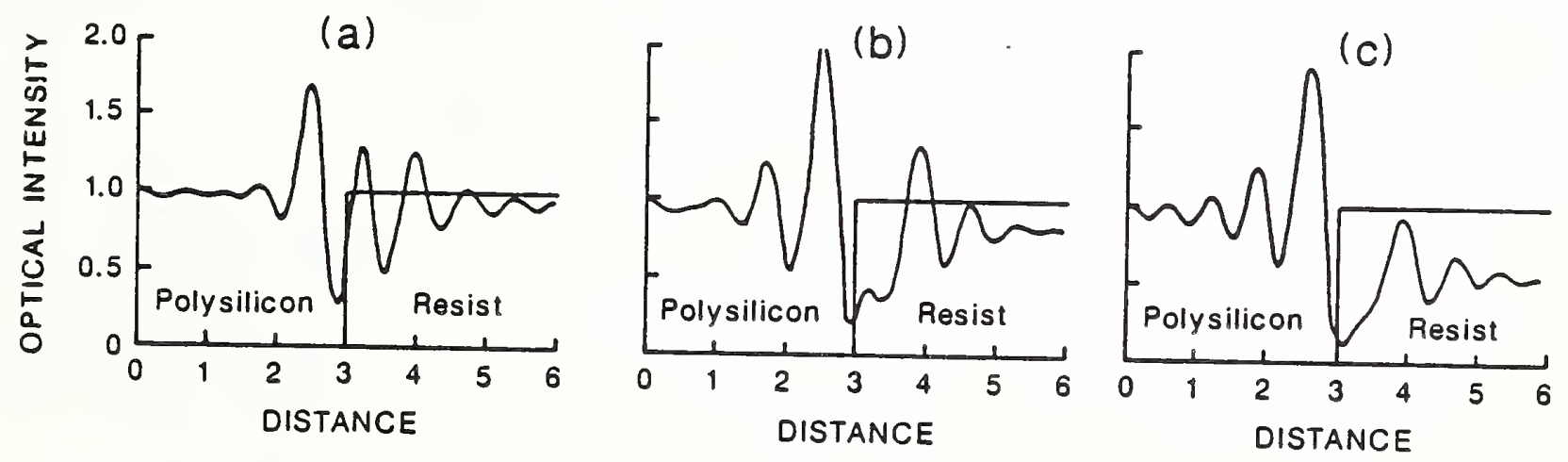

Fig. 10. Theoretical image profiles of a resist line on polysilicon (structure in Fig. 9a) for polysilicon thicknesses of a) 0.5 , b) 0.6 , and c) $0.7 \mu \mathrm{m}$. A vertical edge is assumed. 

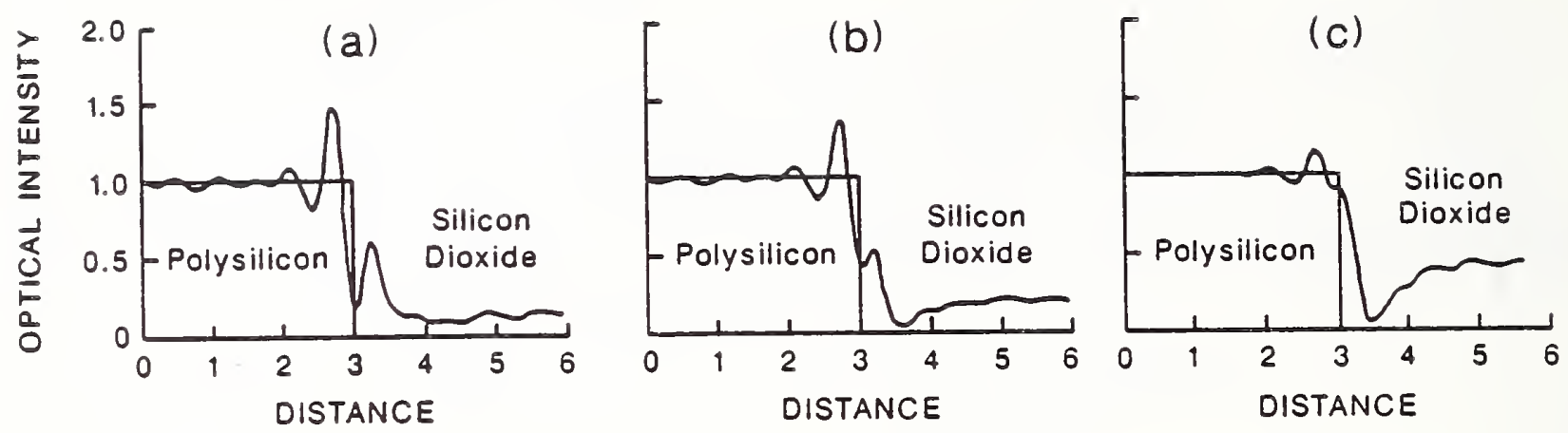

Fig. 11. Theoretical image profiles of a polysilicon line (structure in Fig. 9b) for oxide thicknesses of a) 85, b) 105, and c) $125 \mathrm{~nm}$. A vertical edge is assumed and $0.6 \mu \mathrm{m}$ polysilicon thickness.
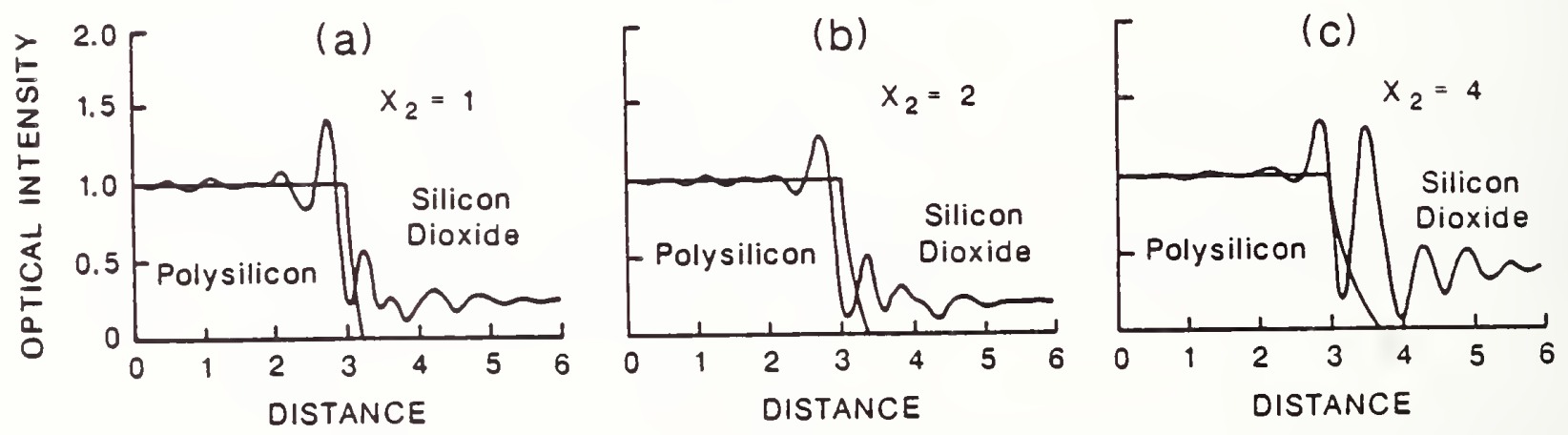

Fig. 12. Theoretical image profiles of a polysilicon line (structure in Fig. 9b) for a range of edge curvatures defined by Eq. 26. oxide thickness is $105 \mathrm{~nm}$; polysilicon thickness is $0.6 \mu \mathrm{m}$. 


\section{FEDERAL INFORMATION PROCESSING STANDARD SOFTWARE SUMMARY}

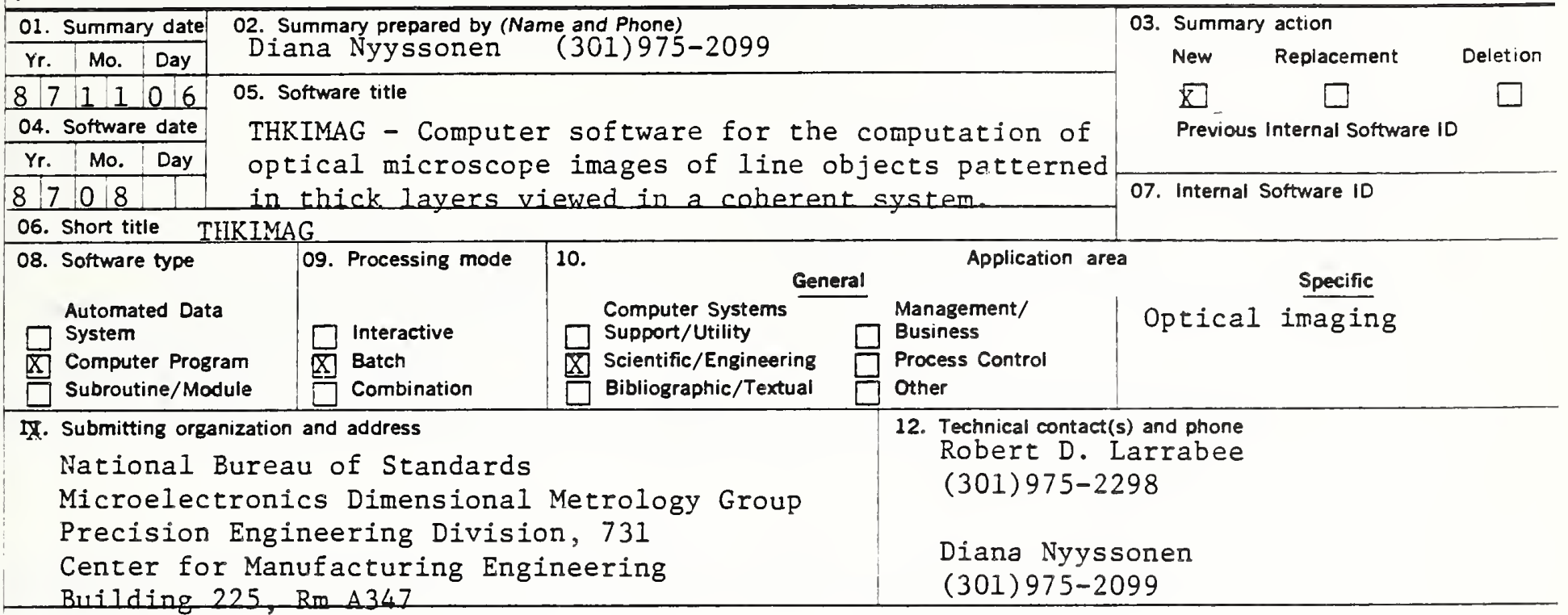

13. Narrative

This computer software calculates the optical microscope images of line objects patterned in thick layers (more than one-quarter of the illuminating wavelength thick). The algorithms used are based on a monochromatic, waveguide model which can predict the image of line objects with arbitrary cross-section geometry including multilayer structures with sloped, curved, asymmetric, and undercut edges. Along with the computer software, test cases for help in implementation are given. As written, the program uses subroutines for complex matrix elgenvalues and eigenvectors from the NAG library.

14. Keywords

diffraction; dimensional metrology; linewidth; microscopy; optical imaging; optical metrology; waveguide

\begin{tabular}{|c|c|c|c|}
\hline $\begin{array}{l}\text { 15. Computer manuf'r and model } \\
\text { Cyber } 855 \text { (CDC) }\end{array}$ & $\begin{array}{l}\text { 16. Computer operating system } \\
\text { NOS } 2 \text { (CDC) }\end{array}$ & $\begin{array}{l}\text { 17. Programing language(s) } \\
\text { ANSI FORTRAN77 }\end{array}$ & $\begin{array}{l}\text { 18. Number of source program state- } \\
\text { ments } \\
949\end{array}$ \\
\hline $\begin{array}{l}\text { 19. Computer memory requirements } \\
91170 \text { words. }\end{array}$ & 20. Tape drives & $\begin{array}{l}\text { 21. Disk/Drum units } \\
120 \mathrm{~K} \text { words }\end{array}$ & 22. Terminals \\
\hline
\end{tabular}

23. Other operational requirements

\begin{tabular}{ll|l|l|l|}
\hline 24. Software availability \\
Available
\end{tabular}

26. FOR SUBMITTING ORGANIZATION USE 
NBS-114A (REV. 2.8C)

U.S. DEPT. OF COMM.

BIBLIOGRAPHIC DATA

SHEET (See in structions)

1. PUBLICATION OR REPORT NO.

NBSIR $87 \quad 3618$

4. TITLE AND SUBTITLE

Computer Software for the Computation of the Scattered Field and the Optical Microscope Image of Line Objects Patterned in Thick Layers

5. $\operatorname{AUTHOR}(S)$

6. PERFORMING ORGANIZATION (If joint or other than NBS, see instructions)

7. Contracd Grant No.

NATIONAL BUREAU OF STANDARDS

U.S. DEPARTMENT OF COMMERCE

GATTHERSBURG, MD 20899

9. SPONSORING ORGANIZATION NAME AND COMPLETE ADDRESS (Street, City, State, ZIP)

8. Type of Report \& Period Covered

10. SUPPLEMENTARY NOTES

Document describes a computer program; SF-185, FIPS Software Summary, is attached.

11. ABSTRACT (A 200-word or less factual summory of most significant information. If tocument includes a significant bibliography or literature survey. mention it here)

This report contains computer software for calculating optical microscope imaces of Iine objects patterned in thick layers ( $>\lambda / 4$ thick). The algorithms used are based on a monochromatic, waveguide model which can predict the images of line objects with arbitrary edge geometry including multilayer structures with sloped, curved, asymmetric, and undercut edges. Along with the computer software listing, the mathematics of the model, a short description of its structure and use, and test cases for help in implementation are given.

12. KEY WORDS (Six to iwelve entries; alphabetical order; capitalize only proper names; and separaie key words by semicolons) diffraction; dimensional metrology; linewidth; microscopy; optical imaging; optical metrology; computer software

13. AVAILABILITY

Unlimited

For Official Distribution. Do Not Release to NTIS

Order From Superintendent of Documents, U.S. Government Printing Office, Washington, D.C. 20402.

※ Order From National Technical Information Service (NTIS), Springfield, VA. 2216I
14. NO. OF PRINTED PAGES

86

15. Price

$\$ 13.95$ 
$-$ 
